\title{
Fairness in hazmat routing-scheduling: a bi-objective Stackelberg game
}

Article

Accepted Version

Creative Commons: Attribution-Noncommercial-No Derivative Works 4.0

Mohri, S., Asgari, N., Farahini, R. Z., Bourlakis, M. and Laker, B. (2020) Fairness in hazmat routing-scheduling: a bi-objective Stackelberg game. Transportation Research Part E: Logistics and Transportation Review, 140. 102006. ISSN 1366-5545 doi: https://doi.org/10.1016/j.tre.2020.102006 Available at https://centaur.reading.ac.uk/91125/

It is advisable to refer to the publisher's version if you intend to cite from the work. See Guidance on citing.

To link to this article DOI: http://dx.doi.org/10.1016/j.tre.2020.102006

Publisher: Elsevier

All outputs in CentAUR are protected by Intellectual Property Rights law, including copyright law. Copyright and IPR is retained by the creators or other copyright holders. Terms and conditions for use of this material are defined in the End User Agreement.

\section{www.reading.ac.uk/centaur}

\section{CentAUR}

Central Archive at the University of Reading 
Reading's research outputs online 


\section{Title page}

Sina Mohri (MSc)

Department of Transportation Engineering, Isfahan University of Technology, Iran ss.mohri@gmail.com

\section{Nasrin Asgari $(P h D)$}

Roehampton Business School, University of Roehampton, London, United Kingdom nasrin.asgari@roehampton.ac.uk

Reza Zanjirani Farahani (PhD) (corresponding author)

* Associate Professor, Department of Management, Kingston Business School KINGSTON BUSINESS SCHOOL

Room KHBS-2015, Department of Management, Kingston Business School, Kingston University, Kingston Hill, Kingston Upon Thames, Surrey, The UK, KT2 7LB

T: $+44(0) 2084175098$ |F: + 44 (0)20 84177026

E: R.ZanjiraniFarahani@kingston.ac.uk; Zanjiranireza@ gmail.com

\section{Michael Bourlakis (PhD)}

Cranfield School of Management, Cranfield University, United Kingdom m.bourlakis@cranfield.ac.uk

\section{Benjamin Laker (PhD)}

Henley Business School, The University of Reading, United Kingdom benjamin.laker@henley.ac.uk 


\title{
Fairness in Hazmat Routing-Scheduling: A Bi-Objective Stackelberg Game
}

\begin{abstract}
We investigate a hazmat routing-scheduling problem. To minimize the overall expected risk, various vehicles may take different routes/schedules to avoid multiple accidents on the same link. Therefore, the company envisages two issues: (1) unfairly, a vehicle departing earlier from its origin may arrive earlier at its destination than the others leaving later; (2) focusing only on the minimization of risk may increase travel time/cost incurred by the company. We suggest a bi-objective game-theoretic formulation and solve it by a modified Adaptive Large Neighborhood Search and Simulated Annealing. We test the solution on a real-life case and extract practical insights.
\end{abstract}

Keywords: Transportation; Vehicle routing; Hazardous materials; Game theory; Multi-objective decision making. 


\section{INTRODUCTION}

Hazmat (hazardous material) refers to any type of substance that can be harmful to people's lives, the infrastructure, or the environment. Hazmats can be categorized into explosive gases, flammable and combustible liquids, flammable solids, oxidizers and organic peroxides, toxic materials, infectious substances, radioactive materials, corrosive materials and miscellaneous dangerous goods. The vast majority of domestic hazmat shipments are related to flammable liquids -which is the focus of this research-and may reach up to $85 \%$ of all hazmat shipments by weight (Bureau of Transportation Statistics, 2017b). The statistics show the volume of hazmat shipments has been increasing in the world. For instance, in 2012, 2015, and 2018, the total shipment of crude oil and petroleum products in the US was nearly 3.1, 3.9, and 4.5 million tons, respectively (US Department of Energy, 2019). The tonnage of hazmat shipment in the US is estimated to increase at the rate of 1.4 percent per year between 2015 and 2045 (Bureau of Transportation Statistics, 2017a). Nearly $15 \%$ of the tonnage and $13 \%$ of the value of total freight consists of hazardous materials in the US (Bureau of Transportation Statistics, 2017a).

Similar to normal materials, hazmats are shipped through various transportation modes (such as land, water, air and pipes). Trucks moved 59.4 percent of the tonnage and 62.8 percent of the value of all the hazardous materials shipped from within the US (Bureau of Transportation Statistics, 2017a). In the hazmat context, the land transportation mode -what is studied in our research-is more challenging than the other modes because it may pass through residential areas and endanger people's lives (Romero et al., 2016; Bronfman et al., 2016). In 2017, the number of hazmat incidents on US highways alone was 15,311, causing 123 injuries (hospitalized and nonhospitalized), six fatalities and \$40,548,785 worth of damage (PHMSA, 2018). In this research, hazmat transportation planning with a special focus on road routing-scheduling by trucks is investigated. Observing fairness among the trucks and investigating trade-offs between risk and travel time are the core values of the research.

Motivation: To mitigate risk in hazmat land transportation, two approaches are usually taken by shipment companies: 1) routing, which geographically separates the routes of hazmat shipped simultaneously, and 2) scheduling, which separates the transportation time slots of hazmat shipments on the same route. Routing and scheduling can be applied either sequentially or concurrently, aiming at minimizing the overall risk in hazmat transportation. In this research, we consider both approaches simultaneously (i.e. routing-scheduling). It should be noted that the hazmat routing problem is divided into three decision categories (Holeczek, 2019): (i) Shortest path problems (SPP), (ii) dissimilar path problems (DPP), and (iii) vehicle routing problems (VRP). According to this classification, our research belongs to SPP, which is also integrated with a scheduling problem.

While applying sequential decision-making needs less computational effort, concurrent planning usually leads to improved solutions in terms of objectives and performance measures such as risk (i.e. the number of casualties and fatalities) and travel time/ cost. This research focuses on simultaneous routing-scheduling decision making for land, hazmat transportation in urban/ residential areas. In the existing literature, the abovementioned two approaches do not guarantee that trucks departing earlier reach their destination earlier; this is not fair to the dispatchers (e.g., truck drivers) as they are paid in terms of ton-kilometers, not working hours. In this research, we enforce the FirstIn-First-Out (FIFO) discipline to establish fairness. However, this makes the problem mathematically complicated. Scholars including Larson (1987), Gopalan et al. (1990), Schmitt et al. (1992) and Zhou and Soman (2008) explored the role of FIFO as a proxy for social justice and fairness that will be explained in the literature review.

First, in highly populated urban areas such as Singapore and Hong Kong, hazmat truck drivers are not supposed to take the same routes. In other words, it is high-risk that trucks move independently while easily maintaining safe distances. For example, there are several long underground tunnels in populated islands like Singapore and Hong 
Kong. Any possible hazmat explosion within any of these tunnels, which are on an approved route, disrupts it for a long time. Therefore, in case of any advance incident, the following trucks on the route that move with a headway (identified distance) cannot pass. Therefore, trucks need to be scheduled apart on different routes or through different routes. Since there is not sufficient historical information (i.e. sufficient number of incidents) on all links to calculate the probability of incidents for all of them, we apply the concept of imaginary demons to formulate the problem. Therefore, (1) the demons have intelligence and (2) attack to cause maximum possible damage (i.e. worst-case scenario). Since the worst-case scenario is only applied to extremely risk-averse decision-makers, we have considered a second objective function which is economic. By considering different weights on risk and travel time/ cost, we can embed the risk attitude of the decision-maker so that it is not just limited to risk-averse decision-makers.

Second, practically, in freight transportation organizations, cargo payments are based on travel distance, not travel time. If the trucks moving between the same Origin-Destination (OD) pairs take different routes, they will spend different travel times while this will not be included in their payments. This will be psychologically perceived as a lack of fairness by drivers and a managerial issue by the transportation company. They will decide not to wait on these places. Moreover, it is tough work for a manager to force the drivers to stop in waiting places that do not have a fair queuing system. Therefore, managers may exclude the option of waiting in a route that leads to ruining one of its objectives (e.g., minimization travel time or minimization of risk).

Another motivation of the research is regarding uncertainty in incidents. Hazmat incidents rarely happen and usually, there is not sufficient historical data to quantify the probability of incidents for "all" road links (note that we may have sufficient historical data only for some-not all-links). The vast majority of papers in the literature consider known incident probability and only recently few papers -like this research- have realistically studied unknown probability. Note that hazmat routing-scheduling decisions are dealing with low-probability-high-consequence events. To calculate incident probabilities, sufficient historical data is required for "all" links. Since the number of hazmat incidents in every link is statistically insufficient, the incidents probabilities are unknown for many links. Considering unknown probabilities makes scholars exploit a risk-based objective function for a worst-case scenario which is appropriate for a risk-averse decision-maker looking only at a social security-based objective function. In this research, we also add a time/cost-based objective function to create a model for decision-makers with different risk attitudes. Therefore, risk-neural and risk-seeking decision makers may partially or totally consider an economic objective function (e.g., minimization of travel time, distance or cost). Therefore, the research problem investigated in this study is bi-objective in nature, considering both social and economic aspects. Consequently, there will be flexibility in decision making to adjust solutions based on the risk attitude of decision makers.

Another motivation for this research is related to its modeling and solution concept. Considering unknown incident probabilities and, consequently, a worst-case scenario solution- prior to including the time/cost objectiveresembles intentional attacks of imaginary demons who plot to attack so that to cause the most significant possible incident damage cause hazmat incidents. Hence, to plan for the worst-case scenario, the proposed hazmat routingscheduling problem is considered as a game between the transportation company and the imaginary demons. In reality, it is a leader-follower game so that the transportation planner makes the first move (by making routingscheduling decisions). Then the demons decide to target the shipments accordingly to cause the highest possible damage. Hence, while the research problem is bi-objective (including risk and time objectives) for a transportation company, at the same time, it is modeled and solved as a Stackelberg game (unlike Szeto et al. (2017 that consider it a Nash game) played between the transportation company and demons over the risk objective.

Contribution: In this research, we have designed a modified metaheuristic algorithm to solve the research 
model efficiently. This research contributes to the literature in the following ways: (1) applying the FIFO discipline to the movement of dispatchers as a matter of fairness in practice; (2) formulating a link-based, demon approach with two objective functions based on a Stackelberg game; and (3) developing a modified metaheuristic method based on Adaptive Large Neighborhood Search (ALNS) and Simulated Annealing (SA) to tackle the problem. The modifications applied to ALNS and SA improve the process of selecting the nominated removal/ insertion moves and investigating the acceptance criterion. Finally, yet importantly, we have applied the validated model on a realistic data set. We perform some sensitivity analysis on the model to provide rudimentary but exciting and useful insights for practitioners.

Organization: The organization of the paper is as follows: The related literature review and the academic contribution of the research are presented in section 2. The problem is described in detail in section 3 and formulated in section 4. Our designed solution approach is presented in section 5. In section 6, parameter tuning is performed and the model is validated based on some test problems of various sizes. In section 7, the road network of Singapore is presented. Some sensitivity analyses are done in section 8, and insights are presented. Finally, the paper is concluded in section 9 .

\section{LITERATURE REVIEW}

Safety and security in transportation systems, including hazmat movements, have already been studied from various perspectives. Enhancing and integrating technology (Huang et al., 2004; DeLorenzo et al., 2005), regulating rules (Gordon and Young, 2015) and optimizing routing- and scheduling decisions are various approaches that may improve transportation safety and security. Hazmat routing and routing-scheduling problems have a fairly rich literature (refer to Huang et al., 2004; Bell, 2006; Carotenuto et al., 2007a; Bersani et al., 2010; Kazantzi et al., 2011; Li and Leung, 2011; Xiang et al., 2012; Li et al., 2013; Kang et al., 2014; Mahmoudabadi and Seyedhosseini, 2014; Mishra et al., 2014; Rahman et al., 2014; Pamučar et al., 2016; Sahnoon et al., 2016; Sun et al., 2016; Bula et al., 2017; Özceylan et al., 2017; Wang et al., 2017; Yuan et al., 2017; Sun et al., 2018). Since the focus of this research is on hazmat routing-scheduling, in the upcoming subsections we only focus on hazmat routing-scheduling research studies and exclude pure hazmat routing research studies. To learn the literature of the genuine routing problem and its derivatives (e.g. hazmat locating-routing, and transportation network design), interested readers may refer to the latest review paper published by Holeczek (2019). In subsection 2.1, we critically compare and contrast the main features of our research against the existing literature. The contributions of the research are summarized in subsection 2.2 .

\subsection{Positioning the Research in the Literature}

In this subsection, we discuss the features of the problems investigated in the hazmat routing-scheduling literature. Table 1 demonstrates the investigated features of hazmat routing-scheduling papers in the literature. We compare and contrast the main features of our research problem against the existing papers in terms of objective, constraint, assumption (e.g., fleet size, hazmat classes, and vehicle types), complexity (e.g., link-based or route-based formulations) and solution method. In the last row of the table, this research is compared and contrasted with the literature papers. 
Table 1: Features of hazmat routing-scheduling papers in the literature.

\begin{tabular}{|c|c|c|c|c|c|c|c|c|c|c|c|}
\hline Reference & 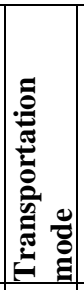 & The objectives for decision makers & 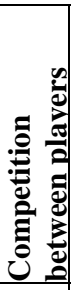 & 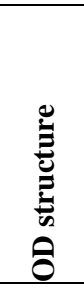 & 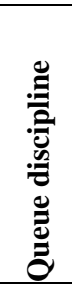 & 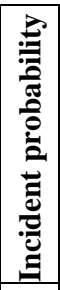 & 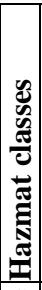 & & 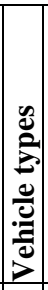 & 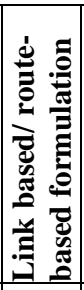 & 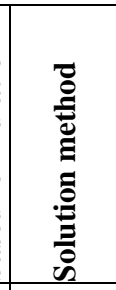 \\
\hline Nozick et al. (1997) & RO I & Dispatcher: Min [Population exposure \& rate of incident] & - & SO & $\mathrm{N} / \mathrm{N}$ & $\mathrm{K}$ & $\mathrm{S}$ & $\mathrm{N}$ & $\mathrm{S}$ & $\mathrm{RB}$ & $\mathrm{E}$ \\
\hline Haghani and Chen (2003) & RO & Dispatcher: Min [Total travel time \& expected risk] & - & $\mathrm{SO}$ & $\mathrm{N} / \mathrm{N}$ & $\mathrm{K}$ & $\mathrm{S}$ & $\mathrm{N}$ & $\mathrm{S}$ & LB & $\mathrm{E} \& \mathrm{H}$ \\
\hline $\begin{array}{l}\text { Zografos and } \\
\text { Androutsopoulos (2004) }\end{array}$ & RO II & Dispatcher: Min [Total travel time \& expected risk] & - & DC & $\mathrm{N} / \mathrm{N}$ & $\mathrm{K}$ & $\mathrm{S}$ & $\mathrm{Y}$ & $\mathrm{M}$ & LB & $\mathrm{H}$ \\
\hline Meng et al. (2005) & RO & $\begin{array}{l}\text { Dispatcher: Min [Total travel time \& population } \\
\text { exposure \& rate of incident] }\end{array}$ & - & SO & $\mathrm{Y} / \mathrm{N}$ & $\mathrm{K}$ & $\mathrm{S}$ & $\mathrm{N}$ & $\mathrm{S}$ & LB & $\mathrm{H}$ \\
\hline Chang et al. (2005) & RO & $\begin{array}{l}\text { Dispatcher: Min [Total travel time \& incident probability } \\
\& \text { population exposure] }\end{array}$ & - & SO & $\mathrm{N} / \mathrm{N}$ & K & $\mathrm{S}$ & $\mathrm{N}$ & $\mathrm{S}$ & RB & $\mathrm{H}$ \\
\hline Akgün et al. (2007) & RO I & Dispatcher: Min [Expected risk] & - & MO & $\mathrm{Y} / \mathrm{N}$ & $\mathrm{K}$ & $\mathrm{S}$ & $\mathrm{N}$ & $\mathrm{S}$ & $\mathrm{RB}$ & $\mathrm{E}$ \\
\hline Carotenuto et al. (2007) & RO & $\begin{array}{l}\text { Dispatcher: Min [Total shipment delay \& general } \\
\text { measure of risk] }\end{array}$ & - & MO & $\mathrm{N} / \mathrm{N}$ & $\mathrm{K}$ & $\mathrm{S}$ & $\mathrm{N}$ & $\mathrm{S}$ & RB & $\begin{array}{l}\mathrm{MH} \\
(\mathrm{TS})\end{array}$ \\
\hline Erkut and Alp (2007) & RO & Dispatcher: Min [Expected risk] & - & $\mathrm{SO}$ & $\mathrm{Y} / \mathrm{N}$ & $\mathrm{K}$ & $\mathrm{S}$ & $\mathrm{N}$ & $\mathrm{S}$ & LB & $\mathrm{E}$ \\
\hline $\begin{array}{l}\text { Zografos and } \\
\text { Androutsopoulos (2008) }\end{array}$ & RO I & Dispatcher: Min [Total travel time \& expected risk] & - & MO & $\mathrm{N} / \mathrm{N}$ & $\mathrm{K}$ & $\mathrm{M}$ & $\mathrm{N}$ & $\mathrm{M}$ & LB & $\mathrm{H}$ \\
\hline Pradhananga et al. (2009) & RO & $\begin{array}{l}\text { Dispatcher: Min [Total travel time \& expected risk \& } \\
\text { number of vehicles] }\end{array}$ & - & DC & $\mathrm{Y} / \mathrm{N}$ & K & $\mathrm{S}$ & $\mathrm{Y}$ & $\mathrm{M}$ & $\mathrm{RB}$ & $\begin{array}{c}\mathrm{MH} \\
(\mathrm{ACO})\end{array}$ \\
\hline Pradhananga et al. (2010) & RO & $\begin{array}{l}\text { Dispatcher: Min [Total travel time } \& \text { expected risk } \& \\
\text { number of vehicles] }\end{array}$ & - & DC & $\mathrm{Y} / \mathrm{N}$ & K & $\mathrm{S}$ & $\mathrm{Y}$ & $\mathrm{M}$ & $\mathrm{RB}$ & $\begin{array}{c}\mathrm{MH} \\
(\mathrm{ACO})\end{array}$ \\
\hline $\begin{array}{l}\text { Androutsopoulos and } \\
\text { Zografos (2010) }\end{array}$ & RO I & Dispatcher: Min [Total travel time \& perceived risk] & - & $\begin{array}{c}\text { MO\& } \\
\text { DC }\end{array}$ & $\mathrm{Y} / \mathrm{N}$ & $\mathrm{K}$ & $\mathrm{S}$ & $\mathrm{N}$ & $\mathrm{S}$ & RB & $\mathrm{E}$ \\
\hline Ma et al. (2012) & RO & $\begin{array}{l}\text { Dispatcher: Min [Total travel time \& num } \\
\text { vehicles] }\end{array}$ & - & DC & $\mathrm{N} / \mathrm{N}$ & - & $\mathrm{S}$ & $\mathrm{N}$ & $\mathrm{S}$ & LB & $\begin{array}{l}\mathrm{MH} \\
(\mathrm{TS})\end{array}$ \\
\hline \begin{tabular}{|l|} 
Androutsopoulos and \\
Zografos (2012) \\
\end{tabular} & RO I & Dispatcher: Min [Total travel time \& expected risk] & - & SO & $\mathrm{N} / \mathrm{N}$ & K & $\mathrm{S}$ & $\mathrm{Y}$ & $\mathrm{M}$ & LB & $\mathrm{H}$ \\
\hline Hosseinloo et al. (2012) & RO t & $\begin{array}{l}\text { Dispatcher: Min [Total travel time \& expected risk \& } \\
\text { total delay] } \\
\text { Demon: Max [Total expected risk and total delay] }\end{array}$ & $\mathrm{Na}$ & SO & $\mathrm{N} / \mathrm{N}$ & $\mathrm{UN}$ & $\mathrm{S}$ & $\mathrm{N}$ & $\mathrm{S}$ & LB & $\mathrm{H}$ \\
\hline Toumazis and Kwon (2013) & RO & Dispatcher: Min [CVaR] & - & $\mathrm{SO}$ & $\mathrm{N} / \mathrm{N}$ & $\mathrm{K}$ & $\mathrm{S}$ & $\mathrm{N}$ & $\mathrm{S}$ & $\mathrm{RB}$ & $\mathrm{E}$ \\
\hline Szeto (2013) & RO & $\begin{array}{l}\text { Dispatcher: Min [Expected risk] } \\
\text { Demon: Max [Total expected risk] }\end{array}$ & $\mathrm{Na}$ & SO & $\mathrm{N} / \mathrm{N}$ & UN & $\mathrm{S}$ & $\mathrm{N}$ & $\mathrm{S}$ & LB & $\mathrm{H}$ \\
\hline Pradhananga et al. (2014a) & RO & Dispatcher: Min [Total travel time \& expected risk] & - & $\mathrm{DC}$ & $\mathrm{N} / \mathrm{N}$ & K & $\mathrm{S}$ & $\mathrm{N}$ & $\mathrm{M}$ & LB & $\begin{array}{c}\mathrm{MH} \\
(\mathrm{ACO})\end{array}$ \\
\hline Pradhananga et al. (2014b) & RO & $\begin{array}{l}\text { Dispatcher: Min [Total travel time \& expecte } \\
\text { environmental costs] }\end{array}$ & - & DC & $\mathrm{N} / \mathrm{N}$ & $\mathrm{K}$ & $\mathrm{S}$ & $\mathrm{N}$ & $\mathrm{M}$ & LB & $\begin{array}{c}\mathrm{MH} \\
(\mathrm{ACO})\end{array}$ \\
\hline Siddiqui and Verma (2015) & RO I & Dispatcher: Min [Total monetary cost \& expected risk] & - & MO & $\mathrm{Y} / \mathrm{N}$ & $\mathrm{K}$ & $\mathrm{M}$ & $\mathrm{N}$ & $\mathrm{S}$ & RB & $\mathrm{E}$ \\
\hline Faghih-Roohi et al. (2016) & MA & Dispatcher: Min [CVaR] & - & $\mathrm{DC}$ & $\mathrm{N} / \mathrm{N}$ & $\mathrm{K}$ & $\mathrm{S}$ & $\mathrm{Y}$ & $\mathrm{M}$ & $\mathrm{RB}$ & $\mathrm{E}$ \\
\hline Pradhananga et al. (2016) & RO & $\begin{array}{l}\text { Dispatcher: Min [Total population } \\
\text { congestion cost] }\end{array}$ & - & $\mathrm{DC}$ & $\mathrm{N} / \mathrm{N}$ & - & $\mathrm{S}$ & $\mathrm{N}$ & $\mathrm{M}$ & LB & $\begin{array}{c}\mathrm{MH} \\
(\mathrm{ACO})\end{array}$ \\
\hline Szeto et al. (2017) & RO & $\begin{array}{l}\text { Dispatcher: Min [Expected risk] } \\
\text { Demon: Max [Expected risk] }\end{array}$ & $\mathrm{Na}$ & MO & $\mathrm{Y} / \mathrm{N}$ & $\mathrm{UN}$ & M & $\mathrm{N}$ & $\mathrm{S}$ & LB & $\mathrm{H}$ \\
\hline Fang et al. (2017) & RA & $\begin{array}{l}\text { Dispatcher: Min [General cost of earliness, tardiness and } \\
\text { holding costs] }\end{array}$ & - & MO & $\mathrm{N} / \mathrm{N}$ & - & $\mathrm{S}$ & $\mathrm{N}$ & $\mathrm{M}$ & $\mathrm{RB}$ & $\mathrm{H}$ \\
\hline Chai et al. (2017) & RO I & Dispatcher: Min [Total monetary cost \& risk] & - & MO & $\mathrm{N} / \mathrm{N}$ & - & $\mathrm{S}$ & $\mathrm{N}$ & $\mathrm{S}$ & LB & $\mathrm{MH}(\mathrm{G})$ \\
\hline Hsu et al. (2018) & RO & Dispatcher: Min [Total travel cost] & - & $\mathrm{DC}$ & $\mathrm{N} / \mathrm{N}$ & - & $\mathrm{S}$ & $\mathrm{N}$ & $\mathrm{S}$ & LB & $\mathrm{E}$ \\
\hline Abuobidalla et al. (2019) & RA & $\begin{array}{l}\text { Dispatcher: Min [Total travel cost \& population } \\
\text { exposure \& environmental risk] }\end{array}$ & - & DC & $\mathrm{Y} / \mathrm{N}$ & - & $S$ & $\mathrm{~N}$ & $\mathrm{M}$ & LB & $\mathrm{H}$ \\
\hline Karbassi Yazdi et al. (2019) & MA & Dispatcher: Min [Total travel cost] & - & MO & $\mathrm{N} / \mathrm{N}$ & - & $\mathrm{S}$ & $\mathrm{N}$ & $S$ & LB & $\begin{array}{c}\mathrm{MH} \\
(\mathrm{PSO})\end{array}$ \\
\hline Liu et al. (2019) & RO & Dispatcher: Min [Total travel time \& expected risk] & - & $\mathrm{SO}$ & $\mathrm{Y} / \mathrm{N}$ & - & $\mathrm{S}$ & $\mathrm{N}$ & $\mathrm{M}$ & LB & $\mathrm{E}$ \\
\hline Timajchi et al. (2019) & RO & $\begin{array}{l}\text { Dispatcher: Min [Total travel cost \& maximum expected } \\
\text { risk] }\end{array}$ & - & DC & $\mathrm{N} / \mathrm{N}$ & $\mathrm{K}$ & $S$ & $\mathrm{Y}$ & $\mathrm{M} \mid$ & LB & $\mathrm{MH}(\mathrm{G})$ \\
\hline Jiang et al. (2020) & RO & $\begin{array}{l}\text { Dispatcher: Min [Total travel cost \& expected risk } \\
\text { \&average vehicle redundancy] }\end{array}$ & - & DC & $\mathrm{Y} / \mathrm{N}$ & $\mathrm{K}$ & $S$ & $\mathrm{Y}$ & $\mathrm{M}$ & LB & $\begin{array}{c}\mathrm{MH} \\
(\mathrm{VNS})\end{array}$ \\
\hline Men et al. (2020) & RO & $\begin{array}{l}\text { Dispatcher: Min [An uncertain risk \& number of } \\
\text { vehicles] }\end{array}$ & - & DC & $\mathrm{N} / \mathrm{N}$ & $\mathrm{K}$ & $S$ & $\mathrm{Y}$ & $\mathrm{M}$ & LB & $\mathrm{MH}(\mathrm{G})$ \\
\hline Ouertani et al. (2020) & RO & Dispatcher: Min [Total travel cost \& expected risk] & - & DC & $\mathrm{N} / \mathrm{N}$ & $\mathrm{K}$ & $\mathrm{S}$ & $\mathrm{N}$ & $\mathrm{S}$ & LB & $\begin{array}{c}\mathrm{MH} \\
(\mathrm{G} \& \mathrm{VNS})\end{array}$ \\
\hline Current study & RO & $\begin{array}{l}\text { Dispatcher: Min [Total travel time \& expected risk] } \\
\text { Demon: Max [Total expected risk] }\end{array}$ & St & MO & $\mathrm{Y} / \mathrm{Y}$ & UN & $\mathbf{M}$ & $\mathrm{Y}$ & $\mathrm{M}$ & LB & $\begin{array}{c}\text { MH } \\
\text { (ALNS } \\
\& \text { SA) } \\
\end{array}$ \\
\hline
\end{tabular}

Alphabetical sorting of acronyms: ACO: Ant Colony Optimization; DC: Depot \& Customers; E: Exact; G: Genetic; H: Heuristic; K: Known;

LB: Link-Based; M: Multi; MA: Maritime; MH: Metaheuristic; MO: Multiple ODs; N: No; Na: Nash; PSO: Particle Swarm Optimization; RB: Route-Based; RA: Rail; RO: Road; S: Single; SO: Single OD; St: Stackelberg; TS: Tabu Search; UN: Unknown; VNS: Variable Neighborhood Search; Y: Yes. 
Main realistic assumptions: The time and location of hazmat incidents in a road network are uncertain. To apply a reliable density function to represent the incident probability of a road, a sufficient number of historical records is required. Up to 2012, the majority of researchers assumed known incident probabilities for roads of their networks. However, in reality, rarely we can find a transportation network with sufficient historical data for "all" road links to quantify the probability of incidents (note that we may have sufficient historical data only for some- not all- links). Therefore, for the first time, Hosseinloo et al. (2012) assumed that incident probabilities are unknown. Later, Szeto (2013) and Szeto et al. (2017) relied on the same assumption in their modeling. To deal with an unknown incident probability, non-cooperative game theory was used in the literature (Hosseinloo et al., 2012; Szeto, 2013; Szeto et al., 2017). The game is often between carriers and an imaginary attacker (i.e. a virtual demon). In this research, we follow this practical trend of the literature and consider unknown incident probabilities for the roads of the network.

Objective functions: There are two most popular groups for objective functions considered in hazmat routingscheduling: i) economic objective functions, such as minimizing travel costs or travel distance/time; and ii) riskbased objective functions, such as minimizing the incident probability or equitable risk spread over the investigated area. As Table 1 shows, the previous papers are either single-objective (e.g. Szeto et al., 2017; Fang et al., 2017; Hu et al., 2017a) or multi-objective (e.g. Abuobidalla et al., 2019; Liu et al., 2019; Jiang et al., 2020).

Among the references with a single-objective function, only Fang et al. (2017) focused on a single economic objective function while others considered a single risk-based objective function. The demon approach was investigated only by Hosseinloo et al. (2012) in a multi-objective hazmat routing-scheduling with both economic and risk-related objective functions. However, the research was established a Nash game between the demon and carriers as the major player of the hazmat routing-scheduling problem. In this research, we investigate the demon approach in a multi-objective hazmat routing-scheduling problem with both economic and risk-related objective functions but the carriers (as the leader) play a Stackelberg game with the demon(s) (as the follower).

Complexity: Hazmat routing-scheduling problems are computationally complex. The last column of Table 1 illustrates that the majority of scholars exploited heuristic and meta-heuristic techniques to tackle problems that are much simpler than our research problem. Note that the importance of computational time in such problems is not only because of their complexity. The hazmat routing-scheduling problem is a tactical/ operational (not a strategic) decision. Therefore, the related models or solution techniques should be solvable in a short computational time, even for large-scale problems. Therefore, heuristic and meta-heuristic techniques are usually designed to solve these models rather than exact solution approaches. In the literature, five papers used exact solutions: Toumazis and Kwon (2013), Faghih-Roohi et al. (2016), Szeto et al. (2017), Hsu et al. (2018), and Liu et al. (2019). Only simplified small or medium-size problems can be globally solved with exact approaches.

In this research, we have formulated an exact model which is link-based. The formulation is useful to solve small and some medium-size problems (Szeto et al., 2017). The papers other than Szeto et al. (2017) exploited a routebased modeling approach that needs exhaustive route enumeration, which is usually done through a column generation technique. Szeto et al. (2017) showed that the complexity of the link-based model is less than its routebased version. Therefore, we take the link-based approach to enable us to solve a broader range of problems optimally. For large-size problems, we have developed a modified metaheuristic method based on ALNS and SA.

\subsection{Contribution}

This paper contributes to the hazmat routing-scheduling literature in the following ways:

FIFO queueing: The time-dependent hazmat routing and scheduling will result in different routes and schedules 
for dispatchers. Moreover, there can be travel restrictions (curfews) for some links and nodes. Therefore, there is no guarantee that the vehicles departing earlier from their origins arrive earlier at their destinations; this is not fair to the dispatchers. To solve this issue, we enforce the FIFO discipline on the model. The importance of FIFO discipline as a critical behavioral aspect has been discussed in the literature. According to Larson (1987), “... a lengthy, but orderly, wait in a queue does not bother people as much as the occasional violation of the FIFO discipline." It is referred to as social justice in Gopalan et al. (1990); it is highlighted that instead of merely focusing on optimization aspects of a queuing problem, a careful study of attributes related to "social justice" must be captured. Choosing the same route by different drivers is similar to a single-line system. In a single-line system, FIFO is guaranteed. Schmitt et al. (1992) stated that in multiline systems, FIFO is not guaranteed. Such queues elicit negative responses towards intrusion, which is referred to as social injustice. Zhou and Soman (2008) highlighted the adverse reactions of stakeholders (e.g., customers) in terms of satisfaction. Note that in a hazmat routing-scheduling problem with such a setting, trucks need to wait in some locations (e.g., similar to trains that stop in some points to avoid collision). Practically, incidents can still happen when trucks stop. Therefore, some safe areas in non-residential areas or safe places (e.g., under-ground places or protected areas) are identified to increase the safety level. Observing the FIFO discipline (regarding travel time) among dispatchers has not been studied in the literature of hazmat routingscheduling; we address this gap.

A special-purpose game: Using a game-theoretical concept helps us consider the uncertainty aspect related to unknown incident probabilities. We consider a virtual game played between the dispatcher company- who attempts to move shipments safely- and an imaginary demon (or more) (i.e. an evil entity that intentionally causes damage) who intends to attack the shipments to cause the maximum possible injuries and fatalities. Looking at the demon as an intelligent attacker has already been employed, not only in hazmat routing-scheduling problems (refer to Bell, 2006; Szeto, 2013; Szeto et al., 2017) but also in other infrastructure safety and security research studies (e.g., Yates, 2008; Yates et al., 2011; Yates and Casas, 2012; Yates et al., 2012). The impact of hazmat incidents is similar to mass destruction weapons in the hands of an adversary (Huang et al., 2004). That is why we can look at the problem as a game played between a planner and an imaginary attacker. Bell (2006), Szeto (2013) and Szeto et al. (2017) have already applied the game-theoretical concept, in which a dispatching company and demons play simultaneously, but their research is based on a Nash game for the worst-case scenario. The Nash game has been criticized in practice because (1) first, the dispatcher decides the optimum route and schedule and (2) then, the demon decides to cause the incident(s). In other words, the dispatcher has the first-mover advantage; this will end up in a model with a Stackelberg game in which the dispatching company is the leader and the demon(s) is (are) the follower(s). Moreover, the players play the game over a risk objective function. At the same time, we also observe a travel time objective for the dispatcher in a bi-objective context. This helps us study trade-offs between risk and travel time. Considering a leader-follower game in which the leader observes two objective functions while the follower only optimizes one objective function, is one of the contributions of this research.

Methodology: In this research, not only have we considered many complicating aspects in the research (e.g., multi-hazmat class, multi-vehicle type, multi-period, etc.), to the best of our knowledge, the dependence of links' travel times on all of these factors simultaneously has been considered in the research for the first time. The abovementioned contributions, in addition to these realistic assumptions, make the problem computationally more complex than the other research studies in the area. Therefore, we need to design an efficient solution approach to solve large-scale problems. In this paper, a modified ALNS algorithm is designed, which is embedded in a local search algorithm based on SA. The modifications (as a contribution of the research) are related to the processes of 
(i) selecting removal and insertion moves and (ii) accepting a new solution for each iteration of the algorithm to speed up the process and avoid many unfeasible solutions. The technical details of the methodological contributions will be explained in section 5 .

To the best of our knowledge, the impact of the number of each vehicle type used in dispatching hazmat cargoes on the tradeoff between economic and safety objectives has not been studied in the literature.

\section{PROBLEM DEFINITION}

We consider a transportation company that plans for the shipment of different classes of hazmat cargos between multiple OD pairs in a transportation network. The demand for each hazmat class between each OD pair is known and the company is supposed to meet these demands during a multi-period time horizon with its own limited number of heterogeneous vehicles (e.g., tanker trucks). Therefore, the transportation vehicle capacity of the company within the time horizon is limited. Obviously, this constraint can be relaxed by considering a large number of vehicles to convert the problem into an un-capacitated version. For the ease of problem definition, we define and use the term "dispatcher" in this research. A dispatcher refers to a fleet with a limited number of heterogeneous vehicles, which are in charge of the movement of a specific hazmat class between a specific OD pair to fulfill the related transportation demand. Note that, practically, the same vehicles cannot be used for the movement of different hazmat classes. In practice, the decisions made by the transportation company are at the vehicle level rather than the dispatcher level. This means the transportation company makes routing and scheduling decisions (i.e. departure and stop times) for each vehicle so that the demand for each dispatcher is satisfied. Figure 1 illustrates the definition of the problem:

\begin{tabular}{l} 
Problem Definition \\
A Stackelberg Game \\
Model's Objectives \\
Model's Formulation \\
Incident probability \\
Queuing Discipline \\
Hazmat Classes \\
Vehicle Types \\
Fleet Size \\
Solution Algorithm \\
\multirow{2}{\leftarrow}{$=$ Contribution }
\end{tabular}
A routing-scheduling problem with possibility of waiting on routes Leader: A transportation company, Follower: Multi virtual demons Leader: Minimizing (1) cost, (2) risk Follower: Maximizing loss

\begin{tabular}{|c|c|c|c|}
\hline Route-based & $x$ & Link-based & $\checkmark$ \\
\hline Known & $x$ & Unknown & $\checkmark$ \\
\hline No & $x$ & Yes: FIFO & $\checkmark \quad$ 市 \\
\hline Single & $x$ & Multi & $\checkmark$ \\
\hline Single & $x$ & Multi & $\checkmark$ \\
\hline Unlimited & $x$ & Limited & $\checkmark$ \\
\hline
\end{tabular}

Figure 1. The specific features of the proposed problem.

To avoid incidents and minimize risk, the dispatchers may use different routes and periods to accomplish shipments; otherwise, the flow on a link will be high and a possible incident will cause a severe incident. This distribution of flows may cause unfairness among vehicles because there is no guarantee that a vehicle departing from an origin before the others will arrive at the destination earlier. In other words, in this case, the FIFO discipline is not guaranteed. To resolve this issue, we force the model to consider the FIFO discipline among all vehicles by keeping some vehicles waiting on the waiting links (as queueing links). Note that considering FIFO is a rather important issue, as the time-dependent routing-scheduling will result in different waiting times and, consequently, in different risks. The impact of applying FIFO to travel time and risk objective functions is investigated in this research. Note that in the optimal solution, the model may find different routes and schedules for different vehicles, even if they belong to the same dispatcher. 


\subsection{Space-Time Expanded Network}

In this research, we use the concept of Space-Time Extension Network (STEN) (Drissi-Kaïtouni et al., 1992) to schematically model the transportation network while time-periods are embedded in it. Using STEN markedly simplifies the concurrent routing and scheduling of hazmat cargos in both route- and link-based approaches. The transportation network is represented as $G=[N, A]$ in which $N$ is the set of nodes and $A$ is the set of directed links. Each link corresponds to a road segment in the network. It should be noted that although modeling a network with short links results in increasing the size of the problem due to the long real road links needed to be separated to some short links by some virtual nodes, it increases the accuracy of the results. The nodes of the network consist of three subsets, namely, origin nodes $\left(N_{o}\right)$, destination nodes $\left(N_{d}\right)$ and intermediate nodes $\left(N_{i}\right)\left(N=N_{o} \cup N_{d} \cup N_{i}\right)$.
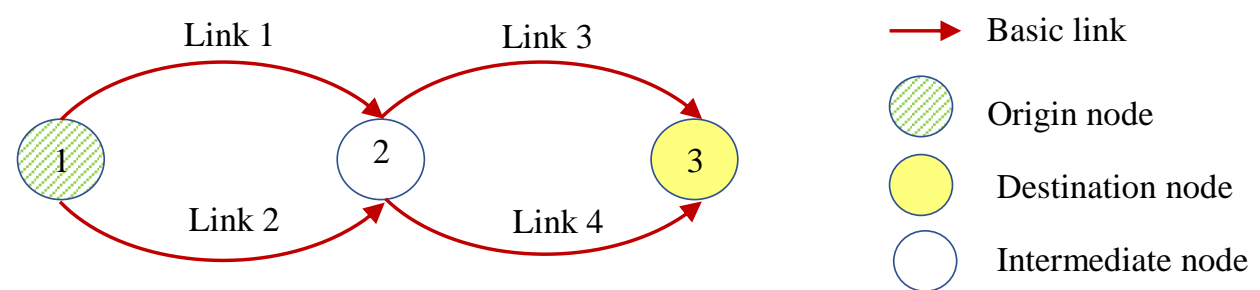

Figure 2. The original directed network.

Figure 2 shows a simple road network where the links replicate the road's real-world transportation network. Figure 3 shows the equivalent STEN of the original network in Figure 2, with one OD in a planning horizon of three periods. The dispatcher must start the journey from an origin node (here, node 1), move through the intermediate node(s) (here, node 2) and stop at the planned destination node (here, node 3) to deliver the identified hazmat and eventually fulfill the promised demand. To convert the network of Figure 2 into a STEN, it should be repeated $T$ times, where $T$ shows the number of time-periods within the planning horizon. Each repetition of the original network is labeled as $G^{t}=\left[N^{t}, A^{t}\right]$, where $N^{t}$ and $A^{t}$ represent the sets of nodes and links at period $t$, respectively. The connections between the repeated networks are provided with a set of dummy origin nodes $\left(R^{\prime}\right)$, a set of dummy destination nodes $\left(S^{\prime}\right)$, a set of dummy links $\left(A_{d}\right)$ and a set of waiting links $\left(A_{w}\right)$. There is a dummy origin/ destination node that corresponds to each origin/ destination node in the original network connecting all origins/ destinations in the repeated $G^{t}$ networks. Overall, there are $\left|N_{o}\right|+\left|N_{d}\right|$ dummy nodes in the STEN. Therefore, the number of dummy links in the STEN $\left(\left|A_{d}\right|\right)$ is equal to $\left(\left|N_{o}\right|+\left|N_{d}\right|\right) \times T$. The dummy links determine the departure and arrival times of the origin and destination nodes. In addition to the dummy links, there are some waiting links in the STEN that provide an extra choice for the routing and scheduling of hazmat cargos so that a dispatcher can stop at a particular node in any period to continue its journey in future periods. Therefore, a waiting link connects $G^{t}$ to $G^{t+1}(t=1,2, \ldots, T-1)$. The STEN is represented by $G^{\prime}=\left[N^{\prime}, A^{\prime}\right]$ so that $N^{\prime}=\left\{\left(\cup_{t=1}^{T} N^{t}\right) \cup S^{\prime} \cup R^{\prime}\right\}$ and $A^{\prime}=$ $\left\{\left(\cup_{t=1}^{T} A^{t}\right) \cup A_{d} \cup A_{w}\right\}$. 


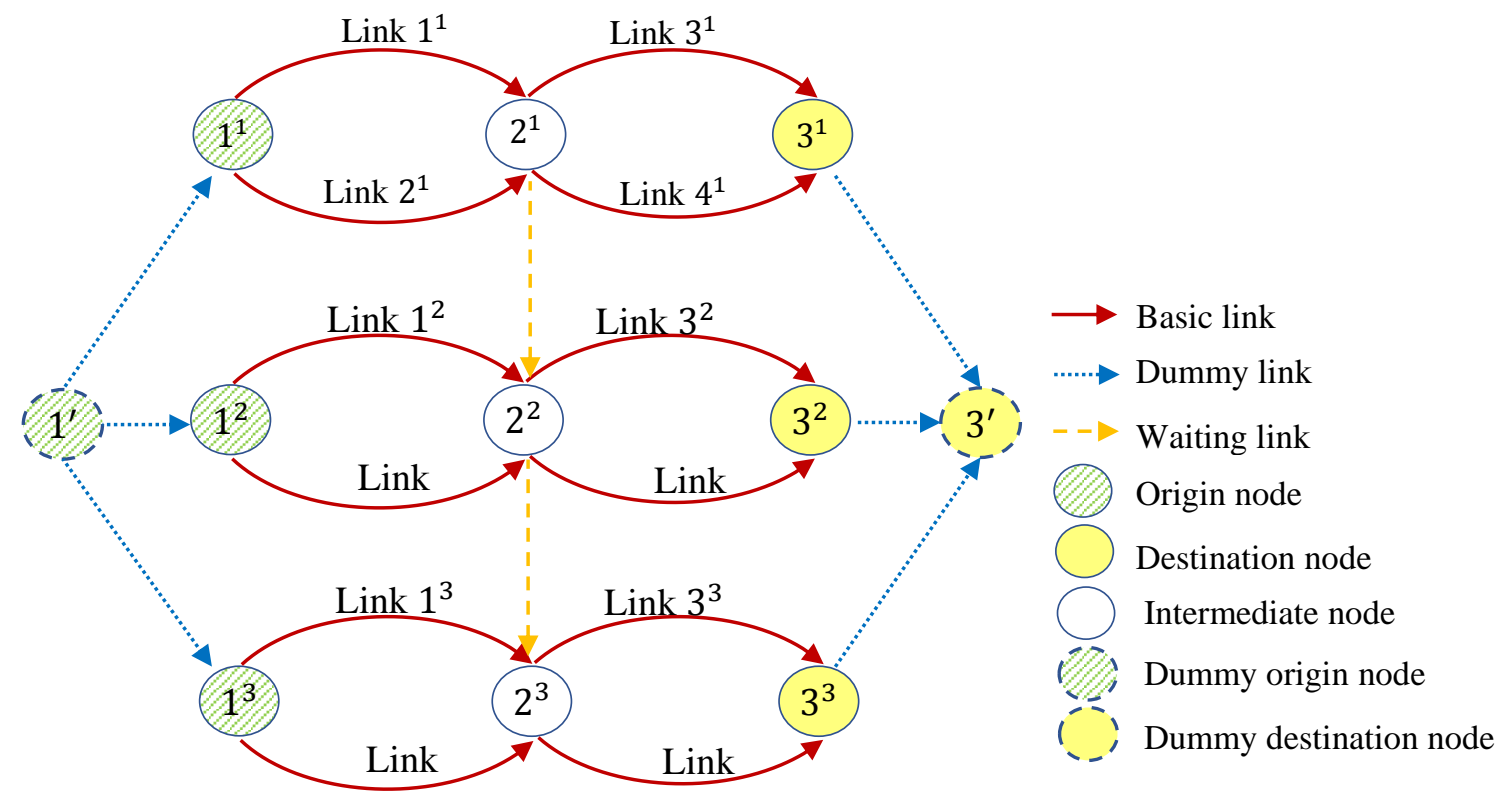

Figure 3. The equivalent STEN of the network shown in Figure 2.

\subsection{The Transportation Company (Leader) Problem}

Due to the lack of sufficient historical incident data on all (or some) links of the transportation network, we consider the incident probability of the links in the network to be unknown. Please note that we consider an urban road network with thousands of links, in many of which historically no or few incidents have been observed or recorded. Such a level of historical data does not mean these links are safer than the others. In other words, the lack of sufficient historical data does not allow us to calculate the probability of incidents on all routes precisely. In this study, both economic and risk-related objective functions are considered by the transportation company. The company determines the appropriate routes and schedules for the vehicles of the dispatchers in a way that optimizes two objectives: (1) minimizing the total travel time, which is a proxy of the total transportation cost, and (2) minimizing the expected loss, which is a proxy of risk to residential areas in the vicinity of the possible location of the incident(s). For any given route and schedule, the risk is calculated in terms of the hazmat type, the amount of hazmat in the vehicle and the expected number of people who are subject to the danger in the vicinity of the potential incident location.

We assume that all the hazmat cargos are available at time zero at their corresponding origin nodes of the network for shipping to the demand destinations. We consider a finite time horizon (e.g., length of a working day for as long as the vehicles are allowed to travel through residential areas (e.g., during daylight)). In the STEN, the time horizon is divided into some shorter time-periods. Since traffic flow on links of a network is dynamic during the day, we assume the travel time of any given link changes from one period to another. In this research, we split the time horizon into a number of periods to (1) consider the dynamic nature of traffic flow during the time horizon, which will affect the travel time in different time-periods and (2) investigate their impact on the optimal objective values. Increasing the number of time-periods increases our precision in planning and reduces the vehicles' waiting time; however, it needs more detailed traffic data and ends up with a more complicated STEN.

According to the problem characteristics and considerations, the transportation company is responsible for making the following decisions:

- departure times for vehicles;

- optimal OD routes for the vehicles;

- waiting stations of each vehicle on its route toward the destination; and 
- type and number of hazmat vehicles (small, medium and large) on each of the selected routes.

\subsection{The Demons (Follower) Problem}

The serious consequences of hazmat incidents force decision makers to be fully or partially risk averse. We exemplify hazmat incidents with attacks optimally planned by imaginary demons to maximize the risk of the dispatchers. In this case, the problem is modeled as a non-cooperative Stackelberg game between two groups of players: a) the dispatchers (as the leader), where the transportation company focally optimizes routing and scheduling decisions; and b) the imaginary demons (as the follower), who optimally cause incidents in the network's links. Therefore, this is a non-zero bi-level game played between the two players. The issue is that such a model considers the worst-case scenario in which only risk objective function is valued. To solve this issue, we consider (i) two objective functions for the dispatcher (i.e. risk and travel time) and (ii) one objective function for the demon(s) (i.e. risk). The two players play the game only over the risk objective and the other objective (i.e. travel time) only belongs to the dispatcher. Adjusting the weight of each objective function according to the company's risk attitude suggests the optimal solution.

Assume that hazmat incidents are planned by $M$ demons because the probability of more than one incident occurring is high in multi-period routing and scheduling problems. Each demon attacks no more than one link to cause an explosion when the hazmat vehicle is passing through it. The potential population that could be affected by the explosion of each link is known for the demons. Each demon seeks an optimal link to attack. The choices of the demons are made independently. Each demon has $\left|A^{\prime}\right|$ attack choices. Overall, $\left|A^{\prime}\right|^{M}$ attack scenarios are potentially available for all demons. The demons intend to maximize the overall risk, which is equal to the total expected number of the affected population.

There are two flexibilities embedded in the problem to model the preferences and behavior of the dispatchers: (i) The dispatchers can investigate trade-offs between the two objective functions by adjusting their weights depending on the risk attitude of the decision makers (i.e. risk averse, risk seeking and risk neutral); an overly riskseeking company will overweigh the travel time objective and vice versa; (ii) The number of demons can be changed to show how much the dispatchers' routing-scheduling plan is prepared for incidents. In the research, we consider the existence of at least one demon. For a risk-averse transportation company, we can increase the number of demons incrementally (i.e. the preparedness level) to analyze the consequences and the impact on the objective functions. Additionally, we will conduct some other sensitivity analyses over various aspects of the problem to extract interesting insights for practitioners.

\section{MODEL FORMULATION}

In this section, we formulate the Stackelberg game played between the demons and dispatchers. As noted earlier, the focal company (or, say, dispatchers collaboratively) is the leader trying to find the route and schedule movement of consignments to minimize the risk and travel cost. This model includes different types of vehicles, capacity limitation of the fleet size of each dispatcher and FIFO discipline among dispatchers. The demons are the follower intending to select and attack links to maximize their overall payoffs, equal to the dispatchers' loss. This game leads to a bi-level programming model. The outer level of the model belongs to the dispatchers and the inner level belongs to the demons. In the following, a closed-form expression of the proposed model is presented. Subsequently, the definition and the methodology behind each equation in the model are explained in detail. The notation used in the model and other parts of the paper is introduced in Appendix A.

Traditionally, link- and route-based approaches are used to develop models for routing hazmat cargos (Szeto et 
al., 2017). The route-based models need an explicit path enumeration before solving the model. In the path enumeration step, a set of paths or K-shortest paths between each OD pair is needed (Van der Zijpp and Catalano, 2005). This step significantly increases the complexity of route-based models (Cascetta et al., 1997) and increases computational efforts for large-scale transportation networks (Bell, 2006; Chen and Hsueh, 1998; Ran and Boyce, 1996; Ran et al., 1996; Szeto et al., 2017). Ran et al. (1996) showed that even in simple grid networks, the number of routes increases exponentially by increasing the number of nodes. Therefore, in this research, we use a link-based approach in our models for two reasons: (1) its modeling complexity is lower than the route-based version and (2) it is easier to code and run on commercial optimization software products such as GAMS and LINGO because the preliminary route enumeration is not required. The models can be used to find globally optimal solutions in smalland medium-scale transportation networks. Note that while neither route-based nor link-based formulations can solve large-scale problems (and eventually we will be in need of a heuristic or meta-heuristic algorithm to solve large-scale problems), the link-based formulation helps us solve larger test problems than its route-based counterpart in the validation stage. The same logic led us to embed a link-based concept in designing our metaheuristic algorithm to solve real-life, large-scale problems.

\section{The leader's subproblem:}

$$
\begin{aligned}
& \operatorname{Min} \sum_{a^{t}=(x, y)} \eta_{a^{t}} \\
& \operatorname{Min} \sum_{r s_{q}} \sum_{f} \sum_{x, x=D^{\prime}\left(r s_{q}\right)} \sigma_{x}^{r s_{q f}}
\end{aligned}
$$

s.t.

$$
\begin{array}{ll}
C_{a^{t}}(V)=0 & \forall a^{t} \in A_{d} \\
C_{a^{t}}(V)=C_{x} & \forall a^{t}=(x, y) \in A_{w} \\
C_{a^{t}}(V)=\sum_{r s_{q}} \sum_{f} \beta_{a^{t}}^{Q^{\prime}\left(r s_{q}\right) f}\left(v_{a^{t}=(x, y)}^{r s_{q f}}\right)^{\alpha_{a^{t}}^{Q^{\prime}\left(r s_{q}\right) f}} & \forall a^{t} \in \cup_{t=1}^{T} A^{t} \\
\eta_{a^{t}=\sum_{s} u_{s} C_{l^{t} m}(V)=x_{a^{t}} C_{a^{t}}(V)} & \left(\forall a^{t}=(x, y), r s_{q}=1,2, \ldots, H\right) \\
x_{a^{t}}=1-\prod_{i=1}^{M}\left(1-p_{l_{m}^{t}}\right) & \left(\forall a^{t}=(x, y) \in A\right) \\
\sum_{f} \sum_{a^{t}, O\left(a^{t}\right)=x} v_{a^{t}}^{r s_{q f}} w_{f}-\sum_{f} \sum_{a^{t}, D\left(a^{t}\right)=x} v_{(y, x)}^{r s_{q f}} w_{f}=Q_{x}^{r s_{q}} & \left(\forall x, r s_{q}\right)
\end{array}
$$

$Q_{x}^{r s_{q}}=\left\{\begin{array}{cl}d^{r s_{q}} & x=O^{\prime}\left(r s_{q}\right) \\ -d^{r s_{q}} & x=D^{\prime}\left(r s_{q}\right) \\ 0 & \text { otherwise }\end{array}\right.$

$\sum_{a^{t}, O\left(a^{t}\right)=x} v_{a^{t}}^{r s_{q f}}-\sum_{a^{t}, D\left(a^{t}\right)=x} v_{a^{t}}^{r s_{q f}}=0$

$\left(\forall x \neq\left\{O^{\prime}\left(r s_{q}\right), D^{\prime}\left(r s_{q}\right)\right\}, f, r s_{q}\right)$

$v_{a^{t}}^{r s_{q f}} \leq Z_{a^{t}}^{r s_{q f}} \times U$

$v_{a^{t}}^{r s_{q f}} \geq Z_{a^{t}}^{r s_{q f}} \times L$

$\sigma_{x}^{r s s_{q f}}={\sigma^{\prime}}_{x}^{r s_{q f}}={\sigma^{\prime \prime}}_{x}^{r s_{q f}}=0$

$\sigma_{x}^{r s_{q f}}=\sigma_{x}^{\prime r s_{q f}}+\sigma^{\prime \prime}{ }_{x}^{r s_{q f}}$

${\sigma^{\prime}}_{x}^{r s_{q f}}=\sum_{a^{t} \notin A_{w}, D\left(a^{t}\right)=x, O\left(a^{t}\right)=i}\left({\sigma^{\prime}}_{i}^{r s_{q f}}+t_{a^{t}}^{r s_{q f}} v_{a^{t}}^{r s_{q f}}\right) Z_{a^{t}}^{r s_{q f}}$

$\left(\forall f, r s_{q}, x \& x \in\left\{O^{\prime}\left(r s_{q}\right), N_{o}\right\}\right)$

$\sigma^{\prime \prime}{ }_{x}^{r s_{q f}}=\sum_{a^{t}, D\left(a^{t}\right)=x, O\left(a^{t}\right)=i}{\sigma^{\prime \prime}}_{i}^{r s_{q f}} Z_{a^{t}}^{r s_{q f}}+\sum_{a^{t} \in A_{w}, D\left(a^{t}\right)=x, O\left(a^{t}\right)=i}\left(D+\lambda^{q f}\right) v_{a^{t}}^{r s_{q f}}$

$\left(\forall x, f, r s_{q} \& x \notin\left\{O^{\prime}\left(r s_{q}\right), N_{o}\right\}\right)$

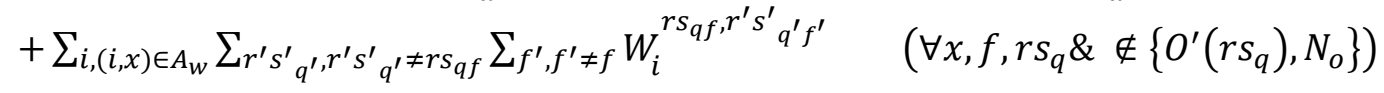

$\frac{\sigma_{i}^{r^{\prime} s^{\prime}} q^{\prime} f^{\prime}}{v_{a^{t}}^{r^{\prime} s^{\prime} q^{\prime} f^{\prime}+L}}-\frac{\sigma_{i}^{r s} q f}{v_{a^{t}}^{r s f}+L} \geq-U \times \mathrm{Y}_{i}^{r^{\prime} s^{\prime}}{ }_{q^{\prime} f^{\prime}, r s_{q f}}$

$$
\left(\forall a^{t}, f, f^{\prime}, r s_{q}, r^{\prime} s^{\prime}{ }_{q^{\prime}} \text { and } r^{\prime} s^{\prime}{ }_{q^{\prime}} \neq r s_{q}, f \neq f^{\prime}, a^{t} \in A_{w}, O\left(a^{t}\right)=i\right)
$$

$\frac{\sigma_{i}^{r^{\prime} s^{\prime}{ }_{q^{\prime} f^{\prime}}}}{v_{a^{t} s^{t} q^{\prime} f^{\prime}+L}}-\frac{\sigma_{i}^{r s} q f}{v_{a^{t}}^{r s}+L} \leq U \times\left(1-Y_{i}^{r^{\prime} s^{\prime}{ }_{q^{\prime} f^{\prime}}, r s_{q f}}\right)$

$$
\left(\forall a^{t}, f, f^{\prime}, r s_{q}, r^{\prime} s^{\prime}{ }_{q^{\prime}} \text { and } r^{\prime} s^{\prime}{ }_{q^{\prime}} \neq r s_{q}, f \neq f^{\prime}, a^{t} \in A_{w}, O\left(a^{t}\right)=i\right)
$$




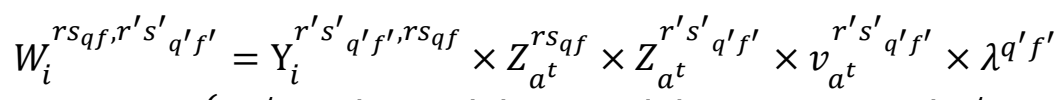

$$
\begin{aligned}
& \left(\forall a^{t}, f, f^{\prime}, r s_{q}, r^{\prime} s^{\prime}{ }_{q^{\prime}} \text { and } r^{\prime} s^{\prime}{ }_{q^{\prime}} \neq r s_{q}, f \neq f^{\prime}, a^{t} \in A_{w}, O\left(a^{t}\right)=i\right) \\
& v_{a^{t}}^{r s_{q f}} \leq F^{r s_{q f}}-\sum_{a^{\prime}, a^{\prime}{ }^{t} \in A_{w}, b\left(a^{\prime}\right)=b\left(a^{t}\right)-1} v_{a^{\prime t}}^{r s_{q f}} \quad\left(\forall f, r s_{q}, a^{t}, O\left(a^{t}\right) \in S^{\prime}\right)
\end{aligned}
$$

The follower's subproblem:

$$
\begin{gathered}
\operatorname{Max} P=\sum_{s} \sum_{a^{t}} u_{s} C_{a^{t}}(V) \\
(3),(4),(5), \text { and } \\
u_{s}=\prod_{m=1}^{M} p_{l^{t}} \\
C_{a^{t}{ }_{s}}(V)=\left\{\begin{array}{c}
0 \\
C_{a^{t}}(V) \\
p_{l^{t}{ }_{m}} \geq 0 \\
\sum_{l^{t}{ }_{m}} p_{l^{t}{ }_{m}}=1
\end{array}\right.
\end{gathered}
$$$$
\text { if } a^{t} \notin s
$$$$
\text { otherwise, }
$$

First, the equations of the follower's subproblem are described to shed light on the definition of some equations in the leader's subproblem. In addition, note that the formulation of the follower's subproblem follows Szeto et al. (2017), but the rest are unique to this research. The follower's subproblem consists of $M$ demons. Each demon has $\left|A^{\prime}\right|$ choices to select and attack links. In the problem with $M$ demons, in total $\left|A^{\prime}\right|^{M}$ scenarios can be defined for selecting and attacking links by the demons. Set $S=\{s\}$ includes these scenarios. Scenario $s=\left(l^{t}{ }_{1}, \ldots, l^{t}{ }_{m}, \ldots, l^{t}{ }_{M}\right)$ includes the links that are attacked by $M$ demons. $p_{l^{t}}$ is defined as the probability that demon $m$ attacks link $l^{t}$. The demons select their attack links independently. Therefore, the probability of scenario $s=\left(l^{t}{ }_{1}, \ldots, l^{t}{ }_{m}, \ldots, l^{t}{ }_{M}\right)$ is as equation (22). According to equation (22), if two demons attack a link with a probability of $100 \%$, the loss of the link will not be doubled. Therefore, the demons select separate links to attack.

According to equation (23), if link $a^{t} \in A^{\prime}$ is not chosen by any of $M$ demons in scenario $s$, the loss of link $a^{t} \in$ $A^{\prime}$ in scenario $s, C_{a^{t} s}$, is equal to zero; otherwise, it is equal to $C_{a^{t}}(V)$. As the purpose of this study is planning for the worst-case scenario, for an original link $a^{t}$, a maximum loss, $C_{a^{t}}(V)$, is defined. The maximum loss along a link represents the number of affected people if an incident happens at a point in the link. Most of the previous studies consider a uniform population around the links to calculate consequences. We disregard this uniformity and calculate the consequences based on the segment of the link that results in a maximum loss.

Therefore, the total expected payoff caused by the demons is formulated as objective (21). According to (24), the probability of selecting a network's link by a demon is a non-negative value. If demon $m$ causes an incident on link $l_{m}^{t}$, then $p_{l^{t}} \geq 0$; otherwise, it must be zero. Condition (25) ensures that the total incident probability assigned to the network links by each demon must be equal to 1 . Conditions (3), (4) and (5) show the loss of link $a^{t}$. The amount of loss of a link depends on its type (original links, dummy or waiting links of STEN). In equation (3), the loss of dummy links is equal to zero. In (4), the loss of a waiting link depends on the maximum loss of its starting node $(x)$. We assume that the loss of a waiting link depends on the loss of its starting node. Other assumptions can be made to determine the loss of waiting links. Below, we show how condition (4) can be modified for other assumptions about the loss of waiting links. When the FIFO discipline is applied in waiting links, the vehicles waiting on a link will not leave the link instantly at the beginning of the next period. Therefore, the waiting time can be shared between the starting and ending nodes of the waiting link. Accordingly, conditions (26) or (27) can be used instead of (4) in case the loss of a waiting link is the average or maximum loss of its starting and ending nodes:

$$
\begin{array}{ll}
C_{a^{t}}(V)=\frac{C_{x}+C_{y}}{2} & \forall a^{t}=(x, y) \in A_{w} \\
C_{a^{t}}(V)=\max \left\{C_{x}, C_{y}\right\} & \forall a^{t}=(x, y) \in A_{w}
\end{array}
$$

Note that (5) is suitable if queueing discipline is not applied to waiting links. In (26), queueing discipline is applied to waiting links but we do not consider the worst-case scenario. (27) is suitable when queueing discipline is 
applied to the waiting links and we are seeking the worst-case scenario. Without loss of generality, we use condition (27). Condition (5) shows the loss of the original links in STEN. $\alpha_{a^{t}}^{q f}\left(\right.$ or $\alpha_{a^{t}}^{Q^{\prime}\left(r s_{q}\right) f}$ ) and $\beta_{a^{t}}^{q f}\left(\right.$ or $\beta_{a^{t}}^{Q^{\prime}\left(r s_{q}\right) f}$ ) are parameters determining the worst-case loss for links. The worst-case loss of link $a^{t}$ depends on the characteristics of the link $\left(\alpha_{a^{t}}^{Q^{\prime}\left(r s_{q}\right) f}\right.$ and $\left.\beta_{a^{t}}^{Q^{\prime}\left(r s_{q}\right) f}\right)$, the class of hazmat $(q)$, the flow of the consignment $\left(v_{a^{t}}^{r s_{q f}}\right)$, the type of vehicle used for transportation $(f)$, population density and the incident impact radius.

Objective functions (1) and (2) minimize the risk and travel time of dispatchers, respectively. As explained above, constraints (3), (4) and (5) compute the total loss for the network's links. Constraint (6) calculates the risk of each dispatcher. Based on (21) and (22), we can demonstrate that this equation holds in this constraint: $\sum_{s} u_{s} C_{a^{t}}(V)=x_{a^{t}} C_{a^{t}}{ }^{r s_{q}}(V) . x_{a^{t}}$ shows the probability that link $a^{t}$ is selected by at least one demon and is calculated by constraint (7). Flow conservation balance at the nodes of the network is guaranteed by constraints (8) and (9). In constraint (8), $w_{f}$ is the capacity of vehicle type $f$. The constraints ensure that the total in-flow of each dispatcher is equal to its total out-flow at each node. Constraint (10) ensures that the total number of vehicles of each type entering into each node (except the dummy origin and destination nodes) should be equal to the total number of vehicles of the same type leaving that node. Constraints (11) and (12) ensure that the binary variable $Z_{a^{t}}^{r s_{q f}}$ must be equal to 1 when variable $v_{a^{t}}^{r s_{q f}}$ is positive; otherwise, it would be zero. Constraints (13) through (16) compute the total accumulated travel time for dispatcher $r s_{q}$ using vehicle type $f$ from its origin node $(r)$ to node $x$. In Appendix $\mathrm{B}$, the necessary explanations about how the constraints work are presented.

FIFO Constraints: Constraint (16) is composed of three terms. The first term represents the time added by the prior waiting links of the network. The second term shows the time added by the waiting links without considering the delay caused by other vehicles due to the FIFO discipline. Finally, the third term adds the delay caused by the vehicles of other dispatchers due to the FIFO discipline. The total delay imposed by a vehicle type $f^{\prime}$ of dispatcher $r^{\prime} s^{\prime}{ }_{q}^{\prime}$ on the travel time of a vehicle type $f$ of dispatcher $r s_{q}$ is computed in constraints (17), (18) and (19). Constraints (17) and (18) are mathematical forms of if-then conditions and determine the delay of vehicles in waiting links based on the FIFO discipline (i.e. the vehicles that arrive at the starting node of a waiting link earlier leave the link earlier). Depending on traveling or not traveling through a waiting link by a vehicle type $f$ of dispatcher $r s_{q}$ and a vehicle type $f^{\prime}$ of dispatcher $r^{\prime} s^{\prime} q^{\prime}$ and when the vehicles arrive at the starting node of the waiting link, eight possible scenarios may occur. In Appendix C, it is proved that all the scenarios are considered by constraints (17), (18) and (19).

The possibility of using vehicle type $f$ to ship the hazmat cargos of dispatcher $r s_{q}$ depends on the number of vehicle type $f$ available at the origin node of the dispatcher. Constraint (20) calculates the number of vehicles of type $f$ available at the beginning of each period for dispatcher $r s_{q}$ by subtracting the number of vehicles departing the origin in the previous periods from the total number of the vehicles $\left(F^{r s_{q f}}\right)$.

Inner level conversion by Karush-Kuhn-Tucker (KKT): To convert the proposed bi-level model to a single level, the first-order KKT conditions are applied to the inner subproblem. By substituting (22) in (21) and calculating $d P / d p_{l^{t}{ }_{m}}$, the expected payoff of demon $m$ is as follows:

$$
\theta_{l^{t} m}=\sum_{l^{t}{ }_{1}=1}^{\left|A^{\prime}\right|} \ldots \sum_{l^{t}{ }_{k}=1, k \neq m}^{\mid A^{\prime}} \cdots \sum_{l^{t}{ }_{M}=1}^{\left|A^{\prime}\right|} \times\left\{\left(\prod_{k=1, k \neq m}^{M} p_{l^{t}{ }_{k}}\right)\left[\sum_{a^{t}} C_{a^{t},\left(l^{t}{ }_{1}, \ldots, l^{t}{ }_{m}, \ldots, l^{t}{ }_{M}\right)}(V)\right]\right\}
$$

According to (22) and (23), condition (28) is equivalent to (29) and (30). Condition (29) calculates the probability that link $a^{t}$ would be selected by at least one demon excluding demon $m$. These probabilities are needed 
in condition (30).

$$
\begin{aligned}
& x_{a^{t},-m}=1-\prod_{k=1, k \neq m}^{M}\left(1-p_{l^{t}{ }_{k}}\right) \\
& \theta_{l^{t} m}=C_{l^{t} m}(V)+\sum_{a^{t} \neq l^{t} m}^{\left|A^{\prime}\right|} x_{a^{t},-m} C_{a^{t}}(V)
\end{aligned}
$$

Each demon aims to maximize its expected payoff as follows:

$$
\pi^{m} \underset{l^{t}}{m} \max \theta_{l^{t} m}
$$

Therefore, applying the first-order KKT conditions to the demon subproblem results in the following nonlinear complementarity conditions:

$$
\begin{array}{ll}
\text { (3), (4), (5), (24), (25), (29), (30), and } & \\
p_{l^{t}}\left\{\pi^{m}-\theta_{l^{t} m}\right\}=0 & \forall m=1, \ldots, M, l^{t} m=1, \ldots,\left|A^{\prime}\right| \\
\pi^{m}-\theta_{l^{t} m} \geq 0 & \forall m=1, \ldots, M, l^{t} m=1, \ldots,\left|A^{\prime}\right|
\end{array}
$$

When $p_{l^{t}} \geq 0$, condition (32) ensures that $\pi^{m}-\theta_{l^{t} m}$ is equal to zero. This means the expected payoff of demon $m$ is the maximum expected payoff that can be achieved by demon $m$. Conversely, if the expected payoff of a link is less than the maximum achievable value $\left(\pi^{m}-\theta_{l^{t} m} \geq 0\right)$, then $p_{l^{t}}=0$. Inequality (33) imposes the condition that if demon $m$ does not assign an incident probability to link $l_{m}^{t}\left(p_{l^{t}}{ }_{m}=0\right)$, then its expected payoff must be less than the maximum achievable payoff.

Figure 4 presents the structure of the bi-level programming used to model the Stackelberg game after applying KKT on the demon subproblem. Note that there are two objective functions for the leader and just one is involved in the game with the follower which is provided by a set of complementary conditions.

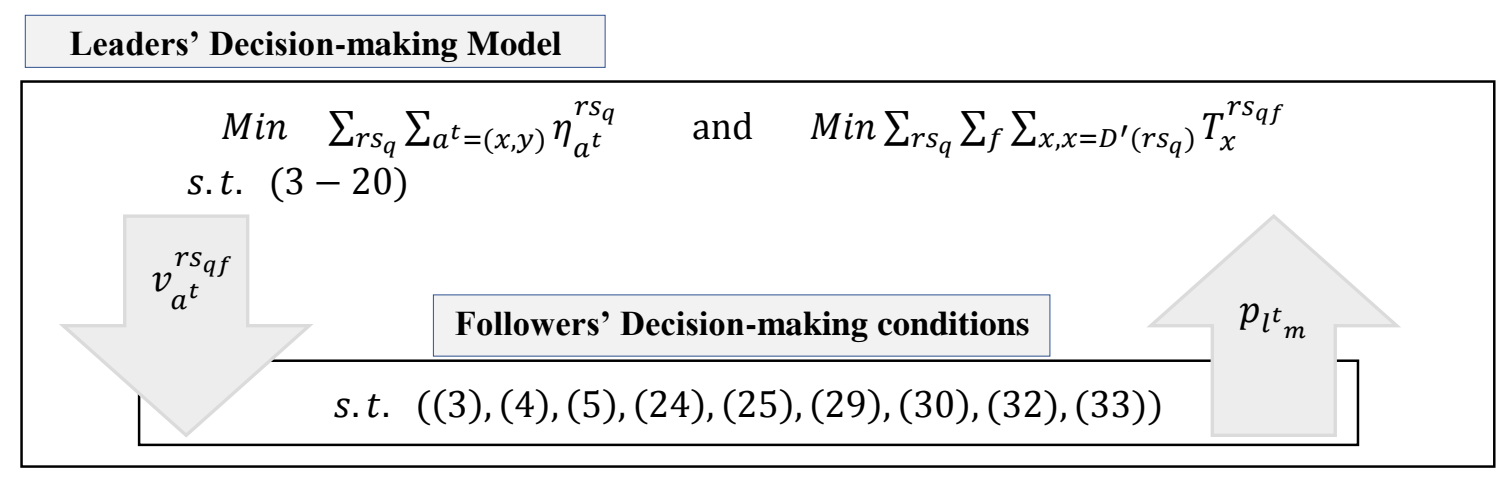

Figure 4. Structure of the bi-level programming.

It is noted that the current model has the ability to impose restrictions on movements through links and nodes. We can apply these restrictions either implicitly (by considering long travel times in some periods so that dispatchers avoid them at that period) or explicitly (by directly closing a link in a specific period when entering the data).

The proposed model is a mixed integer nonlinear bi-objective problem, which has $\left|A^{\prime}\right|$ links, $M$ demons, $|B|$ vehicle types and $H$ dispatchers. The main decision variables are $p_{l_{m}^{t}}, v_{a^{t}}^{r s_{q f}}$ and $Z_{a^{t}}^{r s_{q f}}$. The numbers of decision variables $p_{l^{t}{ }_{m}}$ and $v_{a^{t}}^{r s_{q f}}$ in a network are equal to $\left|A^{\prime}\right| \times M$ and $\left|A^{\prime}\right| \times|B| \times H$, respectively. The binary decision variables are $Z_{a^{t}}^{r s_{q f}}$ that depend on the values of $p_{l_{m}^{t}}$ and $v_{a^{t}}^{r s_{q f}}$. The numbers of these decision variables in the problem are $\left|A^{\prime}\right| \times|B| \times H$. Such a problem with a real-life network size on a commercial optimization software product cannot be solved in a reasonable time. Note that hazmat routing-scheduling is not a strategic decision-making problem; it is a tactical/ operational decision. In reality, this can be a day-to-day plan in which planners do not have much time to wait for a solver to get the optimal answer. Even if there is ample time, the complexity of the problem and the run time grow exponentially, which is not sufficient for real problems. We have verified the correctness of the proposed model by applying and visualizing it on several sample networks. Refer to Appendix D to see one of them (sample problem A). To get the results of the model for these sample problems, the commercial software 
products of GAMS and ALPHAECP have been used.

\section{SOLUTION APPROACH}

Our designed mathematical model is a mixed-integer, non-linear, bi-objective optimization model. Multi-objective optimization (MOO) models consist of several conflicting objective functions. Therefore, for MOO models, we can find a set of Pareto optimal (non-dominated) solutions instead of a unique optimal solution. In Pareto optimal solutions, an objective function cannot be improved without detriment to the other objective functions. For more information about the MOO methods, interested readers may refer to Cohon and Marks (1975), Hwang and Masud (1979), Ehrgott (2005), and Greco et al. (2016). In the proposed bi-level, bi-objective programming model (Figure 4 ), we use the $\varepsilon$-constraint method to deal with the objective functions. The $\varepsilon$-constraint method optimizes one of the objective functions and converts others to constraints (Chankong and Haimes, 2008; Cohon, 2004). For more information about this approach, interested readers may refer to Mavrotas (2007), Roman and Rosehart (2006) and Mavrotas (2009). To solve our model, we use the AUGMECON method, which is a version of the $\varepsilon$-constraint method incorporating lexicographic optimization with a payoff table (Mavrotas, 2009). In this regard, we optimize the risk objective and convert the travel time objective to a constraint. The structure and details of the AUGMECON method are explained in Appendix E.

As we noted at the end of section 4, the proposed model has $\left|A^{\prime}\right| \times M+\left|A^{\prime}\right| \times|B| \times H$ continuous decision variables and $\left|A^{\prime}\right| \times|B| \times H$ binary decision variables. This model is solvable in a rational computational time only for small- and medium-scale networks using commercial optimization software such as GAMS (with ALPHAECP solver). But for real-size and large-scale networks such as the road network of Singapore, we need an efficient and swift solution algorithm. For instance, if we consider the road network of Singapore, which has 22 nodes and 29 links (considering the approved hazmat routes by the government), and suppose we have 12 time-periods, 7 waiting links, 3 vehicle types, 2 demons and 2 dispatchers, then the number of continuous and binary decision variables are equal to 3784 and 2808, respectively. Accordingly, to solve this real-size and large-scale network, a solution approach is developed in subsection 5.1 .

\subsection{The ALNS Algorithm}

In this section, we develop a modified metaheuristic algorithm to solve the proposed model for large-scale networks. For routing and scheduling problems, Large Neighborhood Search (LNS) is recognized as the most successful metaheuristic method (Pisinger and Ropke, 2010). ALNS is an extension of LNS that is widely used in the literature to solve routing/ scheduling models (Azi et al., 2014; Demir et al., 2012; Grangier et al., 2016; Kovacs et al., 2012; Ribeiro and Laporte, 2012; Mohri et al., 2018). An ALNS algorithm is based on improving the current solution with some removal and insertion moves, iteratively. In each iteration, a removal and an insertion move are selected to change the current solution and produce a new solution. The process of selecting them is based on the weights of the moves. The weights of moves are calculated based on their performances in prior iterations through a random wheel mechanism. The detail of the process is explained in the following subsection titled "Computing the weight of moves." An ALNS algorithm is comprised of $N_{T}$ iterations and $n_{S}$ segments. The number of iterations in each segment is equal to $N_{T} / n_{S}$ (Ropke and Pisinger, 2006). The probability of the moves is updated at the beginning of each segment.

\section{The algorithm}

This modified metaheuristic approach includes six stages (Figure 5). The first three stages find an initial solution in 
the first iteration as well as new solutions for other iterations. Establishing these stages for producing a new solution is different from producing an initial solution.

Stage 1 -Generate a set of routes: In this stage, we produce a set of routes for each hazmat class $(q)$ and vehicle type $(f)$. The travel time of cargos depends on the hazmat class and the type of vehicle. Accordingly, we can generate a set of routes for each $r s_{q f}$. A Dijkstra algorithm (Dijkstra, 1959) with some modifications is used to produce a set of routes for each $r s_{q f}$. Since the model objectives are concerned about (i) total travel time as well as (ii) total risk, a new cost function is considered for network links to cover both objectives. One way to reduce loss in a network is by shipping the hazmat cargos using a mixed-route strategy. This strategy recommends using a mixture of routes for shipping hazmat cargos between an OD pair instead of taking a single route (Bell, 2006). However, a mixed-route strategy results in higher total travel time than a single route one. Therefore, to fulfill the concerns of both objectives, we consider the cost of a link to be proportional (or equal) to its travel time. We apply a penalty to the cost based on the usage frequency of the links in the previously generated routes. In this stage, we repeatedly update the penalty of the links based on their usage frequency in the previously generated routes. This approach intends not only to generate short routes but also to use a diverse set of links in generating routes to increase discrepancy. The size of the set generated for each $r s_{q f}$ is a random number between 1 and $d^{r s_{q}} / w_{f}$. Having a set with the size of $d^{r s_{q}} / w_{f}$ means there is a possibility to ship all vehicles of $r s_{q f}$ with different routes. There exist waiting links in STEN; the travel time of a waiting link is equal to the length of a time-period. Therefore, if a route passes through a waiting link, total accumulated costs (time $\times$ frequency) of the crossed link in the current period must be eliminated and only the cost of the waiting link (length of time-period $\times$ frequency of the waiting link) is added. Accordingly, we have modified our Dijkstra algorithm to embed this limitation.

Only an initial set of routes is generated for each $r s_{q}$ and each vehicle type $f$ in the first iteration of the first segment. For the next iterations, if the algorithm visits this stage, a link is selected based on removal moves 1 (the detail will be explained in subsection 5.2) and put in an exclusion set, which is specialized for the dispatcher and the vehicle type. As a result, for the iteration, the shortest path algorithm does not allow using the links which are in the exclusion set. We let the shortest path algorithm build the route sets without any insertion moves because we never receive any insignificant improvement with assuming some intentional insertion moves into the algorithm. 
Considering an equal probability for return loops and moves of stages

$i=1$ (a counter for segment' iterations) and $j=1$ (a counter for problem' segments)

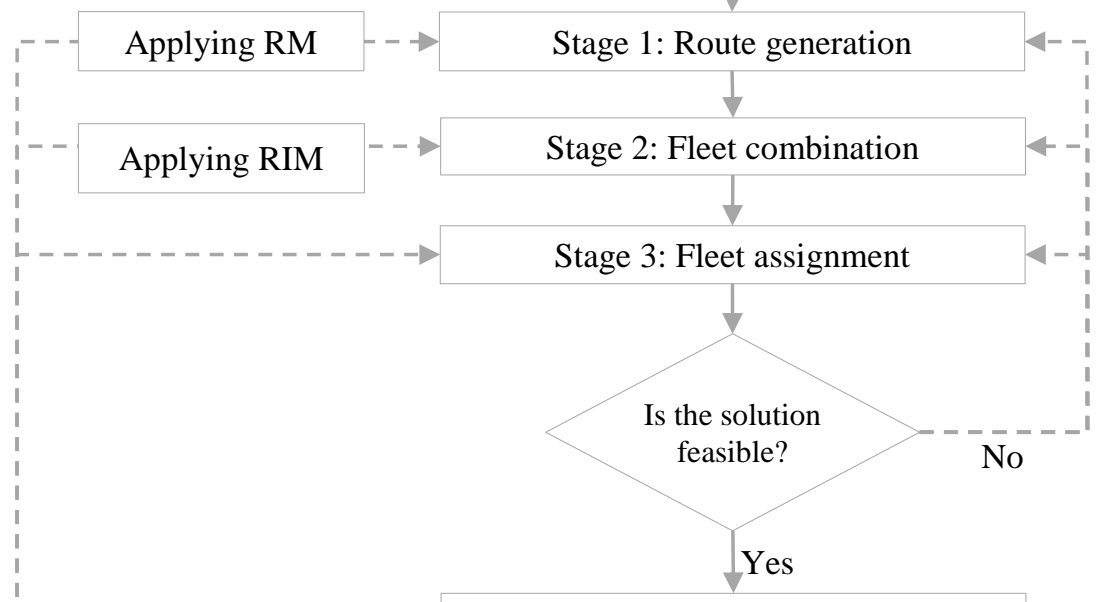

Stage 4: Objectives computation

Stage 5: Investigating the acceptance criteria and updating the current solution and the set of non-dominated solutions by the modified SA algorithm

Updating the score of return loops and stages' moves

Finding the return loops with a roulette wheel mechanism

Updating the weights and probabilities of return loops and moves of the stages

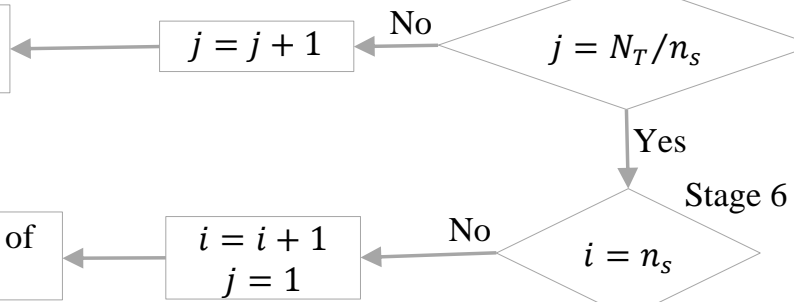

Yes

End

Figure 5. The flowchart of the modified metaheuristic approach.

Stage 2 -Determine combinations of the fleet: For shipping the cargoes of each $r s_{q}$, a subset of vehicles is needed. The feasibility of each vehicle subset depends on the number of vehicles available in the depots and limitations force by constraints (21) and (22). For each dispatcher $r s_{q}$, the number of vehicles type $f$ existing in the subset must be less than or equal to $F^{r s_{q f}}$. Constraints (21) and (22) ensure that the total capacity of vehicles in the subset is greater than or equal to the total demand of dispatcher $r s_{q}$. If this stage is used for improving a current solution and finding a new solution, the removal and insertion moves of Stage 2 (RIM) (which will be explained in subsection 5.2), are used. Otherwise, a random mechanism is applied to finding the initial solution.

Stage 3 - Assign fleet combinations to the routes: In this stage, the vehicles' combination determined in stage 2 is assigned to the routes generated in stage 1 randomly. Then, the feasibility of assignments is checked based on constraint (33) because some routes have one or more waiting links. Assigning a vehicle type $f$ to a route in period $t$ and having a waiting link decreases one unit of the available vehicle type $f$ for the next time-period $(t+1)$. The reason is that when time-period $t+1$ is started, the vehicle is still en-route. In the mathematical model, constraint (33) ensures the availability of vehicles in each time-period; therefore, we exploit it to check the feasibility of the assignment. If the assignment is feasible, go to stage 4; otherwise, return to stages 3, 2 and 1, respectively. Each 
returning stage is repeated $k$ times ( $k$ is set to a random number between 5 and 10) and if the assignment is still unfeasible, the returning stage changes to its upper stage. The selection of the returning stage in stage 6 will be described in subsection 5.2.

Stage 4 -Calculate the objective functions: In this stage, the FIFO discipline is applied to the feasible assignments of stage 3 and the value of the second objective (travel time) is calculated. Then, the maximum payoff (first objective named risk) of the assignment is calculated using the demons' subproblem.

Stage 5 - Update the set of Pareto optimal solutions: In this stage, if the assignment found in Stage 4 is a Pareto optimal solution, it is added to the set of Pareto (non-dominated) solutions.

Stage 6 - Check the termination condition: In this stage, the termination condition is checked. If it is valid, terminate the algorithm and report the set of Pareto optimal solutions; otherwise, return to stage 1, 2 or 3 . In subsection 5.2 , the termination condition of stage 6 is discussed.

\section{Innovations in the traditional ALNS}

In a traditional ALNS, in each iteration, removal and insertion processes are being performed, repetitively, to destroy an existing current solution and repairing it to generate a new solution, hoping for some improvement in the objective function values. This process can cause some issues to our hazmat routing-scheduling model:

- There is a possibility that the nominated removal and insertion moves work on two different partitioning decisions. In this regard, the solution algorithm will either fail or take a long time to investigate the destroyed parts of the solution and repair all of them. To resolve this issue, we propose a modified ALNS that categorizes removal and insertion moves into some subsets based on the decisions that they make. Accordingly, if a removal move is selected from subset $A$, then an insertion move must be selected from this subset.

- The presented model in this study is comprised of three partitioning decision variables, namely: route selection, vehicle type selection, and vehicle assignment to routes. Therefore, we consider three subsets for removal and insertion moves. Therefore, in each iteration, initially, a subset is selected with a random wheel using the weights of all the subsets obtained from the previous iterations. Then, removal and insertion moves are chosen from the moves of the selected subset.

- Conventionally, an acceptance criterion in an ALNS algorithm with an SA local search is based on comparing a current, a new, and the best solution (Mohri et al., 2018), while we have a bi-objective model looking for a set of non-dominated solutions instead of one best solution. Therefore, we made some changes in the process of investigating the acceptance criteria to produce a set of non-dominated solutions instead of a single best solution in the end.

\subsection{Detail of Each Stage in the ALNS Algorithm}

In the previous subsection, the ALNS algorithm was described succinctly. Some stages need more elaboration, and they are explained in this subsection.

\section{The removal moves of Stage $1(\mathrm{RM})$}

We have three different removal moves in stage $1(\mathrm{RM})$ of the metaheuristic approach, which are explained below. Through these moves, a link is selected in each iteration to be assigned to the set of excluded links. Links of this set are not to be selected in stage 1. In each iteration, one of these moves is selected to improve the current solution. The selection mechanism of these moves is also explained in this subsection. These moves are as follows:

Random removal $(R R)$ : This move selects a link from the links of the selected routes of each dispatcher and puts 
it into the set of excluded links. The links of this set are removed from the selection in stage 1 . For each $r s_{q}$, a random link is excluded by this move in each iteration.

Time-based removal (TBR): Firstly, this move calculates the share of each link in the amount of travel time objective. Then, the shares of the links are normalized by dividing their values by the maximum share. The weight of each link is calculated by dividing its normalized share with the summation of the normalized shares of all links. Finally, by using a roulette wheel mechanism for the links' weights, a link is selected for each $r s_{q}$ and put it into the set of excluded links.

Risk-based removal (RBR): This move is similar to TBR but the links' shares are calculated using the risk instead of the travel time.

\section{The removal and insertion moves of Stage 2 (RIM)}

In this stage, three concurrent removal moves in stage 2 (RIM) are proposed to remove some vehicle types from the fleet combination and add some other vehicle types to it. Each RIM follows a specific goal and its removal and insertion are adjusted based on this:

Random removal and insertion (RRI): This operator makes a new possible fleet combination for each dispatcher randomly. Accordingly, stage 3 in subsection 5.1 is performed repeatedly until a different fleet combination is obtained.

Small/ large fleet removal/insertion (SLFRI): Using small vehicles increases the travel time objective but decreases the risk objective in the network. In these removal and insertion moves, a new fleet combination with fewer total vehicles than the current solution is proposed randomly. Accordingly, in stage 3 of subsection 5.1 the number of selected vehicles in new the fleet combination is bound to be less than the number of vehicles in the current solution.

Large/ small fleet removal/insertion (LSFRI): Unlike SLTRI, using large vehicles reduces the travel time objective in the network but increases the risk objective in the network. This move produces a new fleet combination with more vehicles used than the current fleet combination randomly. In this regard, the total number of vehicles in the new fleet combination is assumed to be more than the total vehicles in the current solution. This assumption is applied to stage 3 of subsection 5.1 .

\section{Acceptance criteria}

In ALNS, the substitution of the current solution with a new one is done according to some acceptance criteria. The acceptance criteria should evaluate the superiority of the new solutions compared to the current ones while avoiding local minimums. We have modified the acceptance criteria proposed by the SA algorithm to adjust it for the biobjective model. The traditional acceptance criteria presented by the SA algorithm for single-level models are based on comparing the new and the current solutions. If one of the acceptance criteria is satisfied, the current solution is replaced with the new one. The current solution always exists in the memory of the algorithm. In our approach, the algorithm considers the current solution as well as a set of non-dominated solutions in its memory. Therefore, the acceptance criteria are defined based on comparing the current solution, the new solution and the set of nondominated solutions as follows:

(1) The new solution dominates the current one (or the current solution cannot dominate the new solution) and the new solution is not dominated by the set of non-dominated solutions. In this situation, the new solution is accepted without further considerations because it may improve the solution of the problem in the next iterations. Therefore, the current solution is substituted with the new one. Moreover, the new solution is added to the non- 
dominated set of solutions and the set is updated based on excluding the dominated ones.

(2) The new solution dominates the current one but it is dominated by the set of non-dominated solutions. In this situation, the new solution is accepted without further considerations because it may improve the solution of the problem in the next iterations. The current solution is substituted with the new one.

(3) The new solution cannot dominate the current one and it is dominated by the set of non-dominated solutions. Therefore, the new solution is accepted with the probability of $e^{-\left(\left(f_{1}\left(s_{\text {new }}\right)+f_{2}\left(s_{\text {new }}\right)\right)-\left(f_{1}\left(s_{\text {current }}\right)+f_{2}\left(s_{\text {current }}\right)\right)\right) / T}$. Accordingly, the current solution is substituted with the new one if the new one is accepted. $s_{\text {new }}$ and $s_{\text {current }}$ represent the new and current solutions, $f_{1}$ and $f_{2}$ show the first and second objective functions and $T$ is the temperature. In the first iteration, the temperature is set equal to $\left(f_{1}\left(s_{\text {initial }}\right)+f_{2}\left(s_{\text {initial }}\right)\right) \times C_{\text {initial }}$, where $s_{\text {initial }}$ and $0<C_{\text {initial }}<1$ representing the initial solution and an initial constant, respectively. At the end of each iteration, the temperature is updated by substituting $h T$ with $T$, where $h$ is a constant number between zero and one.

\section{Termination criterion}

The ALNS algorithm is comprised of $n_{s}$ segments that each segment includes some iterations. Accordingly, the algorithm ends after a fixed number of segments.

\section{Computing the weight of returning decisions}

In stage 5, the algorithm returns to previous stages to improve the current solution. This return may happen in three different ways:

Return to stage 1: The algorithm returns to stage 1 to create some new routes. Then, it goes through stages 2 to 6 to create a new solution.

Return to stage 2: The algorithm returns to stage 2 to create a new fleet combination. Then, it goes through stages 3 to 6 to create a new solution.

Return to stage 3: The algorithm returns to stage 3 to assign generated routes to fleet combinations differently. Then, it goes through stages 4 to 6 to create a new solution.

An ALNS algorithm is comprised of $N_{T}$ iterations and $n_{S}$ segments. The number of iterations in each segment is equal to $N_{T} / n_{S}$ (Ropke and Pisinger, 2006). Choosing one of the return decisions for an iteration is performed based on the weights of returning decisions computed during past segments. In each segment, the weights of these returns are updated according to their performance in prior segments and they are fixed along the segment. Initially, for iterations of the first segment, all returns have equal weights. The weights of these returns for the next segments are calculated as follows:

$$
W_{r}^{s}=W_{r}^{s-1}(1-\alpha)+\alpha \frac{s_{r}^{s-1}}{N_{r}^{s-1}} \quad \forall s, r
$$

Where $\alpha$ is a constant reaction factor, $N_{r}^{s-1}$ is the number of times that return $r$ was used in the last segment $(s-1)$, and $s_{r}^{s-1}$ is the score of return loop $r$ in the last segment $(s-1)$, respectively.

Constraint (34) demonstrates the process of updating the weight of a return for each segment. The weight of a return loop $(r)$ for all iterations of segment $s\left(W_{r}^{s}\right)$ is computed based on its weight for prior segment $s-1\left(W_{r}^{s-1}\right)$ and its score in segment $s-1\left(s_{r}^{s-1}\right)$. At the beginning of a segment, the score of a return is zero and is updated during the segment's iterations based on the acceptance situations (discussed in this section), which have occurred for the segment's iterations using the return. Accordingly, if situations 1, 2 or 3 occur for a new solution in an iteration of segment $-1, s_{r}^{s-1}$ is updated by adding $\sigma_{1}, \sigma_{2}$ and $\sigma_{3}$ values to it. Constraint (35) computes the selection 
probability of return $r$ in segment $s\left(P_{r}^{S}\right)$ based on returns' weights. Based on the probabilities of returns and a roulette wheel mechanism, one of the returns will be selected for an iteration in segment $s$.

$$
P_{r}^{s}=\frac{W_{r}^{s}}{\sum_{r} W_{r}^{s}} \quad \forall s, r
$$

\section{Computing the weight of moves}

The process of computing the weight and probability of moves in each stage is similar to the process of computing the weight and probability of return loops (constraints (34) and (35)). To consider these constraints, some modifications are required as follows: (i) index $r$ refers to the removal or insertion move instead of the return loop; (ii) instead of $\sigma_{1}, \sigma_{2}$ and $\sigma_{3}$ for updating the score of returns, $\sigma_{1}^{\prime}, \sigma_{2}^{\prime}$ and $\sigma_{3}^{\prime}$ are used for updating the score of removal or insertion moves.

\section{PARAMETER SETTING AND VALIDATION}

This section is comprised of three subsections, namely system specifications, parameter tuning, and validation. In the subsection system specifications, the features of the device which was used to solve the model with two exact and metaheuristic approaches are explained. In subsection parameter tuning, the best obtained values for the parameters in the metaheuristic approach as well as the process taken to produce them, are discussed. Finally, in the last subsection, the manner of producing some test problems in addition to a comparison between the results of the exact and metaheuristic approaches are explained.

\subsection{System Specifications}

To solve the model with an exact approach, the commercial software products of GAMS with solver ALPHAECP are used. To solve the model by the proposed solution approach, the commercial software MATLAB is used. The codes, both exact and metaheuristic codes, were run on a system with 4 GB RAM, 7 core CPU and 4 MB cache.

\subsection{Parameter Tuning}

An initial analysis was established on the ALNS and SA parameters to fine-tune them and this led to some typical values as shown in column 4 of Table 2. In this regard, we considered the exact solutions of the presented sample problem A as reference solutions for parameter tuning. Table 2 shows the existing parameters in the model, their choice sets and the final typical values which resulted. Although the typical values work well not only for sample problem A but also for other test networks reported in the validation subsection, we cannot claim these are the best possible.

To do the parameter tuning analysis, we initially considered a set of choices for each parameter, illustrated in column 3 of Table 2. According to the amount of choice for each parameter, in total $4 \times 3^{8}$ scenarios were defined for setting up the ALNS and SA parameters, shown in column 1 of Table 2. Then, the metaheuristic approach was solved for all the scenarios and the gaps between minimum travel time of the ALNS and GAMS (Gap1) and minimums risk of the ALNS and GAMS (Gap2) were calculated. After that, the gaps were normalized by dividing them by the minimum objective values of GAMS, respectively. Next, the minimum of the normalized values of Gap1 and Gap2 was computed and named Min-Gap. Finally, the parameter values of the scenario which has the minimum Min-Gap are reported as the typical parameter values, shown in column 4 of Table 2. Figure 6 shows the results of two solution methods on sample problem A based on the typical values used in the ALNS algorithm. 
Table 2: Metaheuristic parameters, their choice sets and their final typical values.

\begin{tabular}{|c|c|c|c|}
\hline Parameter & Parameter description & Choice set & $\begin{array}{l}\text { Typical } \\
\text { value }\end{array}$ \\
\hline$N_{T}$ & Total iterations of the ALNS algorithm & $\{1000,2000,3000\}$ & 3000 \\
\hline$n_{s}$ & Total iterations in segment $s$ of the ALNS algorithm & $\{15,30\}$ & 15 \\
\hline$\alpha$ & Constant reaction factor between zero and one & $\{0.1,0.3\}$ & 0.1 \\
\hline$C_{\text {initial }}$ & A constant number between zero and one used in SA local search & $\{0.0001,0.00015,0.0002\}$ & 0.0001 \\
\hline$h$ & $\begin{array}{l}\text { A constant number between zero and one used in SA local search } \\
\text { (cooling rate) }\end{array}$ & $\{0.999\}$ & 0.999 \\
\hline$T$ & $\begin{array}{l}\text { The temperature used in SA local search }\left(T=\left(f_{1}\left(s_{\text {initial }}\right)+\right.\right. \\
\left.\left.\qquad f_{2}\left(s_{\text {initial }}\right)\right) \times C_{\text {initial }}\right)\end{array}$ & Depends to $C_{\text {initial }}$ & 80 \\
\hline$\sigma_{1}$ & $\begin{array}{l}\text { The amount of value added to a return loop if situation } 1 \text { (discussed in } \\
\text { the subsection titled "Acceptance and termination criteria") occurred }\end{array}$ & $\{1,3,5\}$ & 3 \\
\hline$\sigma_{2}$ & $\begin{array}{l}\text { The amount of value added to a return loop if situation } 2 \text { (discussed in } \\
\text { the subsection titled "Acceptance and termination criteria") occurred }\end{array}$ & $\{1,3,5\}$ & 1 \\
\hline$\sigma_{3}$ & $\begin{array}{l}\text { The amount of value added to a return loop if situation } 3 \text { (discussed in } \\
\text { the subsection titled "Acceptance and termination criteria") occurred }\end{array}$ & $\{1,3,5\}$ & 5 \\
\hline$\sigma_{1}^{\prime}$ & $\begin{array}{l}\text { The amount of value added to a move score if situation } 1 \text { (discussed in } \\
\text { the subsection titled "Acceptance and termination criteria") occurred }\end{array}$ & $\{1,3,5\}$ & 3 \\
\hline$\sigma_{2}^{\prime}$ & $\begin{array}{l}\text { The amount of value added to a move score if situation } 2 \text { (discussed in } \\
\text { the subsection titled "Acceptance and termination criteria") occurred }\end{array}$ & $\{1,3,5\}$ & 1 \\
\hline$\sigma_{3}^{\prime}$ & $\begin{array}{l}\text { The amount of value added to a move score if situation } 3 \text { (discussed in } \\
\text { the subsection titled "Acceptance and termination criteria") occurred }\end{array}$ & $\{1,3,5\}$ & 3 \\
\hline
\end{tabular}

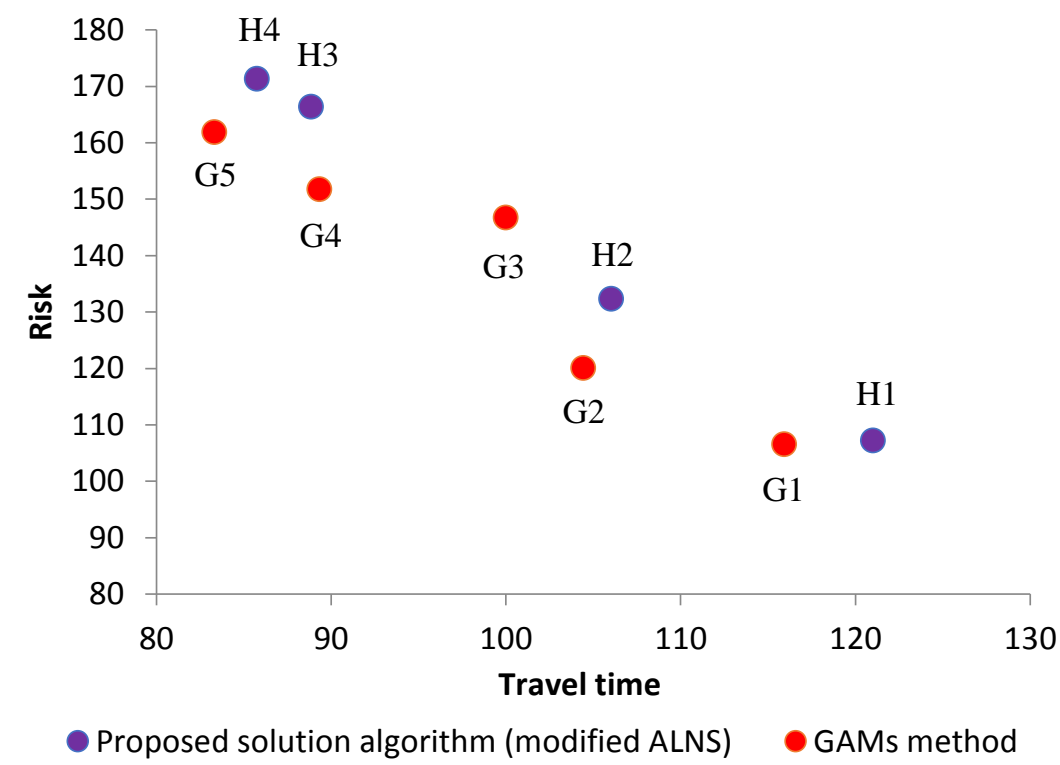

Figure 6. A comparison between the results of GAMS solver and the presented metaheuristic approach.

\subsection{Validation}

In this subsection, 14 types of test problems in different sizes (small, medium, and large) are generated, which are different in terms of the numbers of periods, nodes, links, hazmat classes, vehicle types and demons. Each type of test problem is run 10 times. Therefore, overall, 140 runs have been performed. To measure the quality of the objective functions and also the run time of the coded ALNS algorithm, they are evaluated versus the mathematical formulation coded on the GAMS solver. Note that the hazmat routing-scheduling problem is at the level of tactical/ operational planning, where we lack decision-making time in reality, particularly for day-to-day planning. Therefore, we allow the coded model and algorithm search for optimal solutions to be up to one hour. Table 3 shows the detailed specifications of test problems (e.g., the numbers of periods, nodes, links, etc.), the number of Pareto solutions, GAMS run time (seconds), the ALNS run time (seconds), maximum error (\%) and average error (\%).

To compare the run time of the GAMS solver and the proposed ALNS algorithm, we set the same numbers of 
non-dominated solutions for both solution types. To do this, first, we solved the test problem with the ALNS algorithm and obtained the non-dominated solutions. Then, we solved the test problem again with GAMS so that the number of non-dominated solutions was equal to that of the ALNS algorithm. In Table 3, the two last columns represent the maximum and the average percentage of errors between solutions of GAMS and the ALNS. Since the proposed model is a bi-objective, we took some steps to calculate a two-dimensional error measure. First, we connected the non-dominated solutions of the GAMS solver to each other to form the "Pareto front" for exact solutions. Next, we depicted the non-dominated solutions of the ALNS algorithm on the Pareto front to find an exact equivalent solution for each solution. Then, for each non-dominated solution of the ALNS algorithm, we computed the percentage of errors for first and second objectives for the non-dominated solution of the ALNS and their equivalent GAMS solution. The maximum and average percentage of errors are reported in Table 3.

In terms of runtime, it is evident that the ALNS is far faster than the GAMS solver. The largest test problem can be solved by the ALNS in 51.06 seconds while GAMS cannot solve test problems 10 through 14 in one hour. When it comes to the quality of the objective functions, for test problems that can be solvable by both GAMS and the ALNS, the maximum error is $4.2 \%$ and its average is $2.6 \%$. Interestingly, for small test problems, the ALNS obtains optimal solutions with no error.

Table 3. The input features and output results of solving test problems with GAMS and the ALNS.

\begin{tabular}{|c|c|c|c|c|c|c|c|c|c|c|c|c|c|c|c|}
\hline 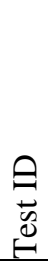 & $\begin{array}{l}\frac{0}{0} \\
\stackrel{0}{0} \\
0 \\
0\end{array}$ & $\begin{array}{l}\tilde{\theta} \\
\frac{0}{0} \\
z\end{array}$ & $\underset{\nexists}{\stackrel{\mathscr{J}}{\Xi}}$ & 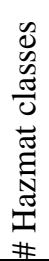 & $\begin{array}{l}0 \\
0 \\
2 \\
0 \\
0 \\
: 0 \\
0 \\
0 \\
\#\end{array}$ & 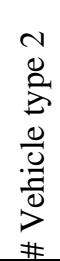 & $\begin{array}{l}m \\
0 \\
0 \\
0 \\
0 \\
0 \\
: 0 \\
0 \\
0 \\
\# \\
\#\end{array}$ & $\begin{array}{l}\mathscr{0} \\
\stackrel{0}{0} \\
\stackrel{0}{0} \\
\text { D }\end{array}$ & 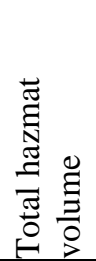 & 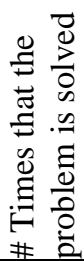 & $\begin{array}{l}0 \\
.0 \\
0 \\
0 \\
0 \\
0 \\
0 \\
0 \\
0 \\
0 \\
\# \\
\#\end{array}$ & 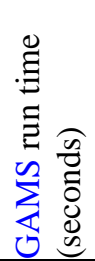 & 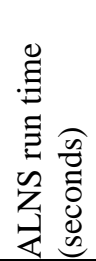 & 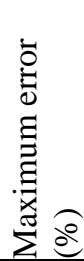 & 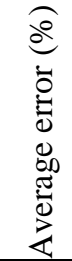 \\
\hline 1 & 2 & 8 & 9 & 1 & 4 & 0 & 2 & 1 & 40 & 10 & 2 & 75 & 0.005 & 0.00 & 0.00 \\
\hline 2 & 2 & 8 & 9 & 1 & 4 & 0 & 2 & 2 & 40 & 10 & 2 & 89 & 0.005 & 0.00 & 0.00 \\
\hline 3 & 2 & 8 & 9 & 1 & 8 & 0 & 4 & 2 & 80 & 10 & 2 & 586 & 0.005 & 0.00 & 0.00 \\
\hline 4 & 3 & 11 & 14 & 1 & 4 & 0 & 2 & 1 & 40 & 10 & 3 & 809 & 0.011 & 0.05 & 0.02 \\
\hline 5 & 3 & 11 & 14 & 1 & 4 & 0 & 2 & 2 & 40 & 10 & 3 & 1165 & 0.014 & 0.10 & 0.03 \\
\hline 6 & 3 & 11 & 14 & 1 & 8 & 0 & 4 & 2 & 80 & 10 & 3 & 213 & 0.029 & 0.00 & 0.00 \\
\hline 7 & 4 & 14 & 19 & 1 & 12 & 8 & 6 & 1 & 120 & 10 & 5 & 3961 & 0.022 & 0.00 & 0.00 \\
\hline 8 & 5 & 17 & 24 & 1 & 20 & 12 & 10 & 2 & 200 & 10 & 7 & 3401 & 0.041 & 1.20 & 0.80 \\
\hline 9 & 2 & 22 & 42 & 2 & 22 & 14 & 17 & 2 & 330 & 10 & 5 & 2897 & 0.231 & 4.20 & 2.60 \\
\hline 10 & 3 & 31 & 64 & 2 & 22 & 14 & 17 & 2 & 330 & 10 & 7 & - & 0.662 & - & - \\
\hline 11 & 4 & 40 & 86 & 2 & 40 & 24 & 20 & 2 & 500 & 10 & 8 & - & 13.76 & - & - \\
\hline 12 & 5 & 49 & 108 & 2 & 40 & 24 & 20 & 2 & 500 & 10 & 8 & - & 23.12 & - & - \\
\hline 13 & 6 & 58 & 130 & 2 & 80 & 60 & 40 & 2 & 1000 & 10 & 7 & - & 36.98 & - & - \\
\hline 14 & 7 & 67 & 152 & 2 & 150 & 100 & 80 & 2 & 2000 & 10 & 11 & - & 51.06 & - & - \\
\hline
\end{tabular}

\section{CASE STUDY}

We consider the road network of Singapore as the case problem (Meng et al., 2005; Szeto et al., 2017). The network consists of 22 nodes and 29 links (Figure 7). Trucks are not allowed to move in urban areas during dark times of a day. Therefore, the length of the time horizon is around 12 hours (e.g., from 7 am up to $7 \mathrm{pm}$ ). Note that Singapore is only $141 \mathrm{~km}$ from the equator. Therefore, this time span does not significantly change over the year. When it comes to traffic congestion, each day (12 hours) is divided into three time-periods: Period 1: 7:30-9:30 am; Period 2: 9:30 am-17:00 pm and Period 3:17:00-19:30 pm. This time split is in line with the dynamic tariff used in the freight and passenger transportation system in the country. The first and third periods include the morning and evening rush hours and are more congested; the second period is less congested. The model needs the average travel time of each link in each period. To make the model close to reality, we split a day into 12 time-periods with a length of one hour each. Transferring from one period to the next one is done through seven waiting links. Figure 7 shows the road network of Singapore and the location of its waiting links' origin nodes. 
Two different hazmat classes (liquid petroleum and flammable materials) are supposed to be shipped using this network. For each hazmat class, two OD pairs exist in the network. The flow unit is the liter and the capacity of tanker trucks range from 15000 to 26000 liters (Pradhananga et al., 2014, Seaco, 2018). We consider small, medium and large tanker trucks with a capacity of 15000, 20000, and 25000 liters, respectively. The OD pairs for the first hazmat class are from node 1 to 21 and from node 3 to 22 with 2 and 2.6 million liters, respectively. The OD pairs for the second hazmat class are 3 and 3.4 million liters from node 1 to 21 and from node 3 to node 22, respectively. The STEN for this network has 348 original links, 48 dummy links, and 77 waiting links. Two demons ${ }^{1}$ are assumed to attack the network's links during a day.

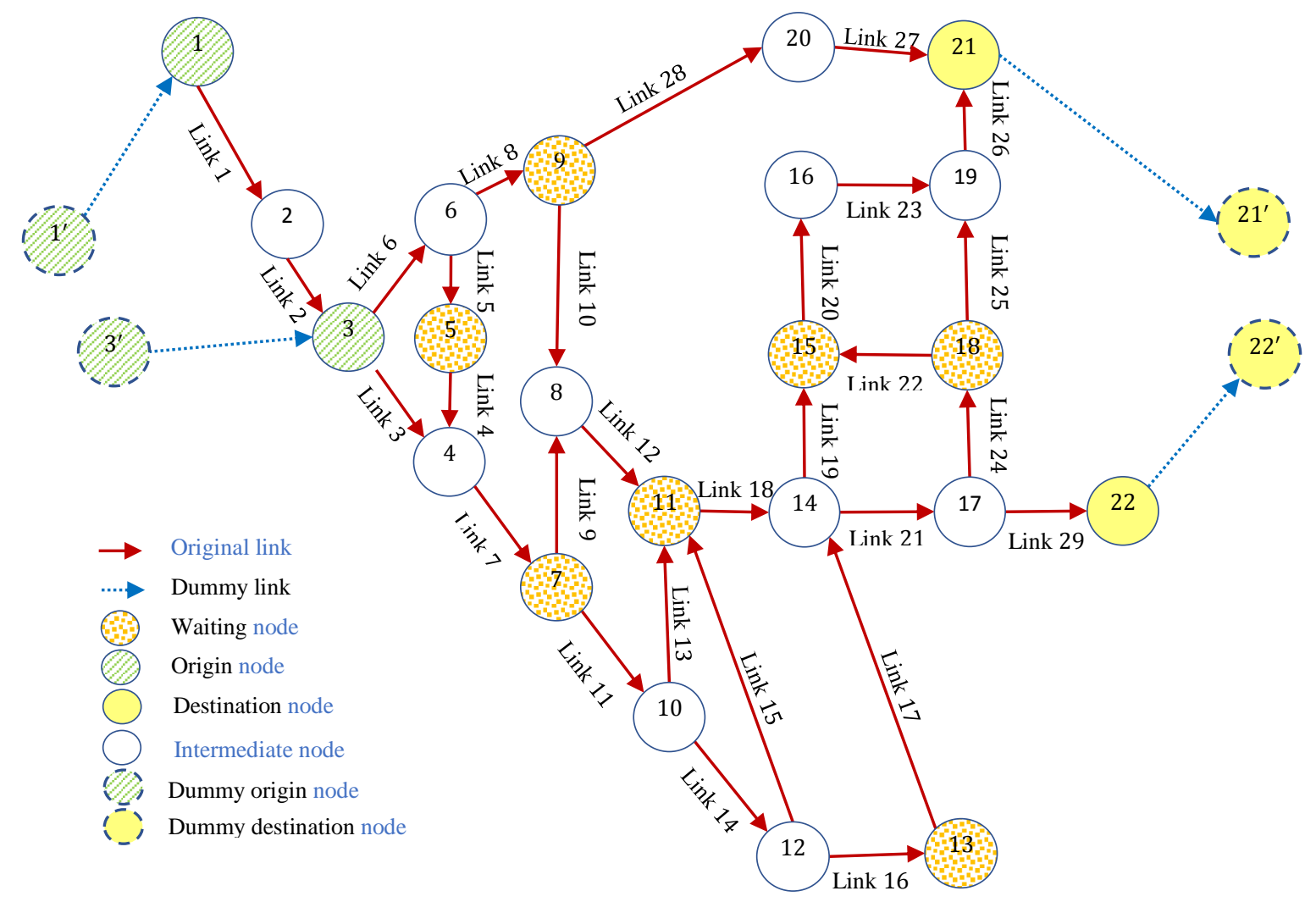

Figure 7. The original road network of Singapore.

The travel times of links for 12 time-periods, three vehicle types and two hazmat classes are estimated based on the detailed information presented in Meng et al. (2005) about the Singapore network. In Meng et al. (2005) the length, type, average speed of vehicles and their changes for network links are presented. We assume that smaller vehicles can move faster than the larger ones. Table 4 shows the length and type of network's links and Table 5 demonstrates the travel speed of each vehicle type on each link type and in each time-period. It is assumed that the type of carried hazmat class does not affect the travel speed of vehicles.

The radii of the impact zones around each link for hazmat classes 1 and 2 are 1600 and 800 meters, respectively (Szeto et al., 2017). The amount of loss for each link depends on the time-period, hazmat type and its volume and are calculated using parameters $\beta_{a^{t}}^{q f}$ and $\alpha_{a^{t}}^{q f}$ in constraint (14). In Szeto et al. (2017), the total loss of a link induced by the total volume of hazmat class $i$ was noted for two working and non-working periods. In this paper, we consider periods 1,11 , and 12 as a non-working period and the others as a working period. Accordingly, to avoid unnecessary complexity, we estimate parameters $\beta_{a^{t}}^{q f}$ for network links by assuming parameters $\alpha_{a^{t}}^{q f}$ to be equal to one. The total loss of a link caused by hazmat class $i$ happens when the whole amount of hazmat is placed on the link. The amount

${ }^{1}$ Apart from subsection 8.2, all the problems in this paper are solved for two demons. Note that, in subsection 8.2, we gradually increase the number of demons up to 5 to investigate its impact on tradeoff between the objectives. 
of loss caused by a unit of hazmat (e.g., one liter) is calculated by dividing it by the total loss over the whole amount of hazmat. Multiplying the capacity of a vehicle by the loss caused by a unit of hazmat yields $\beta_{a^{t}}^{q f}$ for the corresponding link and vehicle type.

Table 4. The length of the network's links.

\begin{tabular}{ccccccccc}
\hline Link & Length $(\mathrm{m})$ & Link type* & Link & Length $(\mathrm{m})$ & Link type & Link & Length $(\mathrm{m})$ & Link type \\
\hline $1 \rightarrow 2$ & 16323 & $\mathrm{~S}$ & $7 \rightarrow 10$ & 1243 & $\mathrm{E}$ & $14 \rightarrow 17$ & 915 & $\mathrm{P}$ \\
$2 \rightarrow 3$ & 1185 & $\mathrm{P}$ & $8 \rightarrow 11$ & 1830 & $\mathrm{P}$ & $18 \rightarrow 15$ & 930 & $\mathrm{P}$ \\
$3 \rightarrow 4$ & 1647 & $\mathrm{E}$ & $10 \rightarrow 11$ & 1220 & $\mathrm{P}$ & $16 \rightarrow 19$ & 1403 & $\mathrm{P}$ \\
$5 \rightarrow 4$ & 1708 & $\mathrm{~S}$ & $10 \rightarrow 12$ & 3919 & $\mathrm{E}$ & $17 \rightarrow 18$ & 1120 & $\mathrm{~S}$ \\
$6 \rightarrow 5$ & 1037 & $\mathrm{~S}$ & $12 \rightarrow 11$ & 4268 & $\mathrm{P}$ & $18 \rightarrow 19$ & 1708 & $\mathrm{~S}$ \\
$3 \rightarrow 6$ & 1243 & $\mathrm{P}$ & $12 \rightarrow 13$ & 1598 & $\mathrm{P}$ & $19 \rightarrow 21$ & 3528 & $\mathrm{~S}$ \\
$4 \rightarrow 7$ & 4895 & $\mathrm{E}$ & $13 \rightarrow 14$ & 5205 & $\mathrm{E}$ & $20 \rightarrow 21$ & 2379 & $\mathrm{P}$ \\
$6 \rightarrow 9$ & 4997 & $\mathrm{P}$ & $11 \rightarrow 14$ & 3026 & $\mathrm{P}$ & $9 \rightarrow 20$ & 7320 & $\mathrm{P}$ \\
$7 \rightarrow 8$ & 2071 & $\mathrm{E}$ & $14 \rightarrow 15$ & 1200 & $\mathrm{E}$ & $17 \rightarrow 22$ & 2649 & $\mathrm{P}$ \\
$9 \rightarrow 8$ & 7134 & $\mathrm{E}$ & $15 \rightarrow 16$ & 2135 & $\mathrm{E}$ & & & \\
\hline
\end{tabular}

*S: Secondary road; E: Expressway; P: Primary road

Table 5. The average travel speed of each vehicle type.

\begin{tabular}{|c|c|c|c|c|c|c|c|c|c|c|c|c|c|}
\hline \multirow{2}{*}{ Vehicle type } & \multirow{2}{*}{$\begin{array}{l}\text { Link } \\
\text { type* }\end{array}$} & \multicolumn{12}{|c|}{ The average speed for time-periods $(\mathrm{Km} / \mathrm{h})$} \\
\hline & & 1 & 2 & 3 & 4 & 5 & 6 & 7 & 8 & 9 & 10 & 11 & 12 \\
\hline \multirow{3}{*}{ Small } & $\mathrm{E}$ & 75 & 60 & 85 & 86 & 80 & 78 & 85 & 94 & 90 & 75 & 65 & 70 \\
\hline & $\mathrm{P}$ & 60 & 45 & 62 & 62 & 55 & 53 & 65 & 75 & 70 & 60 & 50 & 55 \\
\hline & $\mathrm{S}$ & 42 & 36 & 44 & 45 & 40 & 40 & 55 & 65 & 60 & 50 & 40 & 45 \\
\hline \multirow{3}{*}{ Medium } & $\mathrm{E}$ & 68 & 54 & 77 & 77 & 72 & 70 & 77 & 85 & 81 & 68 & 59 & 63 \\
\hline & $\mathrm{P}$ & 54 & 41 & 56 & 56 & 50 & 48 & 59 & 68 & 63 & 54 & 45 & 50 \\
\hline & S & 38 & 32 & 40 & 41 & 36 & 36 & 50 & 59 & 54 & 45 & 36 & 41 \\
\hline \multirow{3}{*}{ Large } & E & 65 & 52 & 73 & 74 & 69 & 67 & 73 & 81 & 77 & 65 & 56 & 60 \\
\hline & $\mathrm{P}$ & 52 & 39 & 53 & 53 & 47 & 46 & 56 & 65 & 60 & 52 & 43 & 47 \\
\hline & $\mathrm{S}$ & 36 & 31 & 38 & 39 & 34 & 34 & 47 & 56 & 52 & 43 & 34 & 39 \\
\hline
\end{tabular}

Table 6 shows the value of $\beta_{a^{t}}^{q f}$ for one exemplary time-period (from 8:00 am to 9:00 am). For the waiting links, the total loss caused by hazmat class 1 equals 60000, 80000 and 100000 for small, medium and large vehicle types, respectively. In the same way, for hazmat class 2 , the total loss of waiting links equals 30000,40000 , and 50000 for small, medium and large vehicle types, respectively.

Table 6. The value of $\beta_{a^{t}}^{q f}$ from 8 am to $9 \mathrm{am}$.

\begin{tabular}{|c|c|c|c|c|c|c|c|c|c|c|c|c|c|}
\hline \multirow{3}{*}{ Link } & \multicolumn{6}{|c|}{$\beta_{a^{t}}^{q f}$} & \multirow{3}{*}{ Link } & \multicolumn{6}{|c|}{$\beta_{a^{t}}^{q f}$} \\
\hline & \multicolumn{3}{|c|}{$q=1$} & \multicolumn{3}{|c|}{$q=2$} & & \multicolumn{3}{|c|}{$q=1$} & \multicolumn{3}{|c|}{$q=2$} \\
\hline & $f=1$ & $f=2$ & $f=3$ & $f=1$ & $f=2$ & $f=3$ & & $f=1$ & $f=2$ & $f=3$ & $f=1$ & $f=2$ & $f=3$ \\
\hline $1 \rightarrow 2$ & 773 & 1031 & 1288 & 139 & 185 & 231 & $12 \rightarrow 13$ & 994 & 1326 & 1657 & 179 & 238 & 298 \\
\hline $2 \rightarrow 3$ & 709 & 945 & 1182 & 127 & 170 & 212 & $13 \rightarrow 14$ & 994 & 1326 & 1657 & 179 & 238 & 298 \\
\hline $3 \rightarrow 4$ & 1009 & 1345 & 1681 & 181 & 242 & 302 & $11 \rightarrow 14$ & 787 & 1050 & 1312 & 141 & 189 & 236 \\
\hline $5 \rightarrow 4$ & 1009 & 1345 & 1681 & 181 & 242 & 302 & $14 \rightarrow 15$ & 787 & 1050 & 1312 & 141 & 189 & 236 \\
\hline $6 \rightarrow 5$ & 1009 & 1345 & 1681 & 181 & 242 & 302 & $15 \rightarrow 16$ & 787 & 1050 & 1312 & 141 & 189 & 236 \\
\hline $3 \rightarrow 6$ & 887 & 1183 & 1479 & 159 & 213 & 266 & $14 \rightarrow 17$ & 787 & 1050 & 1312 & 141 & 189 & 236 \\
\hline $4 \rightarrow 7$ & 1009 & 1345 & 1681 & 181 & 242 & 302 & $18 \rightarrow 15$ & 787 & 1050 & 1312 & 141 & 189 & 236 \\
\hline $6 \rightarrow 9$ & 527 & 703 & 879 & 95 & 126 & 158 & $16 \rightarrow 19$ & 695 & 927 & 1159 & 125 & 167 & 208 \\
\hline $7 \rightarrow 8$ & 994 & 1326 & 1657 & 179 & 238 & 298 & $17 \rightarrow 18$ & 517 & 689 & 861 & 93 & 124 & 155 \\
\hline $9 \rightarrow 8$ & 787 & 1050 & 1312 & 141 & 189 & 236 & $18 \rightarrow 19$ & 517 & 689 & 861 & 93 & 124 & 155 \\
\hline $7 \rightarrow 10$ & 1009 & 1345 & 1681 & 181 & 242 & 302 & $19 \rightarrow 21$ & 695 & 927 & 1159 & 125 & 167 & 208 \\
\hline $8 \rightarrow 11$ & 994 & 1326 & 1657 & 179 & 238 & 298 & $20 \rightarrow 21$ & 695 & 927 & 1159 & 125 & 167 & 208 \\
\hline $10 \rightarrow 11$ & 994 & 1326 & 1657 & 179 & 238 & 298 & $9 \rightarrow 20$ & 695 & 927 & 1159 & 125 & 167 & 208 \\
\hline $10 \rightarrow 12$ & 994 & 1326 & 1657 & 179 & 238 & 298 & $17 \rightarrow 22$ & 695 & 927 & 1159 & 125 & 167 & 208 \\
\hline $12 \rightarrow 11$ & 994 & 1326 & 1657 & 179 & 238 & 298 & & & & & & & \\
\hline
\end{tabular}

The service rate on waiting links for small, medium and large size vehicles and for both hazmat classes are 2, 3 and 4 minutes per vehicle, respectively. Table 7 shows the number of each vehicle type available for each dispatcher. The fleet size is assumed so that shipping the total hazmat flow for each OD pair cannot be done only by one type of 
vehicle. Therefore, a combination of all types needs to move total cargos from each origin.

Table 7. The number of vehicles available in the network.

\begin{tabular}{cccccccc}
\hline $\begin{array}{c}\text { Dispatcher } \\
\text { ID }\end{array}$ & $\begin{array}{c}\text { Origin } \\
\text { node }\end{array}$ & $\begin{array}{c}\text { Destination } \\
\text { node }\end{array}$ & $\begin{array}{c}\text { OD } \\
\text { (million } \\
\text { liter) }\end{array}$ & $\begin{array}{c}\text { Hazmat } \\
\text { class }\end{array}$ & $\begin{array}{c}\text { No. small } \\
\text { vehicles }\end{array}$ & $\begin{array}{c}\text { No. medium } \\
\text { vehicles }\end{array}$ & $\begin{array}{c}\text { No. large } \\
\text { vehicles }\end{array}$ \\
\hline 1 & 1 & 21 & 2 & 1 & 100 & 70 & 60 \\
2 & 3 & 22 & 3 & 1 & 130 & 90 & 80 \\
3 & 1 & 21 & 2.6 & 2 & 110 & 80 & 70 \\
4 & 3 & 22 & 3.4 & 2 & 150 & 100 & 90 \\
\hline
\end{tabular}

\section{RESULTS AND DISCUSSION}

We had already mathematically formulated the research problem that can be used to solve small and some mediumsize problems optimally. Then, we developed a metaheuristic technique to enable us to solve large-size problems to achieve near-optimal Pareto solutions quickly. There was another advantage with the existence of the mathematical formulation: it helped us in tuning the parameters of the meta-heuristic solution and the validation process. To test the model, we have used some real data to ensure our observations are reliable. Some of the parameters are based on a case study. For the additional parameters, we have generated the rest of the required data. In this section, we intend to obtain some useful and salient insights for practitioners through sensitivity analyses of the developed metaheuristic technique. Six different analyses are performed based on the data about the road network of Singapore.

\subsection{Tradeoff between the Objective Functions}

We solve the model for the case problem. The results yield 17 non-dominated solutions with a computational time of 176.8 seconds. Figure 8 represents the tradeoff between the objective functions. The horizontal and vertical axes of Figure 8 are normalized by dividing them by the minimum observed values.

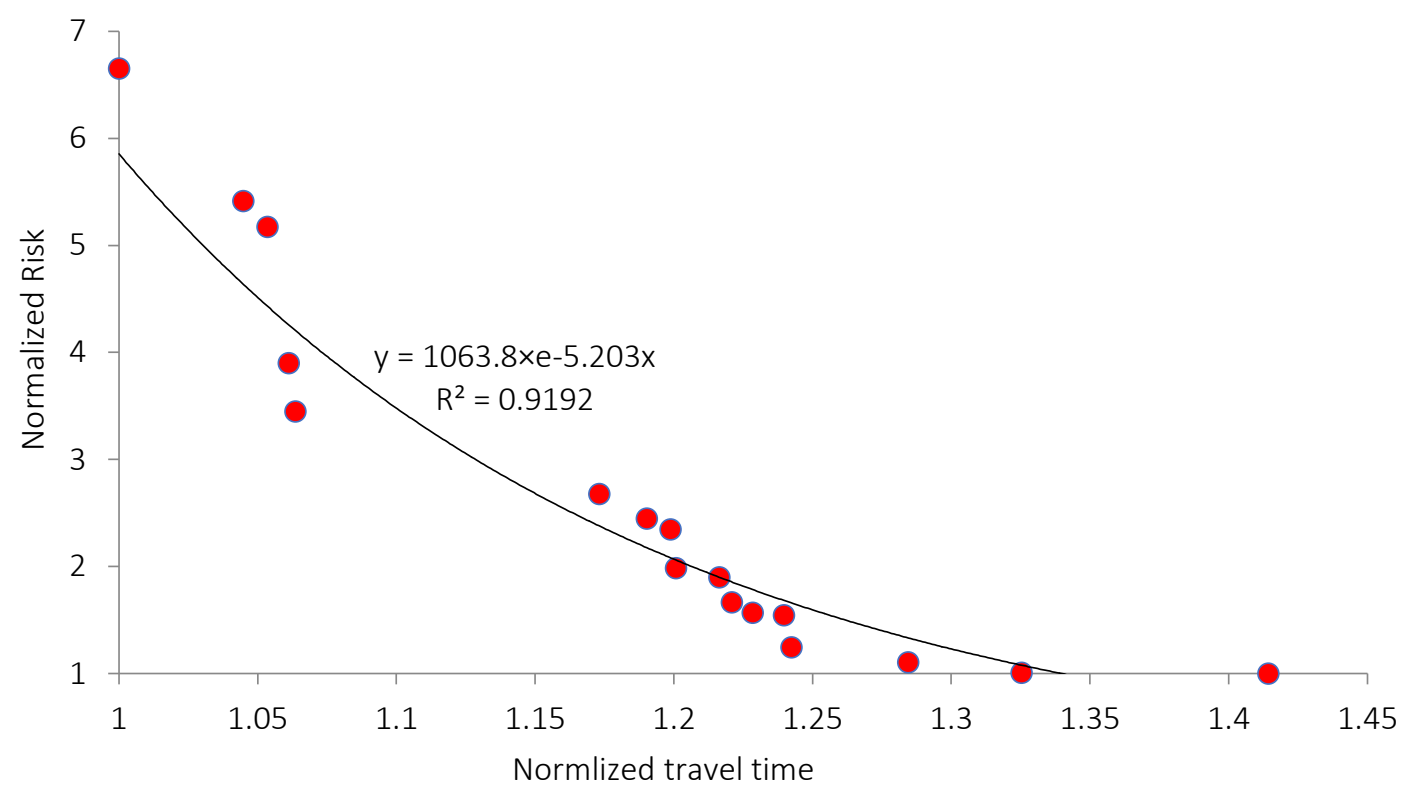

Figure 8. Tradeoff between the objective functions.

Observation 1: Figure 8 shows there is an exponential tradeoff between the two objectives. Decreasing travel time increases risk, exponentially. We approximated the tradeoff curve with several linear functions to show by what percentage the first objective function (risk) will decrease for a percent increment in the optimal value of the second objective function (travel time). This way, we can represent the tradeoff as a multi-segment function $\left(Z_{2}^{*}\right.$ is the optimal value of the second objective): 


$$
\frac{\partial Z_{1}}{\partial Z_{2}}=\left\{\begin{array}{lll}
-44.34\left(R^{2}=0.81\right) & \text { if } & 00.0 \% \leq \frac{\Delta z_{2}}{z_{2}^{*}} \leq 06.3 \% \\
-11.93\left(R^{2}=0.89\right) & \text { if } & 06.3 \% \leq \frac{\Delta z_{2}}{z_{2}^{*}} \leq 24.2 \% \\
-03.33\left(R^{2}=1.00\right) & \text { if } & 24.2 \% \leq \frac{\Delta z_{2}}{z_{2}^{*}} \leq 28.4 \% \\
-02.42\left(R^{2}=1.00\right) & \text { if } & 28.4 \% \leq \frac{\Delta z_{2}}{z_{2}^{*}} \leq 32.5 \% \\
-00.08\left(R^{2}=1.00\right) & \text { if } & 32.5 \% \leq \frac{\Delta z_{2}}{z_{2}^{*}} \leq 41.4 \%
\end{array}\right.
$$

Insight 1: The results show that by a little distancing (0 to 6\%) from the optimal solution for the travel time objective, the risk objective will improve significantly. If dispatchers move more than $24 \%$ of the optimal solution for the second objective, just a small improvement will occur in the first objective. Therefore, it is highly recommended that dispatchers pay attention to both risk and travel time objectives, even those only seek economic objectives (e.g. minimizing travel time). Moreover, we recommend that the risk-averse decision makers do not distance more than $24 \%$ of the optimal solution for the second objective because no significant improvement in the risk objective will occur. The literature on hazmat routing- scheduling shows that Zografos and Androutsopoulos (2004), Chang et al. (2005), Zografos and Androutsopoulos (2008), Androutsopoulos and Zografos (2010), Pradhananga et al. (2014a), Chai et al. (2017), Fang et al. (2017), and Men et al. (2020) investigated the tradeoff between the risk and cost objectives without further analysis on the risk and cost relationship. However, our tradeoff curve is similar to those presented by Androutsopoulos and Zografos (2010), Chai et al. (2017), Fang et al. (2017), and Men et al. (2020).

\subsection{Impact of the Number of Demons on the Objective Functions}

In this subsection, we analyze the impact of the number of demons on the tradeoff between the objective functions. The number of demons is increased from 1 to 5 gradually. Figure 9 shows the non-dominated solutions and the best fitting curve/ function for each case.

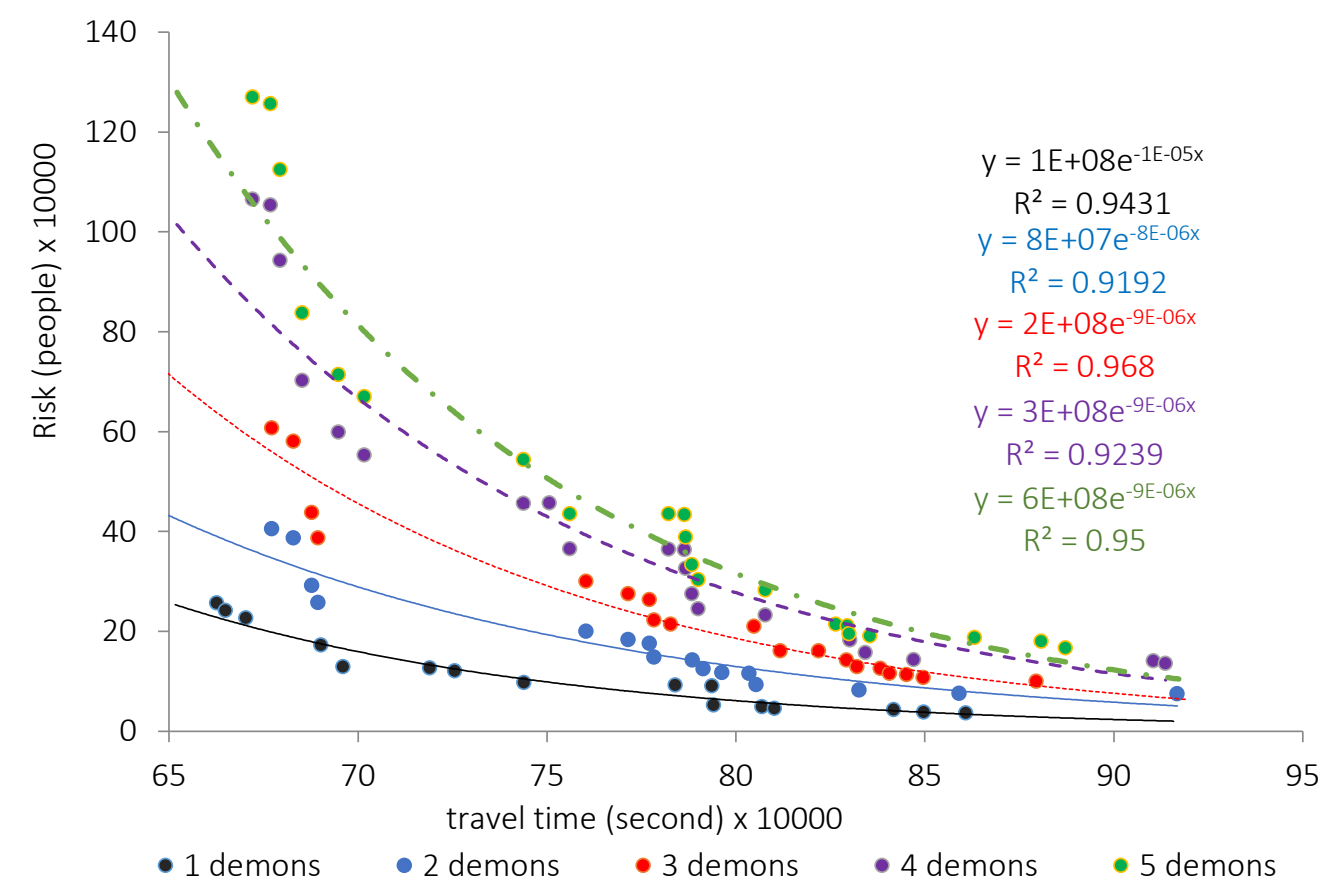

Figure 9. Non-dominated solutions for a different number of demons.

Observation 2: The tradeoff between the objective functions becomes sharper and more nonlinear by increasing the number of demons. For instance, increasing the number of demons from 3 to 4 and 5 results in increasing risk approximately 1.5 and 3 times, respectively. If we assign lower weights for the second objective function (i.e. travel time), the impact of increasing the number of demons on worsening the first objective function (i.e. risk) is more 
salient in comparison to higher weights for the second objective function (we can easily justify the rationality of this observation $^{2}$ ). In the case problem, when the travel time objective is 650000 seconds, one extra demon increases the first objective function by 240000 people, on average. However, when the travel time is 920000 seconds, one extra demon increases the first objective function by 16500 people, on average.

Insight 2: Risk-averse decision makers who prefer to increase travel time to reduce their risk (i.e. Pareto solutions on the right-hand side of the tradeoff diagram) should not have any concern about the number of accidents that may occur because by increasing the number of demons in this level, an insignificant increase will happen in the risk objective. On the other hand, risk-seeker decision makers (i.e. Pareto solutions on the left-hand side of the tradeoff diagram) do not care about the amount of risk (i.e. reflected by the number of demons). Therefore, we highly recommend that risk-neutral decision makers (i.e. Pareto solutions in the middle of the tradeoff diagram) estimate the number of demons accurately or even overestimate them to avoid an unexpected upsurge in the risk objective. Szeto et al. (2017) investigated the effect of changing the number of demons only on the risk objective function. Hence, they consider the decision maker (based on up to two demons) regardless of its risk attitudes.

\subsection{Impact of Including FIFO}

To investigate the impact of applying the FIFO discipline on the objectives, we performed sensitivity analyses on the service rate of waiting links and compared the results with those of excluding the FIFO discipline. The initial processing times (queueing discipline 1), as discussed in sections 7 are 2, 3 and 4 minutes for small, medium and large vehicle types, respectively. Then, we increased the processing times of vehicles two (queuing discipline 2), three (queuing discipline 3) and four (queuing discipline 4) times the initial values. Figure 10 shows the values of the objective functions for each queueing discipline as well as the case without the FIFO discipline.

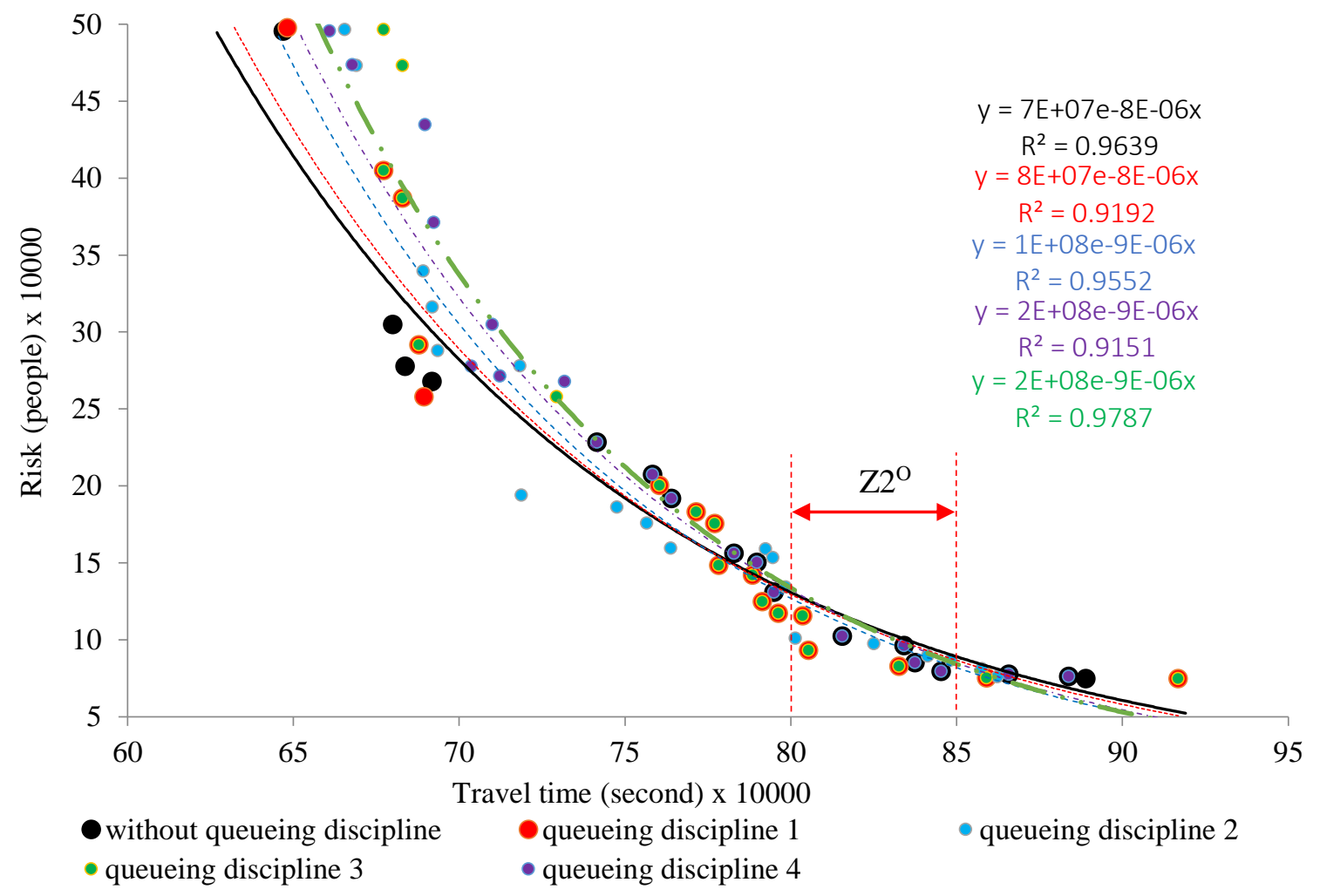

Figure 10. The effect of processing times on the tradeoff between the objective functions.

Observation 3: By increasing the processing times of waiting links, the tradeoff between the objective functions

${ }^{2}$ For example, we have $y_{1}=f_{1}(x)$ for one demon and $\mathrm{y}_{2}=f_{2}(x)$ for two demons (we can consider $y_{i}=a . e^{-b x}$ for $i$ demons). Therefore, $\Delta y \%=\left(y_{2}-y_{1}\right) / y_{1}=\left(y_{2} / y_{1}\right)-1$. Now, $y_{2} / y_{1}$ for $x<70$ is much bigger than $y_{2} / y_{1}$ for $x>80$. 
becomes sharper. There is a specific travel time value (between 80 and 85 in the case problem) shown by $Z 2^{\circ}$. For both $\mathrm{Z} 2>\mathrm{Z}^{\mathrm{O}}$ and $\mathrm{Z} 2<\mathrm{Z2}{ }^{\mathrm{O}}$, adding the FIFO discipline yields lower and higher values for Z1 (risk). Especially for condition $\mathrm{Z} 2<\mathrm{Z2}^{\mathrm{O}}$, the amount of discrepancy is higher than that of condition $\mathrm{Z} 2>\mathrm{Z2}^{\mathrm{O}}$.

Insight 3: We strongly recommend the managers, in particular those who weigh the travel time objective higher than the risk objective, to investigate the impact of FIFO in their hazmat routing-scheduling planning to estimate the values of risk and travel time objectives accurately.

\subsection{Impact of Having Safe Waiting Links}

In this subsection, we investigate the impact of converting the waiting links to some safe places for stopping hazmat cargos $^{3}$. Establishing safe places imposes an extra cost, but it would be recommended if it considerably improves the companies' objective functions (risk and travel time). Figure 11 shows the tradeoff between the two objective functions in two scenarios: scenario 1) this case is the initial model of section 4 with some loss for waiting links; scenario 2) in this case, the waiting links are eliminated by assuming that their total loss is equal to zero.

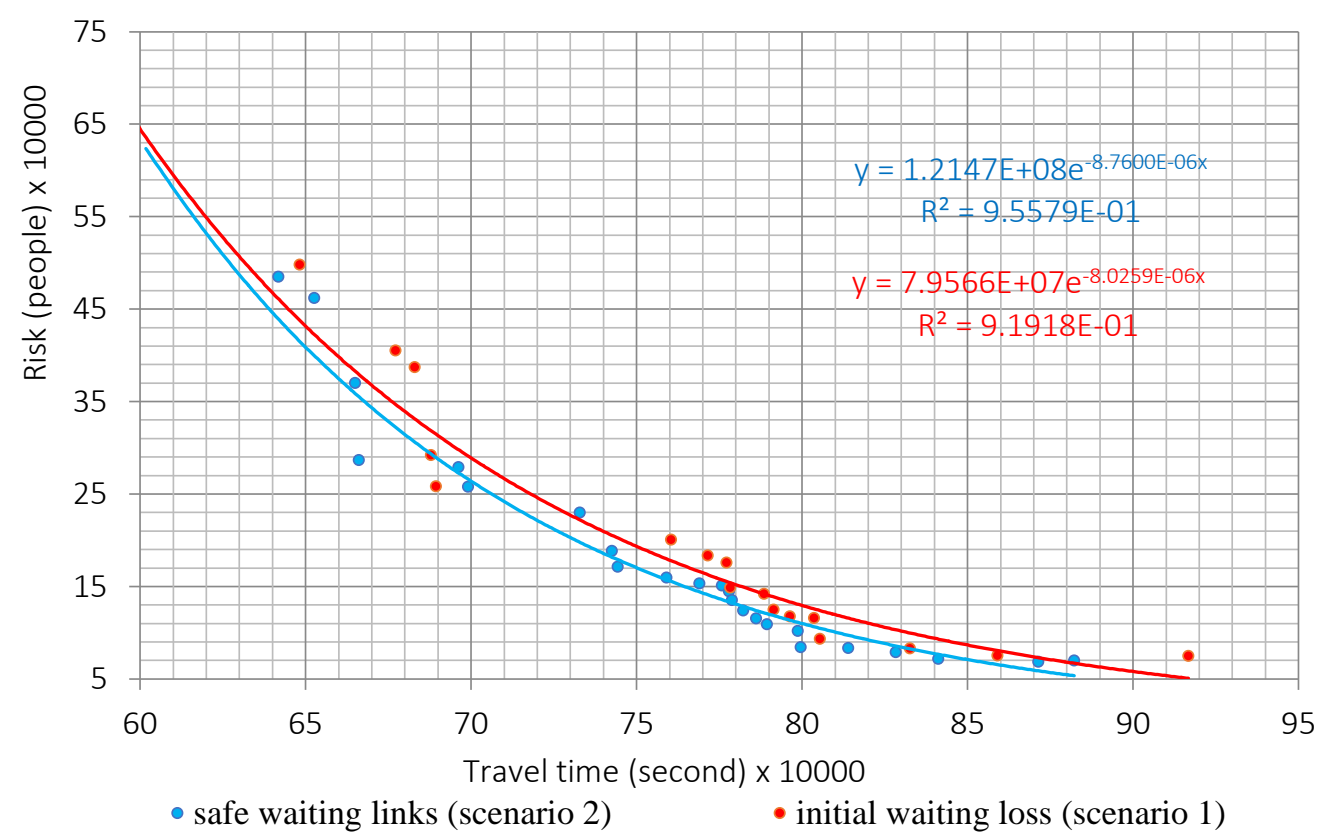

Figure 11. The impact of having safe waiting links.

Observation 4: Figure 11 shows that the tradeoff function of scenario 2 is always located below the tradeoff function of scenario 1 . As expected, the travel time and the risk of the network with safe waiting links is always less than the network with unsafe waiting links. The percentage of changing risk in all levels of travel time $\left(\forall x_{1}=x_{2}=\right.$ $x$ ) is computed by Equation (37).

$$
y^{\prime}=\frac{y_{1}-y_{2}}{y_{1}} 100=100-152.67 \times e^{-7.305 E-07 x} \quad \forall x_{1}=x_{2}=x
$$

In this equation, $y_{1}$ and $y_{2}$ are the risk of scenarios 1 and 2, respectively. $y^{\prime}$ is the reduced percentage of risk by converting the waiting links to safe places. For instance, for travel times equal to 650000,750000 , and 850000 , the reduced percentages of risk $\left(y^{\prime}\right)$ are approximately equal to 5,12 , and 18 percent.

Insight 4: From the results, we recommend fortifying waiting links to the risk-averse decision makers because they can receive a significantly reduced percentage of risk by converting the waiting links to safe places. Our findings support those presented by Ghaderi and Burdett (2019). They indicate that by increasing the disruption probability in transfer points, a slight increase in the values of risk and cost objectives occurs.

${ }^{3}$ Safe places are like safe parking areas in mega cities that can be used for hazmat vehicles during rush hours. 


\subsection{Impact of Mixed-Route Strategy}

In the algorithm, to reduce the risk in routing hazmat cargoes, one of the implemented strategies is increasing the number of routes used in the network. This strategy inhibits the concertation of hazmat cargos on a few links and disperses them on multiple links to reduce the risk in the network. However, the mixed-route strategy increases travel time. In this subsection, we investigate the impact of the number of routes used on the objective functions. We solve the model 20 times; each time, we increase the size of the route sets in Stage 1 from 1 to 200 with the step size of 10. The non-dominated solutions of five groups of routes (group 1: 1-40 routes; group 2: 41-80 routes; group 3: 81-120 routes; group 4: 121-160 routes; and group 5: 161-200 routes) are shown in Figure 12.

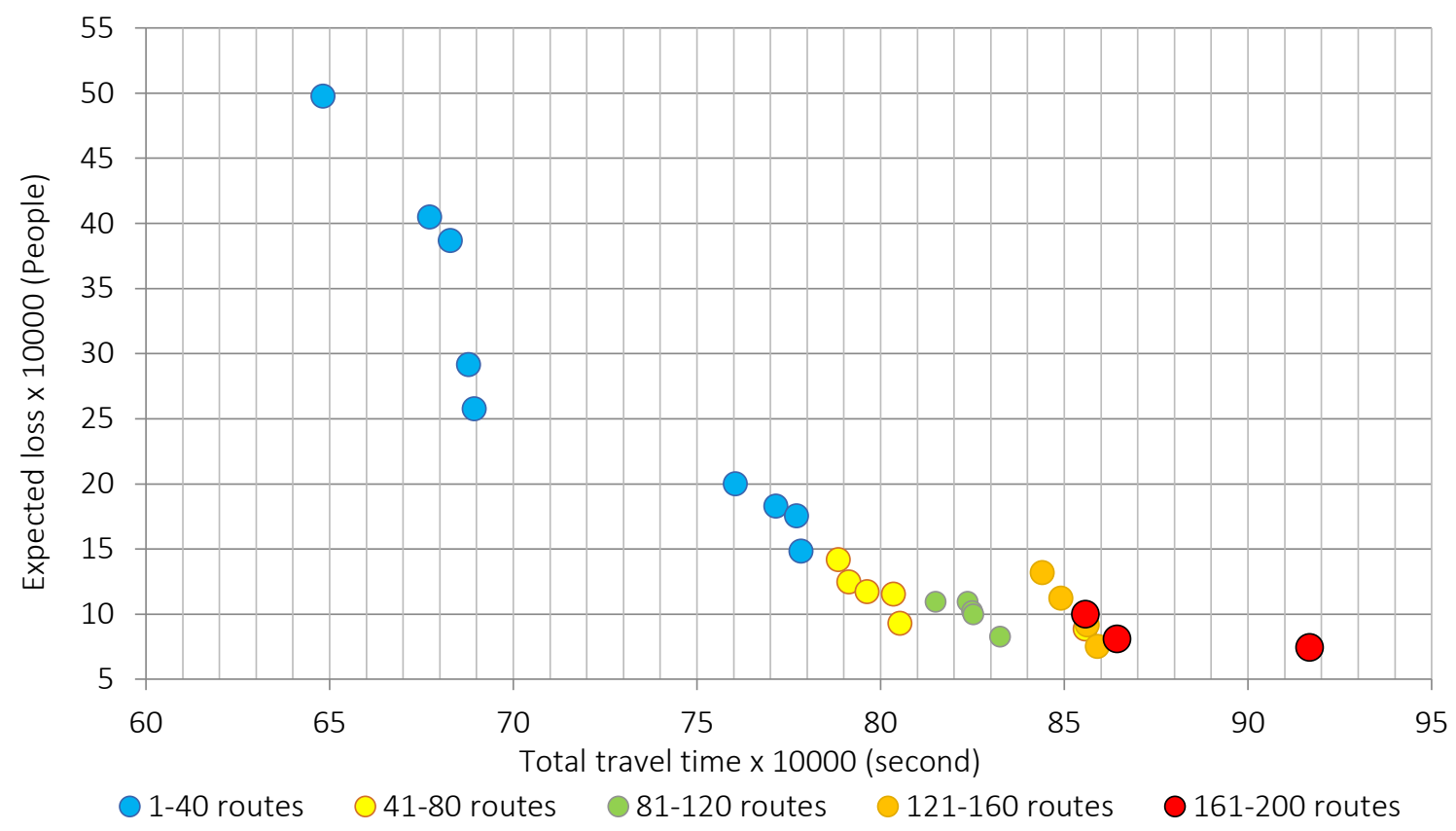

Figure 12. Impact of mixed-route strategy on the tradeoff of objective functions.

Observation 5: As expected, increasing the number of routes reduces the risk objective and increases the travel time objective. Comparing the average values of the objective functions of the categories yields the following objective: (1) When the size of the route set increases from Category 1 to Category 2, the risk decreases 60 percent but the travel time increases only 12 percent; (2) Increasing the route set's size to more than 80 routes does not significantly reduce the risk while it increases the travel time.

Insight 5: We recommend the route set's size between 41 to 80 routes (Category 2) to the conservative transportation companies of the Singapore network because it significantly reduces the risk Moreover, increasing the route set's size to more than 80 routes is not recommended at all.

\subsection{Impact of Fleet Size}

In the real world, hazmat shipping companies have a limited fleet size in some categories such as small, medium and large vehicle types. We identify the impact of different fleet sizes on the travel time and risk objectives. We assume all of the dispatchers have an unlimited fleet size (i.e. a large number of small, medium and large vehicle types are available). For each obtained non-dominated solution, the average size of vehicles used in routing hazmat cargos was calculated. For instance, if 10 vehicles with capacity 25,8 vehicles with capacity 20 and 4 vehicles with a capacity of 15 were utilized, the average vehicle size is equal to $\frac{10 \times 25+8 \times 20+4 \times 15}{10+8+4}=21.36$. Figure 13 shows the tradeoff between the objectives for the obtained non-dominated solutions and also the trend of changing the average vehicle size. 


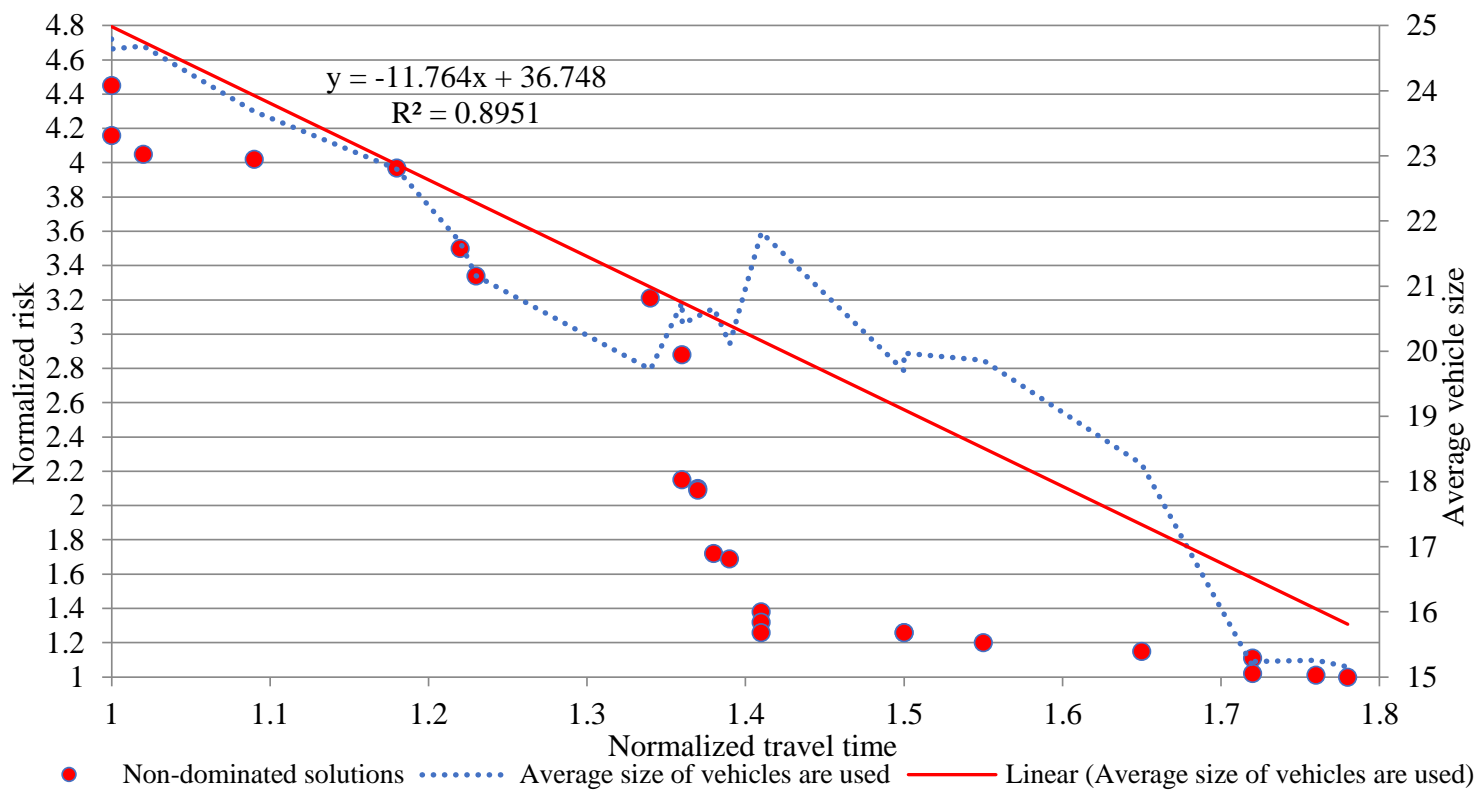

Figure 13. The best average sizes for different solutions.

Observation 6: The figure suggests the efficient average vehicle size for different levels of acceptable risk or travel time. Adjusting the average vehicle size in a range of 15 to 17 will help in decreasing the risk objective. Moreover, to minimize the travel time objective, the optimal average vehicle size is in a rage of 23 to 25 .

Insight 6: We highly recommend that risk-averse decision makers, who never accept a risk higher than $20 \%$ of the minimum risk, adjust the average vehicle size in a range of 15 to 17. Moreover, for the risk-seeking decision makers who prefer minimizing the travel time objective to minimizing the risk objective, setting the average vehicle size in a range of 23 to 25 is highly recommended. For other levels of acceptable risk or travel time, Figure 13 can suggest the efficient average vehicle size. Moving away from the suggested average vehicle size will not provide the expectations of risk and travel time objectives for such decision makers. Table 1 shows that 15 research papers consider multi-vehicle types. Additionally, we present analyses regarding the optimal fleet configuration for decision makers with various risk attitudes.

\subsection{A Summary of Insights}

We summarize the insights to explicitly clarify their practical meanings to logistics operations managers:

- We recommend the FIFO investigation in hazmat routing-scheduling, particularly to the dispatchers, weigh the travel time objective higher than the risk objective.

- Unlike risk-neutral decision makers, the risk-averse decision makers should not worry about the frequency of simultaneous incidents.

- The managers with any risk attitude should not merely focus on economic objective functions (i.e. travel time/ cost). Even a small distancing from the optimal travel time objective in favor of the risk objective can significantly reduce risk and the number of affected people.

- The risk-averse decision maker should keep the vehicle size in a range of 15 to 17 tons to balance between risk and travel time/cost objectives while observing fairness among dispatchers. In the same way, a risk-seeking decision maker should keep the vehicle size in a range of 23 to 25 tons. A risk-neutral planner should decide the vehicle sizes between two extremes.

- We observed that there is a particular number of established routes leading to the lowest risk objective. For the Singapore case, it is equal to 80 routes. Increasing the number of routes above this limit does not add any value.

- Fortifying the waiting links is recommended when dispatchers weigh the risk objective far higher than the travel 
time objective.

\section{CONCLUSION}

In this research, a game-theoretic, bi-objective hazmat routing-scheduling problem was investigated, in which the objective functions were minimization of risk and minimization of risk travel time. Considering the FIFO discipline for dispatchers, multiple hazmat classes, multiple vehicle types, and a limited fleet size were key aspects of the problem. A link-based game-theoretical approach with unknown incident probabilities was exploited to formulate the problem. This formulation helped us solve small and medium-size problems to obtain exact solutions. To solve large-size problems, a modified ALNS with SA was created. By using the proposed solution algorithm and real data from the Singapore road network, six different analyses were performed to provide lessons and insights for practitioners.

In this study, while the incident probability was unknown, the incident consequence was deterministic. This causes the demons to attack the links with high consequences. Hence, as a future direction, modeling the links' consequences as an uncertain parameter or with some categorized discrete values is recommended. Moreover, the research is based on the worst-case scenario that can incur high costs to firms. Defining measures other than the worst-case scenario can be investigated. 


\section{APPENDIX A}

The sets, indices, parameters and decision variables used in the paper are introduced as follow:

Sets

$G=[N, A] \quad:$ Original transportation network

$G^{\prime}=\left[N^{\prime}, A^{\prime}\right]:$ STEN of the original network

$N \quad$ : Set of nodes in the original network $G$

$S \quad:$ Set of scenarios representing the link selection strategies of the demons

$A \quad$ : Set of links in the original network $G$

$N_{o} \quad$ : Set of origin nodes in the original network $G$

$N_{d} \quad:$ Set of destination nodes in the original network $G$

$N_{i} \quad:$ Set of intermediate nodes in the original network $G$

$N^{\prime} \quad$ : Set of nodes in STEN

$A^{\prime} \quad$ : Set of links in STEN

$N^{t} \quad$ : Set of original nodes in sub-network $G^{t}$

$A^{t} \quad:$ Set of original links in sub-network $G^{t}$

$S^{\prime} \quad:$ Set of dummy origin nodes in STEN

$R^{\prime} \quad:$ Set of dummy destination nodes in STEN

$A_{d} \quad:$ Set of dummy links in STEN

$A_{w} \quad:$ Set of waiting links in STEN

$B \quad$ : Set of vehicle types

\section{Indices}

$k, m \quad$ : Demons

$t \quad$ : Time-periods

$s \quad$ : Link selection scenarios of demons

$i, x, y \quad:$ Nodes in STEN

$a^{t} \quad:$ Links of STEN $\left(a^{t}=(x, y) \in A^{\prime}\right)$

$l^{t}{ }_{m} \quad$ : A link of STEN such that demon $m$ causes an incident on it

$q \quad$ : Hazmat classes

$f, f^{\prime} \quad:$ Vehicle types

$r s_{q}, r^{\prime} s_{q^{\prime}}^{\prime} \quad$ : A dispatcher who ships hazmat class $q / q^{\prime}$ from origin node $r / r^{\prime}$ to destination node $s / s^{\prime}$

\section{Input parameters}

$T \quad$ : Number of time-periods inside the planning horizon

$D \quad$ : Length of each time-period

H : Number of dispatchers

M : Number of demons

$U \quad$ : A sufficiently large positive number

$L \quad$ : A sufficiently small positive number

$\phi \quad:$ A constant positive value

$b\left(a^{t}\right) \quad:$ The time-period number that $a^{t}$ belongs to

$Q^{\prime}\left(r s_{q}\right) \quad$ : Hazmat class of dispatcher $r s_{q}$

$D^{\prime}\left(r s_{q}\right) \quad$ : Dummy destination node for dispatcher $r s_{q}$

$O^{\prime}\left(r s_{q}\right) \quad:$ Dummy origin node for dispatcher $r s_{q}$

$O\left(a^{t}\right) \quad$ : Origin node of link $a^{t}$

$D\left(a^{t}\right) \quad$ : Destination node of link $a^{t}$

$C_{x} \quad:$ Maximum loss due to waiting at node $x$,

$\alpha_{a^{t}}^{q f}, \beta_{a^{t}}^{q f} \quad$ : Parameters of loss function associated with hazmat class $q$ and vehicle type $f$ over link $a^{t}$

$w_{f} \quad:$ Capacity of vehicles, type $f$ (ton/vehicle)

$d^{r s_{q}} \quad:$ Amount of hazmat cargos of dispatcher $r s_{q}$ (ton)

$F^{r s_{q f}} \quad:$ Number of vehicles of type $f$ that belong to dispatcher $r s_{q}$

$\lambda^{q^{\prime} f^{\prime}} \quad$ : Service time at queue of waiting links for hazmat class $q$ and vehicle type $f$

$t_{a^{t}}^{r s_{q f}} \quad:$ Travel time of link $a^{t}$ for dispatcher $r s_{q}$ with vehicles type $f$

$h \quad:$ A constant number between zero and one used in proposed metaheuristic solution approach

Temp : : Temperature used in proposed metaheuristic solution approach 


\begin{tabular}{|c|c|}
\hline$u_{s}$ & : Probability of scenario $s$ \\
\hline$p_{l_{m}^{t}}$ & : Probability that demon $m$ causes an incident on link $l^{t}$ \\
\hline$C_{a^{t}}(V)$ & : Loss of link $a^{t}$ in scenario $s$ \\
\hline$C_{a^{t}}(V)$ & : The maximum loss of link $a^{t}$ if an incident occurs on it \\
\hline$P$ & : Total expected payoff (risk that originates from demons' decisions) \\
\hline$V=\left[v_{l^{t}}^{q f}\right]$ & : Vector of hazmat flows \\
\hline$v_{a^{t}}^{r s}$ & : Flow of hazmat class $q$ using vehicle type $f$ on link $a^{t}$ that belongs to dispatcher $r s_{q}$ \\
\hline$v_{a^{t}}^{q f}$ & : Flow of hazmat class $q$ using vehicle type $f$ on link $a^{t}$ \\
\hline$\theta_{l^{t} m}$ & : Expected payoff of demon $m$ when it causes an incident on link $l^{t}$ \\
\hline$\pi^{m}$ & : Maximum expected payoff caused by demon $m$ \\
\hline$x_{a^{t},-m}$ & : Probability that link $a^{t}$ is selected by at least one demon other than demon $m$ \\
\hline$x_{a^{t}}$ & : Probability that link $a^{t}$ is selected by at least one demon \\
\hline$\eta_{a^{t}}^{r s_{q f}}$ & : Risk of dispatcher $r s_{q}$ who selects link $a^{t}$ and vehicle type $f$ \\
\hline$\sigma_{x}^{r s_{q f}}$ & $\begin{array}{l}\text { : Total accumulated travel time of dispatcher } r s_{q} \text { with vehicle type } f \text { departing from its origin node } \\
\text { up to the moment that the vehicle leaves node } x\end{array}$ \\
\hline$\sigma_{x}^{\prime}{ }^{r s_{q f}}$ & $\begin{array}{l}\text { : Share of the original and dummy links in the total accumulated travel time of dispatcher } r s_{q} \text { with } \\
\text { vehicle type } f \text { departing from its origin node up to the moment that the vehicle leaves node } x\end{array}$ \\
\hline$\sigma_{x}^{\prime \prime}{ }^{r s_{q f}}$ & $\begin{array}{l}\text { : Share of waiting links in the total accumulated travel time of dispatcher } r s_{q} \text { with vehicle type } f \\
\text { departing from its origin node up to the moment that the vehicle leaves node } x\end{array}$ \\
\hline$Z_{a^{t}}^{r s}$ & $\begin{array}{l}\text { : A binary variable that is equal to } 1 \text { if dispatcher } r s_{q} \text { with vehicle type } f \text { passes thought link } a^{t} \text {; and } \\
0 \text { otherwise. }\end{array}$ \\
\hline$Y_{i}^{r^{\prime} s^{\prime}} q^{\prime} f^{\prime}, r s_{q f}$ & $\begin{array}{l}\text { : A binary variable that is equal to } 1 \text { if dispatcher } r^{\prime} s^{\prime}{ }_{q} \text { with vehicle type } f^{\prime} \text { reaches node } i \text { sooner } \\
\text { than dispatcher } r s_{q} \text { with vehicle type } f \text {; and } 0 \text { otherwise. }\end{array}$ \\
\hline$W_{i}^{r s_{q f}, r^{\prime} s^{\prime}}{ }_{q^{\prime} f^{\prime}}$ & $\begin{array}{l}\text { : Delay time that is imposed on dispatcher } r s_{q} \text { with vehicle type } f \text { by dispatcher } r^{\prime} s^{\prime} q^{\prime} \text { with vehicle } \\
\text { type } f^{\prime} \text { on a waiting link whose origin is } i \text {. }\end{array}$ \\
\hline initial & : The initial solution produced in the proposed metaheuristic approach. \\
\hline . & : The new solution produced in each iteration of the proposed metaheuristic approach. \\
\hline & current solution available in each iteration of the proposed metaheuristic approach. \\
\hline
\end{tabular}

\section{APPENDIX B}

As mentioned, Constraints (13) through (16) compute the total accumulated travel time for dispatcher $r s_{q}$ using vehicle type $f$ from its origin node $(r)$ to node $x$. Node $x$ belongs to one of five different types of nodes in STEN: origin, destination, dummy origin, dummy destination and intermediate nodes. Constraints (13) to (16) hold for all node types. In Figure B1, we show the four categories (1, 2, 3 and 4) of nodes (note that category 1, unlike the others, encompasses two different types of nodes): (1) the origin and dummy origin nodes, (2) the intermediate nodes, (3) the destination nodes and (4) the dummy destination nodes. For each category, all possible arrival links are shown. $\sigma_{x}^{r s_{q f}}, \sigma_{x}^{\prime r s_{q f}}$, and $\sigma_{x}^{\prime \prime \prime}{ }_{x}^{r s_{q f}}$ for a node belonging to category (1) are equal to zero, according to constraint (13). For nodes belonging to category (2), the contributions of the crossed original and waiting links are computed separately by constraints (15) and (16). Finally, they are summed up by constraint (14). From the standpoint of dispatcher $r s_{q f}$, for nodes belonging to categories (3) and (4), the existence of an arriving waiting link is not possible. Therefore, constraints (14) and (15) are sufficient for calculation of $\sigma_{x}^{r s_{q f}}$. 


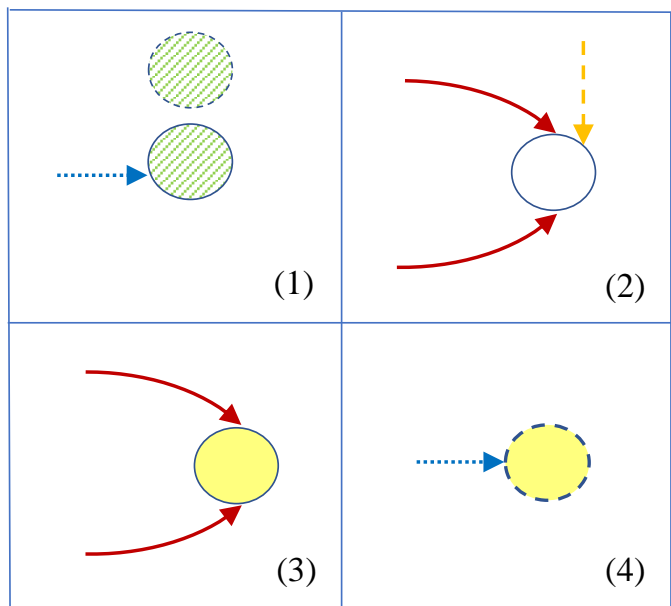

$\begin{aligned} & \longrightarrow \text { Original link } \\ & \ldots \ldots \text { Dummy link } \\ &--\triangleright \text { Waiting link } \\ &$\[ \text { Origin node } \]$\end{aligned}$

Dummy origin node

Destination node

Intermediate node

Dummy destination node

Figure B1. Calculating $\sigma_{x}^{r s} q f$ for different types of nodes in STEN.

APPENDIX C

Table $\mathrm{C} 1$ shows the possible scenarios and their descriptions.

Table C1: Possible scenarios for two vehicles arriving into waiting links to calculate their delays caused by FIFO.

\begin{tabular}{|c|c|}
\hline \# Scenario & Description of conditions \\
\hline 1 & 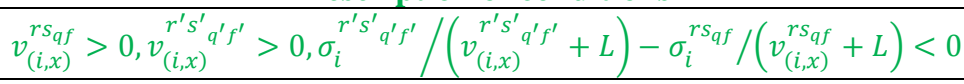 \\
\hline 2 & 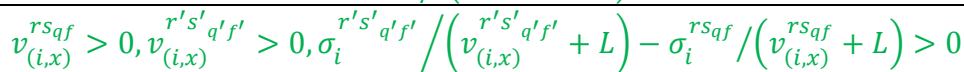 \\
\hline 3 & $v_{(i, x)}^{r s_{q f}}=0, v_{(i, x)}^{r^{\prime} s^{\prime} q^{\prime} f^{\prime}}>0, \sigma_{i}^{r^{\prime} s^{\prime}} q^{\prime} f^{\prime} /\left(v_{(i, x)}^{r^{\prime} s^{\prime} q^{\prime} f^{\prime}}+L\right)-\sigma_{i}^{r s_{q f}} /\left(v_{(i, x)}^{r s_{q f}}+L\right)<0$ \\
\hline 4 & $v_{(i, x)}^{r s_{q f}}=0, v_{(i, x)}^{r^{\prime} s^{\prime} q^{\prime} f^{\prime}}>0, \sigma_{i}^{r^{\prime} s^{\prime}} q^{\prime} f^{\prime} /\left(v_{(i, x)}^{r^{\prime} s^{\prime} q^{\prime} f^{\prime}}+L\right)-\sigma_{i}^{r s_{q f}} /\left(v_{(i, x)}^{r s_{q f}}+L\right)>0$ \\
\hline 5 & $v_{(i, x)}^{r s_{q f}}>0, v_{(i, x)}^{r^{\prime} s^{\prime} q^{\prime} f^{\prime}}=0, \sigma_{i}^{r^{\prime} s^{\prime} q^{\prime} f^{\prime}} /\left(v_{(i, x)}^{r^{\prime} s^{\prime} q^{\prime} f^{\prime}}+L\right)-\sigma_{i}^{r s_{q f}} /\left(v_{(i, x)}^{r s_{q f}}+L\right)<0$ \\
\hline 6 & $v_{(i, x)}^{r s_{q f}}>0, v_{(i, x)}^{r^{\prime} s^{\prime} q^{\prime} f^{\prime}}=0, \sigma_{i}^{r^{\prime} s^{\prime}} q^{\prime} f^{\prime} /\left(v_{(i, x)}^{r^{\prime} s^{\prime}} q^{\prime} f^{\prime}+L\right)-\sigma_{i}^{r s_{q f}} /\left(v_{(i, x)}^{r s_{q f}}+L\right)>0$ \\
\hline 7 & $v_{(i, x)}^{r s_{q f}}=0, v_{(i, x)}^{r^{\prime} s^{\prime} q^{\prime} f^{\prime}}=0, \sigma_{i}^{r^{\prime} s^{\prime}} q^{\prime} f^{\prime} /\left(v_{(i, x)}^{r^{\prime} s^{\prime}} q^{\prime} f^{\prime}+L\right)-\sigma_{i}^{r s_{q f}} /\left(v_{(i, x)}^{r s_{q f}}+L\right)<0$ \\
\hline 8 & 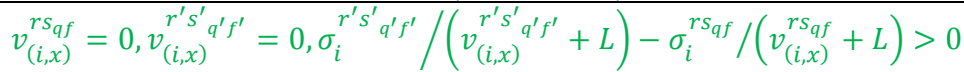 \\
\hline
\end{tabular}

Only in scenario 1 is a delay imposed on the vehicle type $f$ of dispatcher $r s_{q}$ by vehicle type $f^{\prime}$ of dispatcher

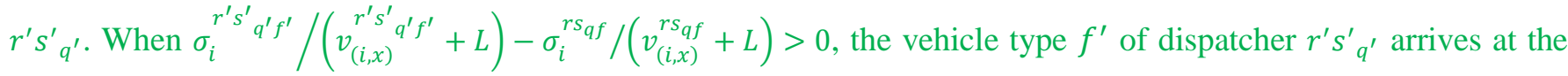
starting node of the waiting link later than the vehicle type $f$ of dispatcher $r s_{q}$. Therefore, no delay is added to the

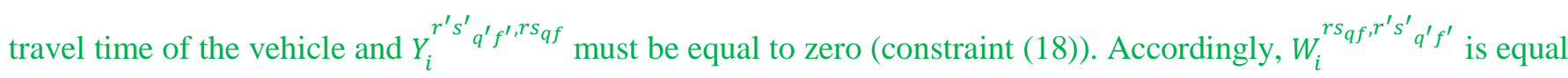
to zero based on constraint (19). Therefore, scenarios 2, 4, 6 and 8 never add any delay to the travel time of the

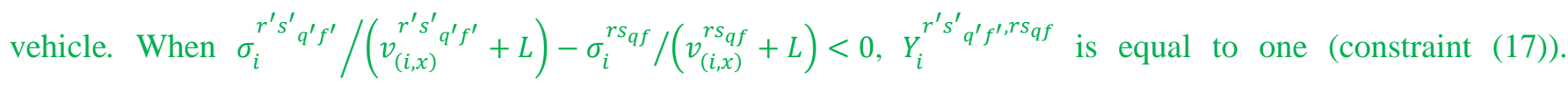
However, if $v_{(i, x)}^{r s_{q f}}$ or $v_{(i, x)}^{r^{\prime} s^{\prime} q^{\prime} f^{\prime}}$ is equal to zero, then $W_{i}^{r s_{q f}, r^{\prime} s^{\prime} q^{\prime} f^{\prime}}$ is equal to zero (constraint (19)). Therefore, scenarios 3,5 , and 7 never add any delay to the travel time of the vehicle either.

\section{APPENDIX D}

Sample problem A is comprised of 42 links and 22 nodes. Figure D1 shows the configuration of the related STEN. This network has two time-periods, two waiting links and two dispatchers. Nodes 1 and 9 are the origin and destination of the first dispatcher, respectively; the related demand is 150 tons of hazmat class 1 . Nodes 7 and 3 are the origin and destination of the second dispatcher, respectively; the related demand is 180 tons of hazmat class 2 . 
Two demons and three vehicle types are considered with a capacity of 10, 15, and 20 tons for each vehicle type, respectively. The amount of loss on the waiting links is considered to be zero. The length of each time-period is considered to be 10 units. The numbers of available vehicle types 1,2, and 3 for the first and second dispatcher are assumed to be 10,6 , and 8 and 12, 8, and 9, respectively. The amount of service time in the waiting links is $0.1,0.2$, and 0.3 units for the vehicle types, respectively. Service time is the amount of time taken to give a service to a vehicle in a queue and depends on the rate of service.

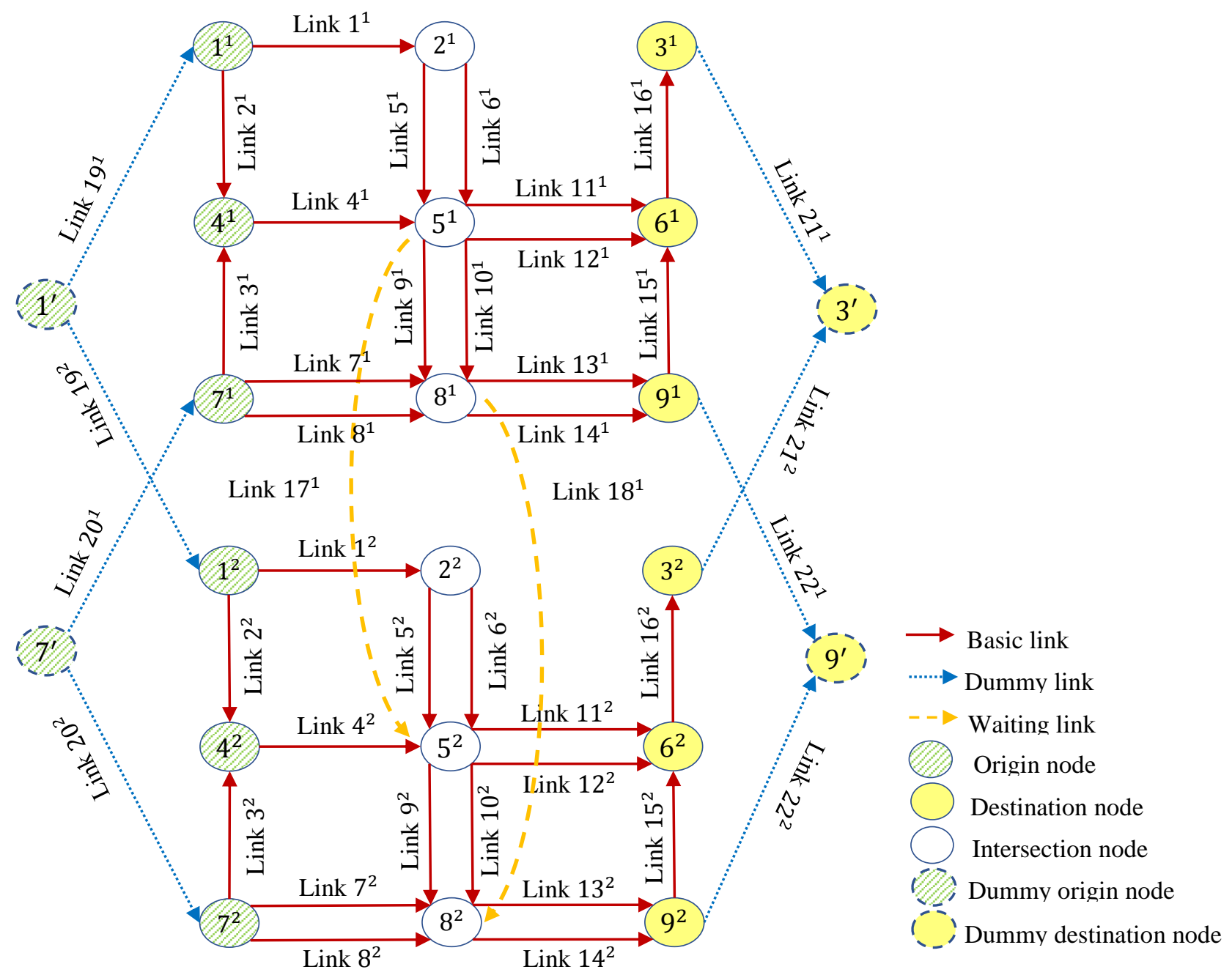

Figure D1. STEN for sample problem A.

We applied the AUGMECON method on this sample problem. The commercial solvers cannot solve the problem in a reasonable time. Therefore, the obtained Pareto-optimal solutions are reported only after one-hour running time; therefore, they are not guaranteed to be global optimum solutions. Table D1 is the payoff table for both objectives based on lexicographic optimization. Based on the results of the table, the range of the second objective is $32.6\left(r_{2}=\right.$ 32.6). What is more, five grid lines for finding Pareto-optimal solutions are assumed $\left(g_{2}=5\right)$. The results show that only for $i=1,2$, and 3 ( $i$ is the counter in AUGMECON in Figure E1 (Appendix E)) is a solution found within one hour. For $i>3$, no Pareto-optimal solution was obtained in one hour. Accordingly, Figure D2 shows the objective values for the five Pareto-optimal solutions related to this problem set. Tables D2, D3, and D4 show the $v_{a^{t}}^{r s_{q f}}$ for Pareto-optimal solutions 1, 2, and 3, respectively.

Table D1. The ideal and nadir solutions of two objectives for sample problem A.

\begin{tabular}{|c|c|c|}
\hline Solutions & Ideal solution & Nadir solution \\
\hline Objective function & 106.6 & 161.89 \\
\hline Fecond objective (Minimizing total travel time) & 83.3 & 115.9 \\
\hline
\end{tabular}




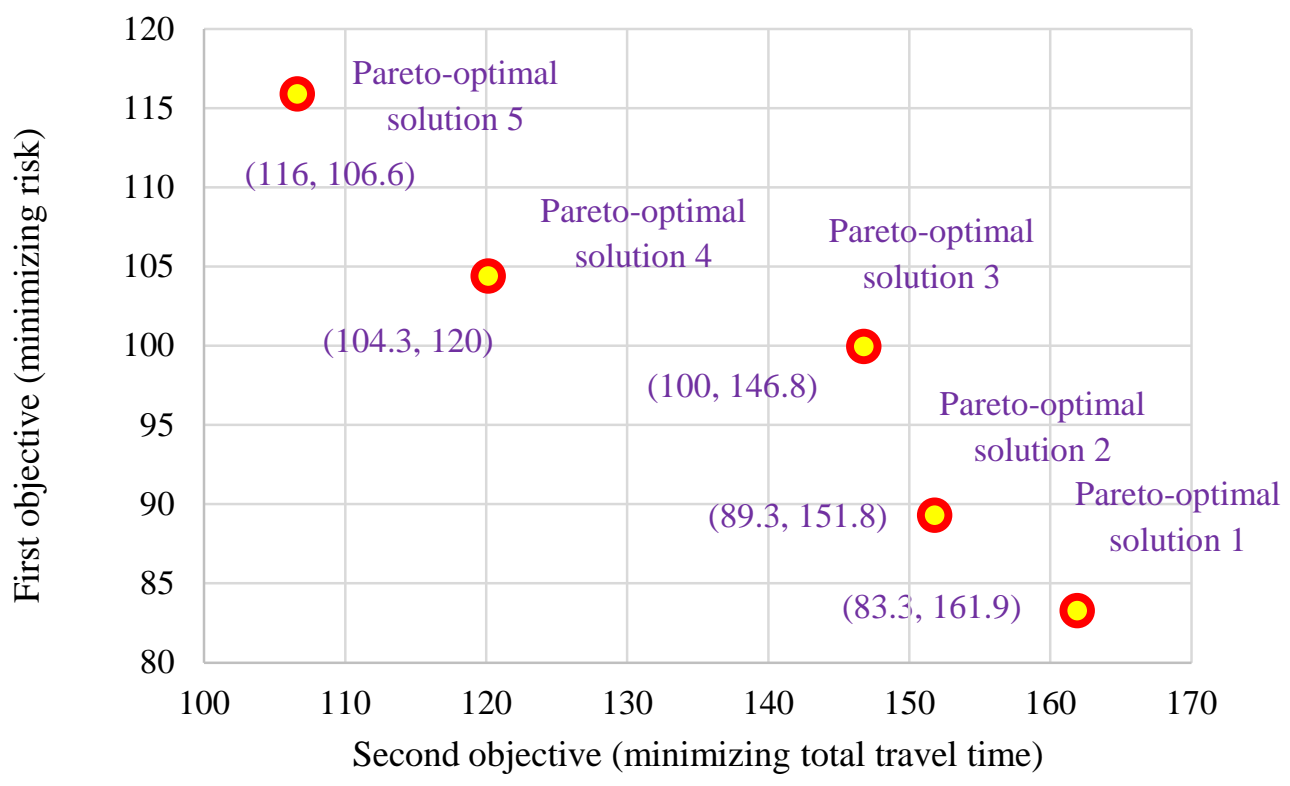

Figure D2. The Pareto-optimal solutions for sample problem A.

Table D2. The flows of hazmat vehicles on network links for Pareto-optimal solution 1.

\begin{tabular}{|c|c|c|c|c|c|c|c|c|c|c|c|c|c|}
\hline \multirow{3}{*}{ Link ID } & \multicolumn{6}{|c|}{$v_{a^{t}}^{r s_{q f}}$} & \multirow{3}{*}{ Link ID } & \multicolumn{6}{|c|}{$v_{a^{t}}^{r s_{q f}}$} \\
\hline & \multicolumn{3}{|c|}{$r s_{q}=1$} & \multicolumn{3}{|c|}{$r s_{q}=2$} & & \multicolumn{3}{|c|}{$r s_{q}=1$} & \multicolumn{3}{|c|}{$r s_{q}=2$} \\
\hline & $f=1$ & $f=2$ & $f=3$ & $f=1$ & $f=2$ & $f=3$ & & $f=1$ & $f=2$ & $f=3$ & $f=1$ & $f=2$ & $f=3$ \\
\hline $1^{1}$ & & & & & & & $11^{2}$ & & & & & 2 & \\
\hline $1^{2}$ & & & 6 & & & & $12^{1}$ & & & & & & 3 \\
\hline $2^{1}$ & & 1 & & & & & $12^{2}$ & & & & & & \\
\hline $2^{2}$ & & 1 & & & & & $13^{1}$ & & & & & & \\
\hline $3^{1}$ & & & & & & 3 & $13^{2}$ & & 1 & 6 & & 2 & \\
\hline $3^{2}$ & & & & & 2 & & $14^{1}$ & & 1 & & & & 3 \\
\hline $4^{1}$ & & 1 & & & & 3 & $14^{2}$ & & & & & & \\
\hline $4^{2}$ & & 1 & & & 2 & & $15^{1}$ & & & & & & 3 \\
\hline $5^{1}$ & & & & & & & $15^{2}$ & & & & & 2 & \\
\hline $5^{2}$ & & & 6 & & & & $16^{1}$ & & & & & & 6 \\
\hline $6^{1}$ & & & & & & & $16^{2}$ & & & & & 4 & \\
\hline $6^{2}$ & & & & & & & $17^{1}$ & & & & & & \\
\hline $7^{1}$ & & & & & & 3 & $18^{1}$ & & & & & & \\
\hline $7^{2}$ & & & & & & & $19^{1}$ & & 1 & & & & \\
\hline $8^{1}$ & & & & & & & $19^{2}$ & & 1 & 6 & & & \\
\hline $8^{2}$ & & & & & 2 & & $20^{1}$ & & & & & & 6 \\
\hline $9^{1}$ & & 1 & & & & & $20^{2}$ & & & & & 4 & \\
\hline $9^{2}$ & & 1 & 6 & & & & $21^{1}$ & & & & & & 6 \\
\hline $10^{1}$ & & & & & & & $21^{2}$ & & & & & 4 & \\
\hline $10^{2}$ & & & & & & & $22^{1}$ & & 1 & & & & \\
\hline $11^{1}$ & & & & & & & $22^{2}$ & & 1 & 6 & & & \\
\hline
\end{tabular}

Table D3. The flows of hazmat vehicles on network links for Pareto-optimal solution 3.

\begin{tabular}{|c|c|c|c|c|c|c|c|c|c|c|c|c|c|}
\hline \multirow{3}{*}{ Link ID } & \multicolumn{6}{|c|}{$v_{a^{t}}^{r s_{q f}}$} & \multirow{3}{*}{ Link ID } & \multicolumn{6}{|c|}{$v_{a^{t}}^{r s_{a f}}$} \\
\hline & \multicolumn{3}{|c|}{$r s_{q}=1$} & \multicolumn{3}{|c|}{$r s_{q}=2$} & & \multicolumn{3}{|c|}{$r s_{q}=1$} & \multicolumn{3}{|c|}{$r s_{q}=2$} \\
\hline & $f=1$ & $f=2$ & $f=3$ & $f=1$ & $f=2$ & $f=3$ & & $f=1$ & $f=2$ & $f=3$ & $f=1$ & $f=2$ & $f=3$ \\
\hline $1^{1}$ & & & & & & & $11^{2}$ & & & & & & \\
\hline $1^{2}$ & & & 5 & & & & $12^{1}$ & & & & & 2 & \\
\hline $2^{1}$ & & & 1 & & & & $12^{2}$ & & & & & & \\
\hline $2^{2}$ & & 2 & & & & & $13^{1}$ & & & & 1 & & \\
\hline $3^{1}$ & & & & & 2 & & $13^{2}$ & & 2 & 5 & & & 7 \\
\hline $3^{2}$ & & & & & & & $14^{1}$ & & & 1 & & & \\
\hline $4^{1}$ & & & 1 & & 2 & & $14^{2}$ & & & & & & \\
\hline $4^{2}$ & & 2 & & & & & $15^{1}$ & & & & 1 & & \\
\hline $5^{1}$ & & & & & & & $15^{2}$ & & & & & & 7 \\
\hline $5^{2}$ & & & 5 & & & & $16^{1}$ & & & & 1 & 2 & \\
\hline $6^{1}$ & & & & & & & $16^{2}$ & & & & & & 7 \\
\hline $6^{2}$ & & & & & & & $17^{1}$ & & & & & & \\
\hline $7^{1}$ & & & & 1 & & & $18^{1}$ & & & & & & \\
\hline $7^{2}$ & & & & & & 7 & $19^{1}$ & & & 1 & & & \\
\hline $8^{1}$ & & & & & & & $19^{2}$ & & 2 & 5 & & & \\
\hline $8^{2}$ & & & & & & & $20^{1}$ & & & & 1 & 2 & \\
\hline $9^{1}$ & & & & & & & $20^{2}$ & & & & & & 7 \\
\hline $9^{2}$ & & 2 & & & & & $21^{1}$ & & & & 1 & 2 & \\
\hline $10^{1}$ & & & 1 & & & & $21^{2}$ & & & & & & 7 \\
\hline $10^{2}$ & & & 5 & & & & $22^{1}$ & & & 1 & & & \\
\hline $11^{1}$ & & & & & & & $22^{2}$ & & 2 & 5 & & & \\
\hline
\end{tabular}


Table D4. The flows of hazmat vehicles on network links for Pareto-optimal solution 5.

\begin{tabular}{|c|c|c|c|c|c|c|c|c|c|c|c|c|c|}
\hline \multirow{3}{*}{ Link ID } & \multicolumn{6}{|c|}{$v_{a^{t}}^{r s_{q f}}$} & \multirow{3}{*}{ Link ID } & \multicolumn{6}{|c|}{$v_{a^{t}}^{r s_{q f}}$} \\
\hline & \multicolumn{3}{|c|}{$r s_{q}=1$} & \multicolumn{3}{|c|}{$r s_{q}=2$} & & \multicolumn{3}{|c|}{$r s_{q}=1$} & \multicolumn{3}{|c|}{$r s_{q}=2$} \\
\hline & $f=1$ & $f=2$ & $f=3$ & $f=1$ & $f=2$ & $f=3$ & & $f=1$ & $f=2$ & $f=3$ & $f=1$ & $f=2$ & $f=3$ \\
\hline $1^{1}$ & & & & & & & $11^{2}$ & & & & & & \\
\hline $1^{2}$ & & & 6 & & & & $12^{1}$ & & & & & & \\
\hline $2^{1}$ & & & & & & & $12^{2}$ & & & & & & \\
\hline $2^{2}$ & & 2 & & & & & $13^{1}$ & & & & & & \\
\hline $3^{1}$ & & & & & & 6 & $13^{2}$ & & 2 & 6 & & & 2 \\
\hline $3^{2}$ & & & & & & & $14^{1}$ & & & & 2 & & \\
\hline $4^{1}$ & & & & & & 6 & $14^{2}$ & & & & & & \\
\hline $4^{2}$ & & 2 & & & & & $15^{1}$ & & & & 2 & & \\
\hline $5^{1}$ & & & & & & & $15^{2}$ & & & & & & 2 \\
\hline $5^{2}$ & & & & & & & $16^{1}$ & & & & 2 & & 6 \\
\hline $6^{1}$ & & & & & & & $16^{2}$ & & & & & & 2 \\
\hline $6^{2}$ & & & 6 & & & & $17^{1}$ & & & & & & \\
\hline $7^{1}$ & & & & & & & $18^{1}$ & & & & & & \\
\hline $7^{2}$ & & & & & & & $19^{1}$ & & & & & & \\
\hline $8^{1}$ & & & & 2 & & & $19^{2}$ & & 2 & 6 & & & \\
\hline $8^{2}$ & & & & & & 2 & $20^{1}$ & & & & 2 & & 6 \\
\hline $9^{1}$ & & & & & & & $20^{2}$ & & & & & & 2 \\
\hline $9^{2}$ & & 2 & & & & & $21^{1}$ & & & & 2 & & 6 \\
\hline $10^{1}$ & & & & & & & $21^{2}$ & & & & & & 2 \\
\hline $10^{2}$ & & & 6 & & & & $22^{1}$ & & & & & & \\
\hline $11^{1}$ & & & & & & & $22^{2}$ & & 2 & 6 & & & \\
\hline
\end{tabular}

We fitted a linear trend line on the Pareto-optimal solutions shown in Figure D2. The results show, on average, one unit reduction in the travel time increases the risk 1.7 units. Moreover, comparison between the Pareto optimal solutions 1, 3 and 5 shows that choosing solution 3 over 1 leads to approximately $9 \%$ improvement in total travel time, but 20\% deterioration in risk. Shifting from solution 1 to 5 results in $34 \%$ improvement in travel time and $40 \%$ deterioration in risk. The number of established vehicles type 3 increases when shifting from solutions 1 to 3 and from 3 to 5 . This means whenever we give higher priority to the second objective (i.e. travel time), the dispatchers prefer to use larger vehicles (i.e. vehicles with higher capacity).

\section{APPENDIX E}

We introduce the AUGMECON method for a bi-objective optimization problem. The general form of the method was presented for maximization problems (Mavrotas, 2009) but we apply it for minimization problems (Figure E1).

$$
\begin{aligned}
& \operatorname{Min}\left(f_{1}(x)-\beta\left(s_{2} / r_{2}\right)\right) \\
& \text { s.t. } \\
& f_{2}(x)+s_{2}=e_{2} \\
& x \in S \text { and } s_{2} \in R^{+}
\end{aligned}
$$

Where:

$x:$ A vector of decision variables,

$S$ : The feasible region,

$f_{i}(x)$ : The $i$ th objective function,

$\beta$ : A small positive number (between $10^{-3}$ and $10^{-6}$ ),

$e_{2}$ : The maximum allowable level for $f_{2}(x)$

$s_{2}$ : A Positive shortage variable,

$r_{2}$ : The Range of $f_{2}(x)$

First, the method computes the payoff table from solving the corresponding lexicographic optimization. Practically, the lexicographic optimization for a bi-objective problem works as follows: i) optimize the primary objective function without considering the other objective function and obtain $f_{1}=z_{1}^{*}$; ii) optimize the second objective function by adding an extra constraint $f_{1}=z_{1}^{*}$ to find the Pareto solution for the problem. Second, in a repetitive loop changing the maximum allowable level for the second objective function $\left(e_{2}\right)$, the Pareto solutions are obtained. In each step, 
$e_{2}$ is changed automatically based on (E4).

$$
e_{2}=U B_{2}-\left(i_{2} \times r_{2}\right) / g_{2}
$$

Where $U B_{2}$ is the upper bound for $f_{2}$ (the maximum amount for it or the nadir solution), $g_{2}+1$ is the number of grid lines for dividing the range of $f_{2}$ that leads to $g_{2}$ equal intervals, and $i_{2}$ is the counter number for range intervals.

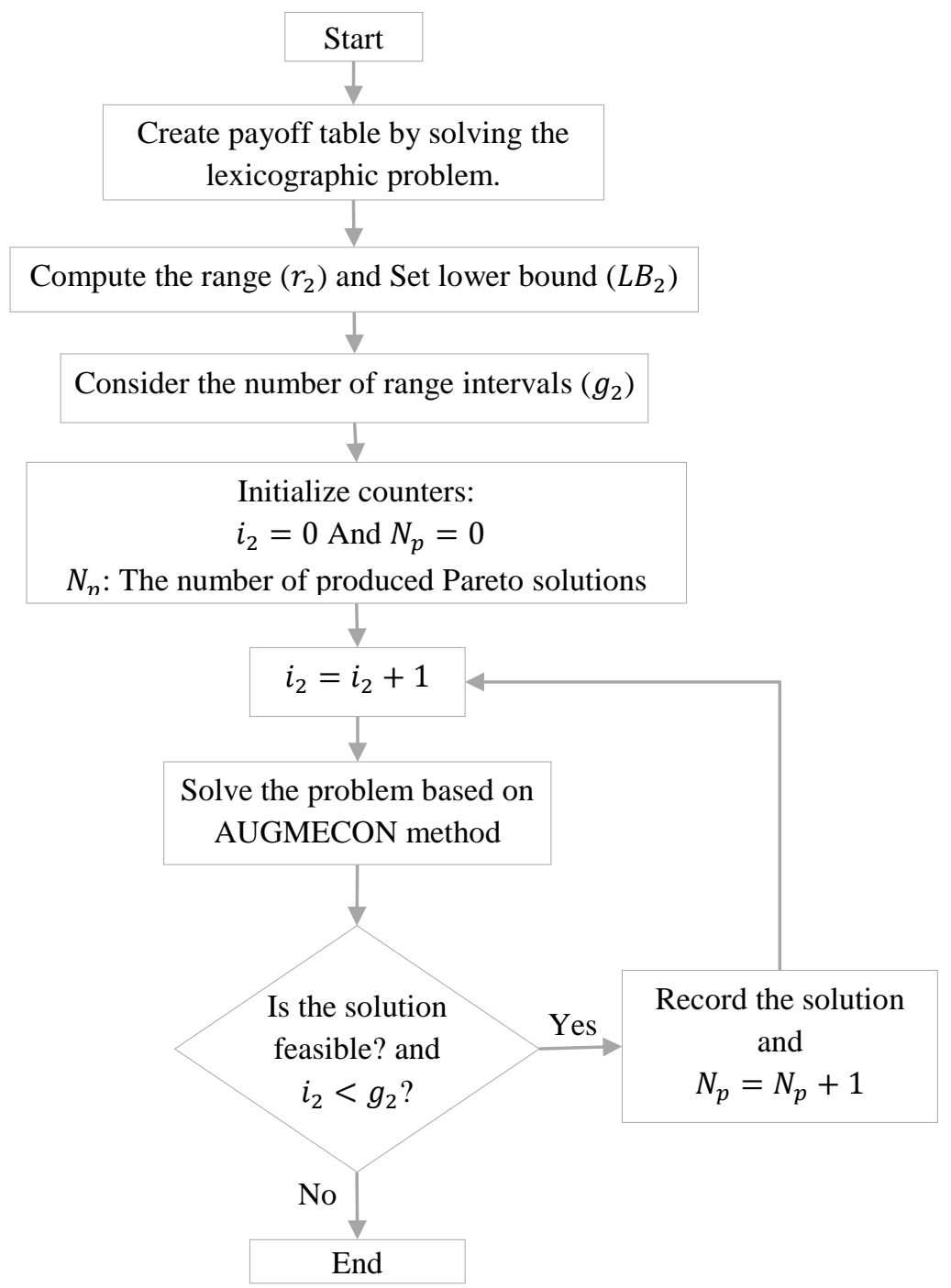

Figure E1. Flowchart of applying AUGMECON method on a bi-objective problem.

\section{REFERENCES}

Abuobidalla, O., Chen, M., \& Chauhan, S. (2019) A matheuristic method for planning railway freight transportation with hazard ous materials. Journal of Rail Transport Planning \& Management 10(1) 46-61.

Androutsopoulos, K. N., \& Zografos, K. G. (2010) Solving the bicriterion routing and scheduling problem for hazardous materials distribution. Transportation Research Part C: Emerging Technologies 18(5) 713-726.

Androutsopoulos, K. N., \& Zografos, K. G. (2012) A bi-objective time-dependent vehicle routing and scheduling problem for hazardous materials distribution. EURO Journal on Transportation and Logistics 1(1-2) 157-183.

Azi, N., Gendreau, M., \& Potvin, J.-Y. (2014) An adaptive large neighborhood search for a vehicle routing problem with multiple routes. Computers \& Operations Research 41(1) 167-173.

Bell, M. G. (2006) Mixed route strategies for the risk-averse shipment of hazardous materials. Networks and Spatial Economics 6(3-4) 253-265.

Bersani, C., Minciardi, R., Tomasoni, A. M., \& Sacile, R. (2010) Risk averse routing of hazardous materials with scheduled delays. In Security and Environmental Sustainability of Multimodal Transport (pp. 23-36) Springer, Dordrecht. Brander, A.

Bronfman, A., Marianov, V., Paredes-Belmar, G. and Lüer-Villagra, A. (2016) The maxisum and maximin-maxisum HAZMAT routing problems. Transportation Research Part E: Logistics and Transportation Review 93(1) 316-333.

Bula, G. A., Prodhon, C., Gonzalez, F. A., Afsar, H. M., \& Velasco, N. (2017) Variable neighborhood search to solve the vehicle routing problem for hazardous materials transportation. Journal of hazardous materials 324(Part B) 472-480.

Bureau of Transportation Statistics (2017a) Freight Facts \& Figures 2017 -Chapter 2: Freight Moved in Domestic and International Trade, https://www.bts.gov/bts-publications/freight-facts-and-figures/freight-facts-figures-2017-chapter-2-freight-moved, last visited on 17 August 2018.

Bureau of Transportation Statistics (2017b) Hazardous Materials Shipments by Transportation Mode, U.S. Department of Transportation, https://www.bts.gov/content/hazardous-materials-shipments-transportation-mode- 0 , last visited on 20 August 2018. 
Carotenuto, P., Giordani, S., \& Ricciardelli, S. (2007a) Finding minimum and equitable risk routes for hazmat shipments. Computers \& Operations Research 34(5) 1304-1327.

Carotenuto, P., Giordani, S., Ricciardelli, S., \& Rismondo, S. (2007b) A tabu search approach for scheduling hazmat shipments. Computers \& operations research 34(5) 1328-1350.

Cascetta, E., Russo, F., \& Vitetta, A. (1997) Stochastic user equilibrium assignment with explicit path enumeration: comparison of models and algorithms. IFAC Proceedings Volumes 30(8) 1031-1037.

Chai, H., He, R., Ma, C., Dai, C., \& Zhou, K. (2017) Path Planning and Vehicle Scheduling Optimization for Logistic Distribution of Hazardous Materials in Full Container Load. Discrete Dynamics in Nature and Society 2017(1) 1-13.

Chang, T.-S., Nozick, L. K., \& Turnquist, M. A. (2005) Multiobjective path finding in stochastic dynamic networks, with application to routing hazardous materials shipments. Transportation science 39(3) 383-399.

Chankong, V., \& Haimes, Y. Y. (2008) Multiobjective decision making: theory and methodology: Courier Dover Publications.

Chen, H.-K., \& Hsueh, C.-F. (1998) A model and an algorithm for the dynamic user-optimal route choice problem. Transportation Research Part B: Methodological 32(3) 219-234.

Cohon, J. L. (2004) Multiobjective programming and planning (Vol. 140): Courier Corporation.

Cohon, J. L., \& Marks, D. H. (1975) A review and evaluation of multiobjective programing techniques. Water Resources Research 11(2) 208-220.

DeLorenzo, J. P., Allen, J., Williams, D., \& Jensen, M. (2005) Safety, security, and efficiency benefits of technology in highway hazardous materials transportation applications. Paper presented at the Proceedings of the 2005 Mid-continent transportation research symposium, Ames, Iowa.

Demir, E., Bektaş, T., \& Laporte, G. (2012) An adaptive large neighborhood search heuristic for the pollution-routing problem. European Journal of Operational Research 223(2) 346-359.

Dijkstra, E. W. (1959) A note on two problems in connexion with graphs. Numerische Mathematik 1(1) $269-271$.

Drissi-Kaïtouni, O., \& Hameda-Benchekroun, A. (1992) A dynamic traffic assignment model and a solution algorithm. Transportation Science 26(2) 119-128.

Ehrgott, M. (2005) Multicriteria optimization (Vol. 491). Berlin: Springer Science \& Business Media.

Erkut, E., \& Alp, O. (2007) Integrated routing and scheduling of hazmat trucks with stops en route. Transportation Science 41(1) 107122.

Faghih-Roohi, S., Ong, Y.-S., Asian, S., \& Zhang, A. N. (2016) Dynamic conditional value-at-risk model for routing and scheduling of hazardous material transportation networks. Annals of Operations Research 247(2) 715-734.

Fan, T., Chiang, W.-C., \& Russell, R. (2015) Modeling urban hazmat transportation with road closure consideration. Transportation Research Part D: Transport and Environment 35(1) 104-115.

Fang, K., Ke, G. Y., \& Verma, M. (2017) A routing and scheduling approach to rail transportation of hazardous materials with demand due dates. European Journal of Operational Research 261(1) 154-168.

Ghaderi, A. and Burdett, R.L. (2019) An integrated location and routing approach for transporting hazardous materials in a bi-modal transportation network. Transportation research part E: logistics and transportation review 127(1) 49-65.

Gopalan, R., Kolluri, K.S., Batta, R. \& Karwan, M.H. (1990) Modeling equity of risk in the transportation of hazardous materials. Operations Research 38(6) 961-973.

Gordon, G. A., \& Young, R. R. (2015) Hazmat routing: Safety and security challenges. Paper presented at the 2015 Joint Rail Conference. San Jose, California, USA, March 23-26.

Grangier, P., Gendreau, M., Lehuédé, F., \& Rousseau, L.-M. (2016) An adaptive large neighborhood search for the two-echelon multiple-trip vehicle routing problem with satellite synchronization. European Journal of Operational Research 254(1) 80-91.

Greco, S., Figueira, J. and Ehrgott, M, (2016) Multiple criteria decision analysis. New York: Springer.

Haghani, A., \& Chen, Y.-J. (2003) Routing and scheduling for hazardous material shipments on networks with time dependent travel times. Paper presented at the TRB Annual Meeting CD-ROM.

Holeczek, N. (2019) Hazardous materials truck transportation problems: A classification and state of the art literature review. Transportation Research Part D: Transport and Environment 69(1) 305-328.

Hosseini, S. D., \& Verma, M. (2017) A Value-at-Risk (VAR) approach to routing rail hazmat shipments. Transportation Research Part D: Transport and Environment 54(1) 191-211.

Hosseini, S. D., \& Verma, M. (2018) Conditional value-at-risk (CVaR) methodology to optimal train configuration and routing of rail hazmat shipments. Transportation Research Part B: Methodological 110(1) 79-103.

Hosseinloo, S. H., Kanturska, U., Bell, M. G., \& Fonzone, A. (2012) Multi-path multi-criteria routing of hazardous materials in timedependent networks Transport of Dangerous Goods (pp. 151-165): Springer.

Hsu, Y. C., Walteros, J. L., \& Batta, R. (2018) Solving the petroleum replenishment and routing problem with variable demands and time windows. Annals of Operations Research 2018(1) 1-38.

Hu, H., Guo, S., Ma, H., Li, J., \& Li, X. (2017a) A Credibilistic Mixed Integer Programming Model for Time-Dependent Hazardous Materials Vehicle Routing Problem. Journal of Uncertain Systems 11(3) 163-175.

Huang, B., Cheu, R. L., \& Liew, Y. S. (2004) GIS and genetic algorithms for HAZMAT route planning with security considerations. International Journal of Geographical Information Science 18(8) 769-787.

Hwang, C.-L., \& Masud, A. S. M. (2012) Multiple objective decision making-methods and applications: a state-of-the-art survey (Vol. 164): Springer Science \& Business Media.

Jiang, P., Men, J., Xu, H., Zheng, S., Kong, Y., \& Zhang, L. (2020) A Variable Neighborhood Search-Based Hybrid Multiobjective Evolutionary Algorithm for HazMat Heterogeneous Vehicle Routing Problem With Time Windows. IEEE Systems Journal 1-12.

Kang, Y., Batta, R., \& Kwon, C. (2014) Generalized route planning model for hazardous material transportation with var and equity considerations. Computers \& Operations Research 43(1) 237-247.

Karbassi Yazdi, A., Kaviani, M. A., Emrouznejad, A., \& Sahebi, H. (2019) A binary particle swarm optimization algorithm for ship routing and scheduling of liquefied natural gas transportation. Transportation Letters 1-10.

Kazantzi, V., Kazantzis, N., \& Gerogiannis, V. C. (2011) Risk informed optimization of a hazardous material multi-periodic transportation model. Journal of Loss Prevention in the Process Industries 24(6) 767-773.

Kovacs, A. A., Parragh, S. N., Doerner, K. F., \& Hartl, R. F. (2012) Adaptive large neighborhood search for service technician routing and scheduling problems. Journal of scheduling 15(5) 579-600.

Larson, R. C. (1987). OR forum-perspectives on queues: Social justice and the psychology of queueing. Operations research 35(6) 
895-905.

Li, R., \& Leung, Y. (2011) Multi-objective route planning for dangerous goods using compromise programming. Journal of Geographical Systems 13(3) 249-271.

Li, R., Leung, Y., Huang, B., \& Lin, H. (2013) A genetic algorithm for multiobjective dangerous goods route planning. International Journal of Geographical Information Science 27(6) 1073-1089.

Liu, L.-p., Wang, M.-Z., \& Li, S.-x. (2019) Route Optimization Model for Hazardous Materials Transportation Considering Traffic Restrictions and Delivery Time Windows. DEStech Transactions on Computer Science and Engineering 362-368.

Mahmoudabadi, A., \& Seyedhosseini, S. M. (2014) Solving Hazmat Routing Problem in chaotic damage severity network under emergency environment. Transport policy 36(1) 34-45.

Mavrotas, G. (2007) Generation of efficient solutions in multiobjective mathematical programming problems using GAMS. Effective implementation of the e-constraint method. Lecturer, Laboratory of Industrial and Energy Economics, School of Chemical Engineering. National Technical University of Athens.

Mavrotas, G. (2009) Effective implementation of the $\varepsilon$-constraint method in multi-objective mathematical programming problems. Applied mathematics and computation 213(2) 455-465.

Men, J., Jiang, P., Xu, H., Zheng, S., Kong, Y., Hou, P., \& Wu, F. (2020) Robust multi-objective vehicle routing problem with time windows for hazardous materials transportation. IET Intelligent Transport Systems 14(3) 154-163.

Meng, Q., Lee, D.-H., \& Cheu, R. L. (2005) Multiobjective vehicle routing and scheduling problem with time window constraints in hazardous material transportation. Journal of Transportation Engineering 131(9) 699-707.

Mishra, M., Han, X., Ayala, D. F., Sidoti, D., Pattipati, K., An, W., \& Klienman, D. L. (2014) Multi-objective asset routing problem within a dynamic environment. Paper presented at the Advance Computing Conference (IACC), 2014 IEEE International.

Mohri, S.S., Karimi, H., Kordani, A.A. \& Nasrollahi, M. (2018) Airline hub-and-spoke network design based on airport capacity envelope curve: a practical view. Computers \& Industrial Engineering 125(1) 375-393.

Nozick, L. K., List, G. F., \& Turnquist, M. A. (1997) Integrated routing and scheduling in hazardous materials transportation. Transportation Science 31(3) 200-215.

Özceylan, E., Erbaş, M., Çetinkaya, C., \& Kabak, M. (2017) A GIS-based risk reduction approach for the hazardous materials routing problem in Gaziantep. Human and Ecological Risk Assessment: An International Journal, 23(6), 1437-1453.

Pamučar, D., Ljubojević, S., Kostadinović, D., \& Đorović, B. (2016) Cost and risk aggregation in multi-objective route planning for hazardous materials transportation-A neuro-fuzzy and artificial bee colony approach. Expert Systems with Applications 65(1) 115 .

PHMSA (Pipeline and Hazardous Materials Safety Administration) (2018), Incident Statistics, U.S. Department of Transportation, Yearly Incident Summary Reports, https://www.phmsa.dot.gov/hazmat-program-management-data-and-statistics/dataoperations/incident-statistics, last visited on 17 August 2018.

Pisinger, D., \& Ropke, S. (2010) Large neighborhood search, Handbook of Metaheuristics (pp. 399-419): Springer.

Pradhananga, R., Hanaoka, S., \& Sattayaprasert, W. (2011) Optimisation model for hazardous material transport routing in Thailand. International Journal of Logistics Systems and Management 9(1) 22-42.

Pradhananga, R., Taniguchi, E., \& Yamada, T. (2009) Optimization of vehicle routing and scheduling problem with time window constraints in hazardous material transportation. Paper presented at the Proceedings of the Eastern Asia Society for Transportation Studies Vol. 7 (The 8th International Conference of Eastern Asia Society for Transportation Studies, 2009)

Pradhananga, R., Taniguchi, E., \& Yamada, T. (2010) Ant colony system based routing and scheduling for hazardous material transportation. Procedia-Social and Behavioral Sciences 2(3) 6097-6108.

Pradhananga, R., Taniguchi, E., Yamada, T., \& Qureshi, A. G. (2014a) Bi-objective decision support system for routing and scheduling of hazardous materials. Socio-Economic Planning Sciences 48(2) 135-148.

Pradhananga, R., Taniguchi, E., Yamada, T., \& Qureshi, A. G. (2014b) Environmental analysis of Pareto optimal routes in hazardous material transportation. Procedia-Social and Behavioral Sciences 125(1) 506-517.

Pradhananga, R., Taniguchi, E., Yamada, T., \& Qureshi, A. G. (2016) Risk of traffic incident delay in routing and scheduling of hazardous materials. International Journal of Intelligent Transportation Systems Research 14(1) 50-63.

Rahman, A., Fiondella, L., \& Lownes, N. E. (2014) A bi-objective approach to evaluate highway routing and regulatory strategies for hazardous materials transportation. Paper presented at the Journal of the Transportation Research Forum.

Ran, B., \& Boyce, D. E. (1996) A link-based variational inequality formulation of ideal dynamic user-optimal route choice problem. Transportation Research Part C: Emerging Technologies 4(1) 1-12.

Ran, B., Hall, R. W., \& Boyce, D. E. (1996) A link-based variational inequality model for dynamic departure time/route choice. Transportation Research Part B: Methodological 30(1) 31-46.

Ribeiro, G. M., \& Laporte, G. (2012) An adaptive large neighborhood search heuristic for the cumulative capacitated vehicle routing problem. Computers \& operations research 39(3) 728-735.

Roman, C., \& Rosehart, W. (2006) Evenly distributed Pareto points in multi-objective optimal power flow. IEEE Transactions on Power Systems 21(2) 1011-1012.

Romero, N., Nozick, L.K. and Xu, N. (2016) Hazmat facility location and routing analysis with explicit consideration of equity using the Gini coefficient. Transportation research part E: logistics and transportation review 89(1) 165-181.

Ropke, S., \& Pisinger, D. (2006) An adaptive large neighborhood search heuristic for the pickup and delivery problem with time windows. Transportation Science 40(4) 455-472.

Sahnoon, I., Shanableh, A., \& Hamad, K. (2016) Risk-based GIS model for hazardous materials routing in Sharjah, United Arab Emirates as a case study. International Journal of Sustainable Society 8(2) 96-108.

Schmitt, B. H., Dube, L., \& Leclerc, F. (1992). Intrusions into waiting lines: does the queue constitute a social system? Journal of Personality and Social Psychology 63(5) 806-815.

Seaco (2018). T20 \& T22 Specialised tanks, from https://www.seacoglobal.com/equipment/tank-containers/t20-t22-specialisedtanks/t20-t22-specialised-tanks/ (last visited on 17 August 2018)

Shen, X., Xie, C., Liu, H., \& Qiu, Z. (2013) Model and algorithm for routing and scheduling problem in hazardous materials transportation network. JNW 8(5) 1027-1034.

Sun, L., Karwan, M. H., \& Kwon, C. (2016) Implications of cost equity consideration in hazmat network design. Transportation Research Record: Journal of the Transportation Research Board 2567(1) 67-77.

Sun, X., Feng, S., \& Li, Z. (2018) An iterative optimization model for hazardous materials transport with demand changes. KSCE 
Journal of Civil Engineering 22(1) 292-297.

Szeto, W. (2013) Routing and scheduling hazardous material shipments: Nash game approach. Transportmetrica B: transport dynamics 1(3) 237-260.

Szeto, W. Y., Farahani, R. Z., \& Sumalee, A. (2017) Link-based multi-class hazmat routing-scheduling problem: A multiple demon approach. European Journal of Operational Research 261(1) 337-354.

Timajchi, A., Al-e-Hashem, S. M. M., \& Rekik, Y. (2019) Inventory routing problem for hazardous and deteriorating items in the presence of accident risk with transshipment option. International Journal of Production Economics 209(1) 302-315.

Toumazis, I., \& Kwon, C. (2013) Routing hazardous materials on time-dependent networks using conditional value-at-risk. Transportation Research Part C: Emerging Technologies 37(1) 73-92.

U.S. Department of Energy (2019) Energy Information Administration, Movements between PAD Districts, https://www.eia.gov/petroleum/data.php, last visited on 28 March 2020.

Van der Zijpp, N., \& Catalano, S. F. (2005) Path enumeration by finding the constrained K-shortest paths. Transportation Research Part B: Methodological 39(6) 545-563.

Verma, M. (2009) A cost and expected consequence approach to planning and managing railroad transportation of hazardous materials. Transportation Research Part D: Transport and Environment 14(5) 300-308.

Wang, N., Zhang, M., Che, A., \& Jiang, B. (2017) Bi-objective vehicle routing for hazardous materials transportation with no vehicles travelling in echelon. IEEE Transactions on Intelligent Transportation Systems.

Xiang, Q., Li, H., Huang, B., \& Li, R. (2012) Improved ant colony optimization for multi-objective route planning of dangerous goods. Paper presented at the Natural Computation (ICNC), 2012 Eighth International Conference on.

Yates, J., Batta, R., \& Karwan, M. (2011) Optimal placement of sensors and interception resource assessment for the protection of regional infrastructure from covert attack. Journal of Transportation Security 4(2) 145-169.

Yates, J., Batta, R., Karwan, M., \& Casas, I. (2012) Establishing public policy to protect critical infrastructure: Finding a balance between exposure and cost in Los Angeles County. Transport Policy 24(1) 109-117.

Yates, J., \& Casas, I. (2012) Role of spatial data in the protection of critical infrastructure and homeland defense. Applied Spatial Analysis and Policy 5(1) 1-23.

Yates, J. T. (2008) Network-based risk mitigation and resource evaluation in the transportation of hazardous materials and terrorist threat: State University of New York at Buffalo.

Yuan, W., Wang, J., Li, J., Yan, B., \& Wu, J. (2017) Two-stage heuristic algorithm for a new model of hazardous material multi-depot vehicle routing problem. Paper presented at the UK Workshop on Computational Intelligence.

Zhou, R., \& Soman, D. (2008) Consumers' waiting in queues: The role of first- order and second- order justice. Psychology \& Marketing 25(3) 262-279.

Zografos, K. G., \& Androutsopoulos, K.N. (2004) A heuristic algorithm for solving hazardous materials distribution problems. European Journal of Operational Research 152(2) 507-519.

Zografos, K.G. and Androutsopoulos, K.N. (2008) A decision support system for integrated hazardous materials routing and emergency response decisions. Transportation Research Part C: Emerging Technologies 16(6) 684-703. 


\section{Kingston University London \\ Faculty of Business \& Law}

4 June 2020

Prof. Dr. Jason Choi (Editor-in-Chief)

Transportation Research Part E: Logistics and Transportation Review

Manuscript reference number: TRE_2020_230.R3

Dear Prof. Dr. Jason Choi (Editor-in-Chief)

I hope you are fine.

Attached is the third revised version of the paper "Fairness in Hazmat Routing-Scheduling: A BiObjective Stackelberg Game" submitted to Transportation Research Part E: Logistics and Transportation Review for publication consideration.

Overall, we appreciate the valuable comments of the reviewers, editor and EIC's comments, which helped us improve our paper substantially. Basically, we have adopted all the suggestions given by them and updated the results.

Attached are also our responses to the comments. We hope that the responses have answered their comments. We look forward to hearing from you soon again regarding the status of this revised paper. Thank you very much for taking care of this review process for us.

Yours sincerely,

Reza Zanjirani Farahani (Ph.D.)

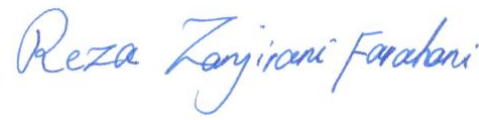

Yours sincerely,

Reza Zanjirani Farahani $(P h D)$

cc.

Mr. Seyed Sina Mohri $(M S c)$

Dr. Nasrin Asgari $(P h D)$

Prof. Michael Bourlakis $(P h D)$

Prof. Benjamin Laker $(P h D)$

\section{ACKNOWLEDGMENTS}

We would like to thank Dr. Szeto Wai Yuen from the University of Hong Kong and Prof. Gilbert Laporte for their valuable comments on the research. We also appreciate Dr. Justin Yates from Francis Marion University for sharing some of the references.

\section{Conflict of interest}

I have had co-authorship with Szeto Dr. Wai Yuen (Ph.D.) and Agachai Sumalee (Ph.D.) in the area. The EIC may need to know these facts when selecting the appropriate referees. There is also a conflict of interest with Prof. Gilbert Laporte. 